\title{
Clinical potential of the mTOR effectors S6K1, S6K2 and 4EBP1 in breast cancer
}

\author{
Elin Karlsson
}

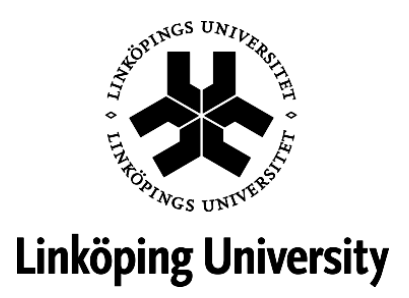

Division of Medical Sciences/Oncology

Department of Clinical and Experimental Medicine

Faculty of Health Sciences, Linköping University

SE-581 85 Linköping

Linköping 2014 
This study was supported by grants from the Swedish Cancer Foundation, the Swedish Research Council, King Gustaf V Jubilee Fund, the LiU Cancer Foundation, County Council of Östergötland, Borgholm Rotary Klubbs stipendiefond för onkologisk forskning, Lions forskningsfond mot folksjukdomar and Knut och Alice Wallenbergs jubileumsfond.

Main supervisor

Olle Stål

$\mathrm{PhD}$, professor experimental oncology

Division of Oncology, Department of Clinical and Experimental Medicine, Faculty of Health Sciences,

Linköping University

Co-supervisors

Anna-Lotta Hallbeck

$\mathrm{MD}, \mathrm{PhD}$, clinical oncologist

Clinical Oncology, CKOC, County Council of Östergötland, and Division of Oncology, Department of Clinical and Experimental Medicine, Faculty of Health Sciences, Linköping University

Patrik Lundström

$\mathrm{PhD}$, associate professor

Division of Molecular Biotechnology, Department of Physics, Chemistry and Biology, Linköping University

\section{(C) 2014 Elin Karlsson}

ISBN: 978-91-7519-432-5

ISSN 0345-0082

Published articles have been printed with permission of the copyright holders

Study 1 (c) Springer

Study 2 @ John Wiley \& Sons, Inc

Study 3 @ BioMed Central Ltd

Cover illustration: “The puzzle" photo Tomas Tollwé, Tranås, Sweden, 2013

Printed by LiU tryck, Linköping, Sweden, 2014 


\section{FÖRORD OCH FÖRFATTARENS TACK}

Bröstcancer är $i$ dag en av våra största folksjukdomar. Tack vare intensiv forskning och klinisk utveckling överlever nu allt fler denna sjukdom, men ännu behöver mycket mer göras. Jag är mycket tacksam över att ha fått möjlighet att delta $i$ denna kamp mot cancer under dessa år som denna avhandling sammanställts, och förhoppningen är att denna studie tillsammans med andra studier, kan vara en användbar pusselbit och ett steg $i$ rätt riktning.

Jag vill här rikta ett varmt tack till alla som gjort denna resa möjlig:

Drabbade patienter och anhöriga, klinisk och teknisk personal, samt bidragsgivare och alla som stödjer dessa arbeten runt om i vårt land. Patienters aktiva deltagande i kliniska studier och vävnadsdonation möjliggör forskningens fortsatta arbete och hjälper många.

Avd. för Onkologi vid Hälsouniversitetet, Linköping och dess ämnesföreträdare Charlotta Dabrosin och samordnare Chatarina Malm, för möjligheten att göra min forskarutbildning på avdelningen.

Olle Stål, min huvudhandledare under forskarutbildningen, som gav mig chansen att göra denna resa. Genom din stora ödmjukhet har du visat att det går att driva forskning "med hjärtat". Du är min förebild i forskningsvärlden.

Anna-Lotta Hallbeck, min bihandledare, som varit vår länk in till bröstcancerkliniken. Varje möte med dig är enormt lärorikt, och ger perspektiv.

Patrik Lundström, min bihandledare, som gett vårt projekt en ny vinkel med djupare biokemisk och bioteknisk kunskap. Tack för ett gott samarbete, inga projekt är omöjliga!

Alla nuvarande och tidigare vänner och kollegor på KEF, Cellbiologen och Valla; stort tack för alla goda samarbeten, för all hjälp och för att ni skapat en kreativ och hemtrevlig forskningsmiljö, lycka till med ert fortsatta arbete, med hopp om fortsatta samarbeten.

Alla examensarbetare och medförfattare som jag fått förmånen att jobba tillsammans med, det har varit väldigt givande och lärorikt.

Studenter och handledarkollegor i basgrupper och under alla labbar, er nyfikenhet har varit en stor inspirationskälla.

Tidigare lärare under alla år i skolan, som med er entusiasm väckt mitt intresse för naturvetenskap och biomedicin, däribland Biomedicinska forskarskolan 2006-2007.

Min familj och mina vänner som varit ett ovärderligt stöd under resan. Jag är övertygad om att omtanke om varandra, att vara ute i naturen och att musicera tillsammans är den bästa hjälpen för allt, inklusive att bearbeta forskningsproblem.

Elin Karlsson, Adelöv 2014 


\section{SAMMANFATTNING}

\section{Klinisk potential för cellernas nav mTOR och dess måltavlor S6K1, S6K2 och 4EBP1 för en bättre individanpassad behandling av bröstcancer}

Tumörsjukdomar, för kvinnor främst bröst-och lungcancer, utgör en av de främsta dödsorsakerna i västvärlden i dag. Tack vare utveckling av metoder för diagnos och behandling, liksom upptäckt på allt tidigare stadium har prognosen för bröstcancerpatienter förbättrats radikalt de senaste 25-30 åren. Dock diagnosticeras i Sverige ca 8000 nya fall av bröstcancer varje år, och omkring 1500 kvinnor dör av sjukdomen.

Ett stort problem är att brösttumörer är väldigt olikartade, och kanske egentligen borde betraktas som flera olika sjukdomar. Ett mål med dagens forskning är därför att hitta vägar för att kunna individanpassa behandling och därmed förbättra överlevnaden i bröstcancer, men också minska bieffekterna av onödiga behandlingar. En viktig del i detta är att öka kunskaperna om bröstcancerns biologi och förstå vad som egentligen händer i cancercellerna i olika former av tumörer. Därmed skulle man också kunna identifiera nya markörer i tumörerna som skulle kunna användas som ett tillägg till dagens ganska grova bedömningskriterier i kliniken.

Utveckling och tillväxt av den normala bröstkörteln från speciella stamceller, sker gradvis och i olika stadier av livet. Detta styrs genom ett komplext samspel mellan hormoner, främst östrogen och progesteron, samt olika tillväxtfaktorer mellan cellerna, och deras målmolekyler inne i cellerna. Man tror att brösttumörer uppkommer och tillväxer p.g.a. att detta samspel går överstyr och man får en överaktivering av tillväxtsignaler i körtelcellerna. En välkänd orsak är att cellerna får förhöjda nivåer av östrogenreceptorer, målmolekylerna för östrogen. I dag behandlas patienter vars tumörer har höga nivåer av östrogenreceptorer, med antiöstrogener.

På senare år har man identifierat faktorn mTOR, som en annan viktig målmolekyl och ett nav för integrering av olika tillväxtsignaler inne i normala celler, bl.a. från östrogen och insulin (se Figur nedan). Det har visats att människor som har medfödda mutationer som leder till att mTOR blir mindre aktivt i cellerna, i princip aldrig utvecklar cancer eller diabetes. Däremot leder detta till andra komplikationer, som dvärgväxt och annan underutveckling. mTOR är ofta överaktivt i tumörceller jämfört med normala celler. Sedan 2012 behandlas vissa patienter med spridd bröstcancer med en kombination av antiöstrogener och mTOR-hämmare. Långt ifrån alla svarar dock på denna behandling och mer kunskaper om mTOR och dess målmolekyler i cellerna behövs för att kunna optimera behandling och förstå när den gör nytta.

I denna studie har vi tittat på de tre mest kända av mTORs målmolekyler; S6K1 S6K2 och 4EBP1. Det var tidigare känt att S6K1 kunde finnas i höga nivåer i upp till $20 \%$ av alla brösttumörer och detta var associerat med sämre överlevnad hos dessa patienter. Nu har vi kunnat visa att även nivåerna av S6K2 är förhöjda i ungefär lika stor andel tumörer, dock sällan samtidigt som S6K1. Däremot verkar nivåerna av S6K2 och 4EBP1 ofta vara förhöjda i samma tumörer. Höga tumörnivåer av S6K2 och 4EBP1 samtidigt kunde kopplas till ökad risk att dö i bröstcancer. Vi kunde också visa att 
tumörnivåerna av S6K1, S6K2 och 4EBP1 kan användas för att förutsäga om en bröstcancerpatient bör ha nytta av antiöstrogenbehandling eller ej.

Vi har också tittat på möjligheten att utveckla nya, specifika behandlingar för att hämma mTORs målmolekyler mer specifikt. S6K1 och S6K2 är 70\% identiska till utseendet och har länge betraktats som biologiskt likvärdiga. Våra studier, tillsammans med andra nya studier, pekar dock på att S6K1 och S6K2 verkar kunna ha delvis olika roller, både i normala vävnader och i olika tumörtyper. I odlade cancerceller i provrör, har vi kunnat släcka ner aktiviteten av S6K1 och S6K2 individuellt och sett att detta verkar kunna ge olika effekter på nivåerna av andra faktorer i cellerna och även påverka celltillväxt. Genom att i detalj jämföra utseendet och strukturen på S6K1 och S6K2 har vi hittat mönster som skiljer sig åt så mycket att det borde vara möjligt att utforma specifika hämmare mot dessa faktorer.

Sammantaget tyder denna studie på att tumörnivåerna av S6K1, S6K2 och 4EBP1 skulle kunna fungera som biologiska markörer för att kunna bedöma bröstcancerpatientens prognos och förutsäga när antiöstrogen behandling är verksamt eller när ytterligare behandling behövs. Genom att utveckla specifika hämmare mot S6K1 respektive S6K2 skulle det vara möjligt att få en mer skräddarsydd behandling än med dagens mTOR-hämmare. Kunskap om mTORs målmolekyler S6K1, S6K2 och 4EBP1 skulle därmed kunna vara ett nytt verktyg för mer individanpassad diagnostik och behandling och därmed förbättrad bröstcanceröverlevnad i framtiden.

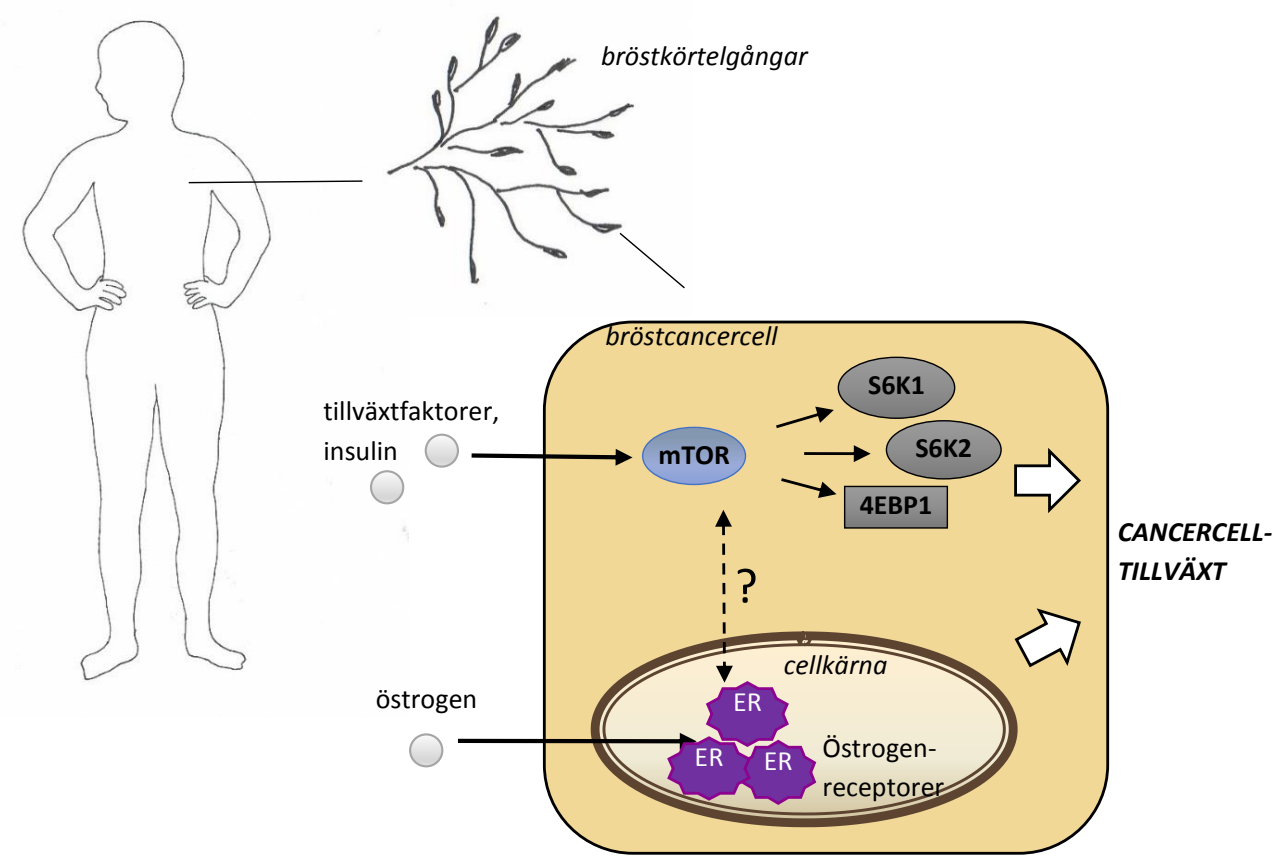

Figur Det komplexa samspelet mellan hormoner och olika tillväxtfaktorer som styr normal bröstkörtelutveckling går överstyr vid bröstcancer, vilket leder till okontrollerad celltillväxt. I cellerna tros mTOR och dess målmolekyler S6K1, S6K2 och 4EBP1 kunna utgöra ett nav i detta samspel. 


\section{TABLE OF CONTENTS}

FÖRORD OCH FÖRFATTARENS TACK ……............................................................................

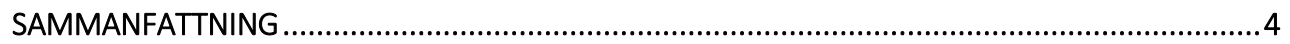

Klinisk potential för cellernas nav mTOR och dess måltavlor S6K1, S6K2 och 4EBP1 för en bättre

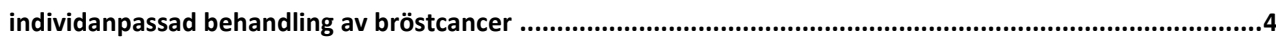

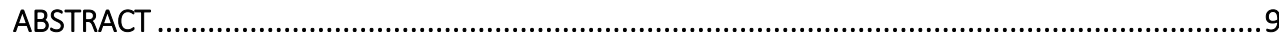

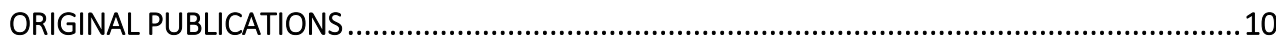

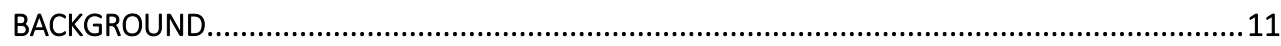

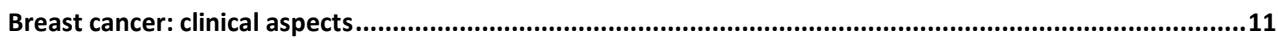

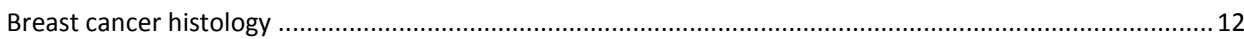

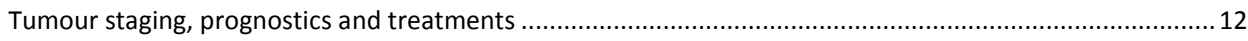

The need for further clinical markers of prognosis and prediction of treatment benefit ............................15

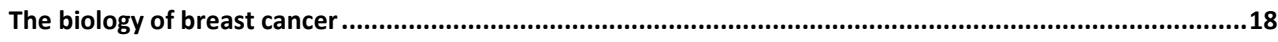

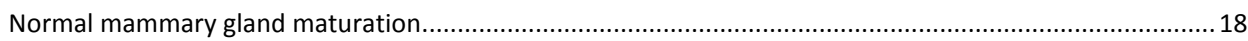

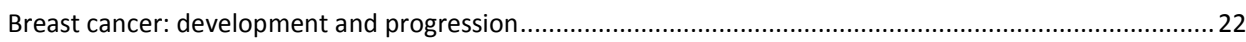

Importance of oestrogen and progesterone signalling in breast cancer ..................................................24

Features of tumourigenesis: the hallmarks of cancer .............................................................................. 25

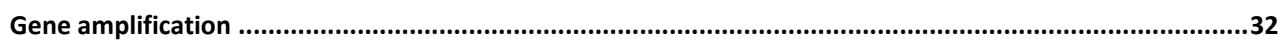

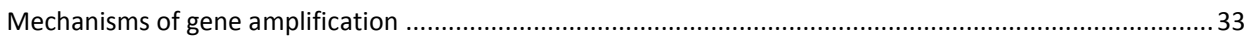

Amplicons as indicators of tumour driving oncogenes ............................................................................ 34

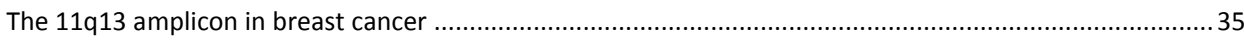

The mTOR/S6K/4EBP1 pathway in breast cancer..........................................................................................38

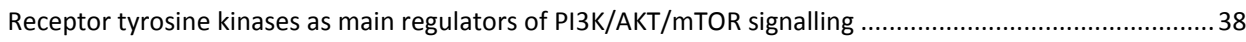

$\mathrm{PI3K} / \mathrm{AKT}$ signalling is the most overstimulated pathway in breast cancer ...............................................42

mTOR signalling from the two complexes mTORC1 and mTORC2 ….........................................................43

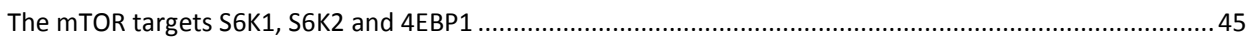

Activation of the PI3K/AKT/mTOR pathway as a clinical marker and target in cancer therapeutics ...............53

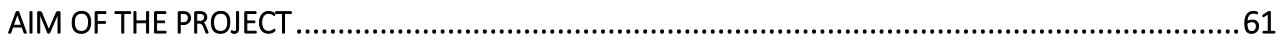

MATERIALS AND METHODS USED IN THE STUDIES ......................................................62

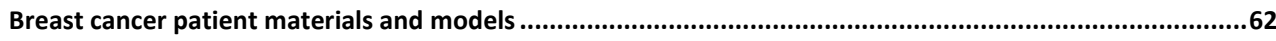

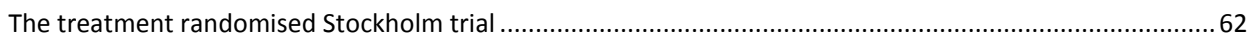




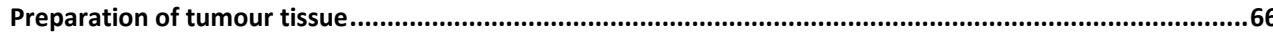

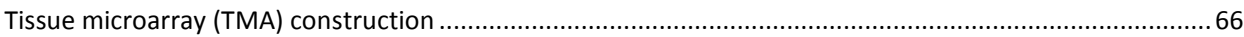

Extraction of genomic DNA and total RNA from fresh-frozen tissue .....................................................6 66

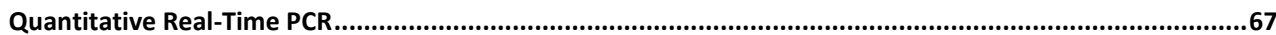

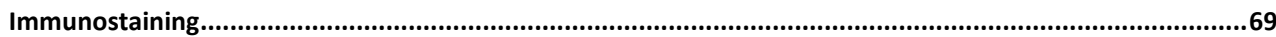

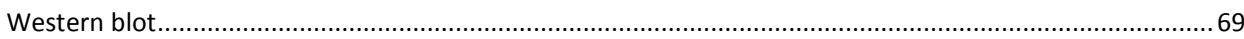

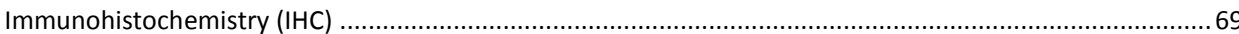

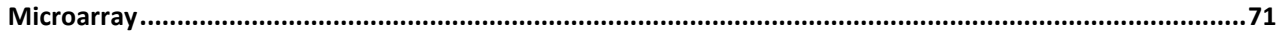

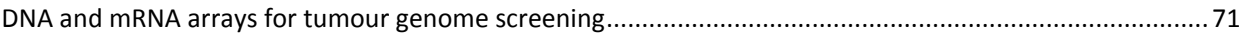

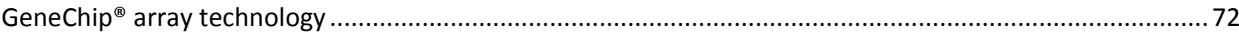

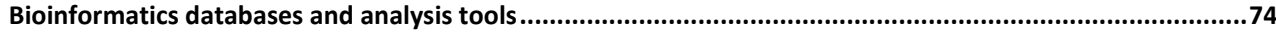

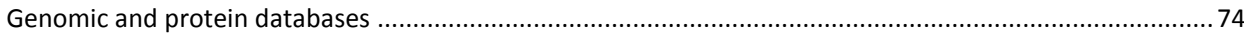

Tools for browsing genomic data, sequence alignments and PCR primer design ......................................74

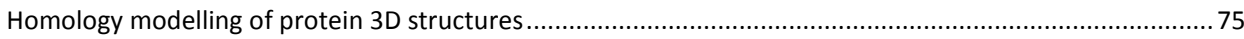

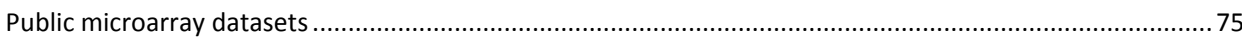

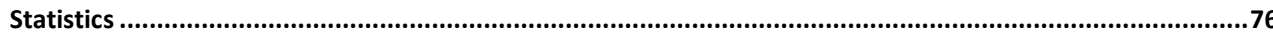

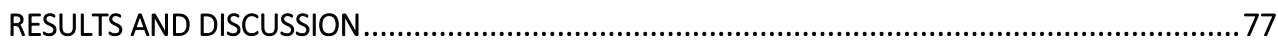

The mTOR effector S6K2 is a new candidate oncogene in the 11q13 amplicon in breast cancer ...................77

S6K2/4EBP1 coamplification and mRNA coexpression is associated with a poor outcome, indicating a synergy between mTOR targets

Whole-genome mRNA profiles of S6K2 and 4EBP1 positive tumours are highly overlapping, revealing associations with cell cycle regulators and IGF signalling

Intracellular localisation and expression levels of S6K1, S6K2 and 4EBP1 protein determine associations with clinicopathological factors and predictive role for endocrine treatment benefit

Structural comparison and in vitro silencing of S6K1 and S6K2 suggest a possibility to develop isoform specific inhibitors.

ABBREVIATIONS 


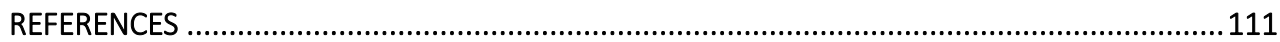


The prognosis of patients diagnosed with breast cancer has been considerably improved in the latest 25 years, as a result of continuous development of diagnostics and treatment regimens. Though, tumour diseases, for woman mainly lung cancer and breast cancer, still constitute one of the most common causes of death in developed countries, following heart diseases. A future utopia is to develop more individualised therapy strategies, to further increase breast cancer survival, but also to decrease the risk of severe side-effects of unnecessary treatments.

Normal mammary gland development is regulated by a complex interplay between growth factors and hormones, mainly oestrogen and progesterone, in different cell types. Breast cancer origin and progression is assumed to result from an imbalance in this interplay, leading to the so called "Hallmarks of cancer", including unlimited cellular proliferation. A central hub in the regulation of proliferation is the intracellular mTOR signalling pathway. Antioestrogen therapy is widely used in breast cancer clinics, however resistance towards this treatment is a remaining problem, and overactivation of mTOR may be one reason behind. A new treatment regimen constituting a combination of mTOR inhibitors with endocrine therapy was recently clinically approved for advanced breast cancers. Although significant benefit for this combination treatment is evident for some patients, counteracting feedback mechanisms are assumed to diminish the effects.

The work presented in this thesis focuses on the genes S6K1, S6K2 and 4EBP1 which are main effectors of the intracellular mTOR signalling pathway and thereby secondary targets of the mTOR inhibitors. Our results suggests that the gene amplification status, expression levels of the corresponding mRNA and protein of $\mathrm{S} 6 \mathrm{~K} 1, \mathrm{~S} 6 \mathrm{~K} 2$ and 4EBP1 as well as their cellular localisation may be used to predict breast cancer outcome and the benefit from antioestrogen treatments. These factors are indicated to play separate roles in different subtypes of breast cancer, and specific targeting of S6K1 and S6K2 may be valuable in different tumour subtypes, and in comparison to present day's mTOR inhibitors, further promote individualised therapies, and thereby increase breast cancer survival. 


\section{ORIGINAL PUBLICATIONS}

This thesis was based on the following original publications:

Study 1

Gizeh Pérez-Tenorio, Elin Karlsson, Marie Ahnström-Waltersson, Birgit Olsson, Birgitta Holmlund, Bo Nordenskjöld, Tommy Fornander, Lambert Skoog, and Olle Stål, Clinical potential of the mTOR targets S6K1 and S6K2 in breast cancer. Breast Cancer Res Treat, 2011. 128(3): p. 713-23. (GPT and EK contributed equally).

Study 2

Elin Karlsson, Marie Ahnström-Waltersson, Josefine Bostner, Gizeh Pérez-Tenorio, Birgit Olsson, Anna-Lotta Hallbeck, and Olle Stål, High-resolution genomic analysis of the 11q13 amplicon in breast cancers identifies synergy with 8p12 amplification, involving the mTOR targets S6K2 and 4EBP1. Genes Chromosomes Cancer, 2011. 50(10): p. 775-87.

Study 3

Elin Karlsson, Gizeh Pérez-Tenorio, Risul Amin, Josefine Bostner, Lambert Skoog, Tommy Fornander, Dennis C Sgroi, Bo Nordenskjöld, Anna-Lotta Hallbeck and Olle Stål; The mTOR effectors 4EBP1 and S6K2 are frequently coexpressed, and associated with a poor prognosis and endocrine resistance in breast cancer: a retrospective study including patients from the randomised Stockholm tamoxifen trials. Breast Cancer Res. 2013 Oct 17;15(5):R96

\section{Study 4}

Elin Karlsson, Ivana Magić, Josefine Bostner, Christine Dyrager, Fredrik Lysholm, AnnaLotta Hallbeck, Olle Stål and Patrik Lundström, Revealing potentially different roles of the mTOR-targets S6K1 and S6K2 in breast cancer by expression profiling and structural analysis. 2014, Manuscript. (OS and PL contributed equally). 


\section{BACKGROUND}

Breast cancer is despite advances in diagnostics and treatments still a leading cause of malignancy associated death among women worldwide. A high heterogeneity, encompassing a wide variety of pathological and clinical behaviours, as well as problems with identifying risk factors predisposing for breast cancer may explain the difficulties in controlling the disease. The future utopia would be further strategies for protecting against cancer development, for earlier detection by screening, and for a more individualised therapy, to increase breast cancer survival, and to decrease the risk of severe side-effects. For this purpose, additional tumour specific clinical markers and treatment targets are needed. Our understanding of breast cancer origin and progression can hopefully be improved by seeking to characterise the complex patterns of underlying genetic alterations and subsequent deregulations of signalling pathways.

The present study encompasses the mTOR signalling pathway, which is commonly deregulated in breast cancer and has recently emerged as a promising new therapeutic target. The focus was laid on the downstream effectors of mTOR; S6K1, S6K2 and 4EBP1, with an effort to evaluate their specific roles in carcinogenesis and to characterise their potential as clinical markers and as therapeutic targets. This introduction will give an overview of both the clinical and molecular aspects of breast cancer, concluding into what so far is known about the role of mTOR signalling within this context, and describe the specific aims of the present study.

\section{Breast cancer: clinical aspects}

The incidence of breast cancer in the western countries is increasing, a trend that is estimated to be real and not only a consequence of the aging of the population [1]. In Sweden, there are at present approximately 8000 new cases registered every year, with a median patient age at diagnosis of 65 years. Breast cancer mortality rates have yet remained relatively constant at 1500 deaths per year, even slowly decreasing, likely as a result of improved detection and treatment strategies [2].

In general, the aetiology of breast cancer remains unknown. Studies on inhabitants emigrating from low to high risk countries have indicated that the western life style and environment significantly increases the risk. Yet, apart from increased age, no specific factors in this sense have been pointed out. Hormonal features, in terms of early menarche, late menopause and late or few pregnancies have been suggested as predisposing, as well as diagnostic radiation against the mammary gland [1]. The most well-known risk factor is a family history of breast cancer, which is estimated to account for up to $10 \%$ of the cases. Germline mutations in the DNA-repair genes BRCA1 and BRCA2 are identified as responsible in a major part of cases, though other susceptibility genes are actively sought after (reviewed in [3]). At present, studies are evaluating the role of the TP53, STK11, PTEN, CDH1 and CHEK2 genes in familial breast cancer [4]. 


\section{Breast cancer histology}

The great majority of breast cancers are defined as carcinomas, arising in the epithelium of the lobules or the ducts of the mammary gland (for an overview of breast anatomy, see Figure 1). Initiation of malignancy is extremely rare in the stromal, vascular or fatty components of the breast [1]. To date, pathologists have defined 18 different histopathological subtypes of breast cancer [5]. Mainly, breast carcinomas are histologically classified as non-invasive (in situ) or invasive. Non-invasive lesions are further subdivided into lobular (LCIS) or ductal (DCIS) carcinoma in situ, based on the site of origin. LCIS does not form a palpable tumour and is not visible on mammography. This lesion is therefore a purely histological diagnosis, considered as a marker of increased risk for the development of invasive cancer. DCIS is defined as the precursor of invasive ductal carcinoma (IDC), which in turn is the most common form of breast cancer including $70-80 \%$ of all cases. Invasive lobular carcinoma (ILC) represents $5-15 \%$ of all breast cancers, whereas other rare, though specified invasive cancers can be exemplified by tubular, medullar, mucinous and papillary carcinomas.

\section{Tumour staging, prognostics and treatments}

The high heterogeneity of breast cancer proposes the importance of improved individualised prognostics and therapy strategies. Various prognostic and predictive factors are at present utilised, and additional markers are continuously evaluated. The staging and prognostic tools, as well as treatment strategies briefly covered in this section, are the mainly recommended in clinical routine by the Swedish Breast Cancer Group (Table 1) [6].

In general, the prognosis and treatment of a breast cancer patient is determined by the tumour stage at the time of diagnosis. The TNM classification system, stated by the Union for International Cancer Control (UICC), is widely used in clinical routine. Here, staging is based on the size of the primary tumour (T), axillary lymph node involvement $(\mathrm{N})$ and presence of metastases $(\mathrm{M})$. In addition to TNM classification, several other markers are used for assessing the risk of systemic recurrence and for determining adjuvant therapy. Histological grade describes tumour cell differentiation and is defined based on tubule formation, mitotic count and nuclear pleomorphism. Cell proliferation is commonly evaluated as the presence of Ki-67 antigen, evaluated with immunohistochemistry (IHC).

The primary treatment serves the purpose to achieve local control of the tumour. This mainly implies mastectomy or breast conserving surgery with complete local excision of the tumour tissue. Postoperative radiotherapy reduce the risk of local recurrence for all tumours, but the choice of this treatment is dependent on many factors, including possible side effects, the age and health condition of the patient. Radiotherapy is recommended following breast conserving surgery and in case of high grade tumours, as well as after resection of axillary lymph node metastasis. This commonly involves an absorbed dose of $50 \mathrm{~Gy}$, divided into 25 fractions, during 5 weeks.

Systemic adjuvant treatments are offered when there is risk of recurrent disease. Postoperative chemotherapy is commonly given in 4-6 cycles during 5 months after primary surgery. Today, this usually involves a combination treatment of 3 cycles fluorouracil/5FU, epirubicin and cyclophosphamide (FEC), followed by 3 cycles of taxanes. Steroid hormones, mainly oestrogen and progesterone, are known to play essential roles in breast cancer development and progression. The presence of oestrogen- (ER) and progesterone receptors (PgR), as evaluated by IHC, is the decisive 
factor for the choice of endocrine treatment. About $75 \%$ of breast tumours are diagnosed as ER positive, and since the late 1970s, the ER inhibitor tamoxifen has been the golden standard treatment for this group of patients. In recent years aromatase inhibitors, suppressing oestrogen synthesis, have been established as a second possible endocrine treatment for postmenopausal patients. Premenopausal woman may receive gonadotropin-releasing hormone $(\mathrm{GnRH})$ analogues as a complement to tamoxifen. In addition, fulvestrant which targets the ER for degradation, is a secondline treatment for postmenopausal woman with ER-positive disease, relapsing on previous endocrine therapies.

In the last decade, the human epidermal growth factor receptor 2 (HER2, ERBB2) has emerged as an important predictive factor, shown to be amplified and overexpressed in approximately $15 \%$ of breast cancers. In clinical routine, HER2 status is initially evaluated by IHC. Uncertain cases, as well as tumours classified as $2+$ or $3+$ (moderate or strong expression of HER2 protein) are further assessed for amplification of the corresponding gene, by in situ hybridisation (ISH). Patients whose tumours are judged as HER 2 positive by ISH are since 2006 offered treatment with the anti-HER2 antibody trastuzumab (Herceptin) in addition to chemotherapy.

In general, patients with $\mathrm{ER} / \mathrm{PgR}$ positive tumours with certain risk factors are offered endocrine treatment, whereas the choice of chemotherapy is based on the stage and aggressiveness of the tumour as determined by the factors mentioned above. Recurrence with distant metastases, generalised disease, is so far considered as incurable. In these cases, systemic treatments may still be able to restrain disease progression and stabilise the disease, equivalent to a chronic disease.

In addition to ER and HER2 inhibitors, several other targeted therapies have been, or are currently evaluated in clinical trials and a few are at present used in combination with chemotherapy regimens, for treatment of advanced breast cancer. These include bevacizumab (VEGF inhibitor), lapatinib (HER1 and HER2 inhibitor) and pertuzumab (prevent HER2 dimerisation). In 2012, the mTOR inhibitor everolimus was clinically approved in combination with the aromatase inhibitor exemestane, for the treatment of recurrent ER-positive breast cancer [7]. 
Table 1 Overview of the present clinically used prognostic and treatment predictive factors for breast cancer in Sweden (IHC: immunohistochemistry, ISH: in situ hybridisation)

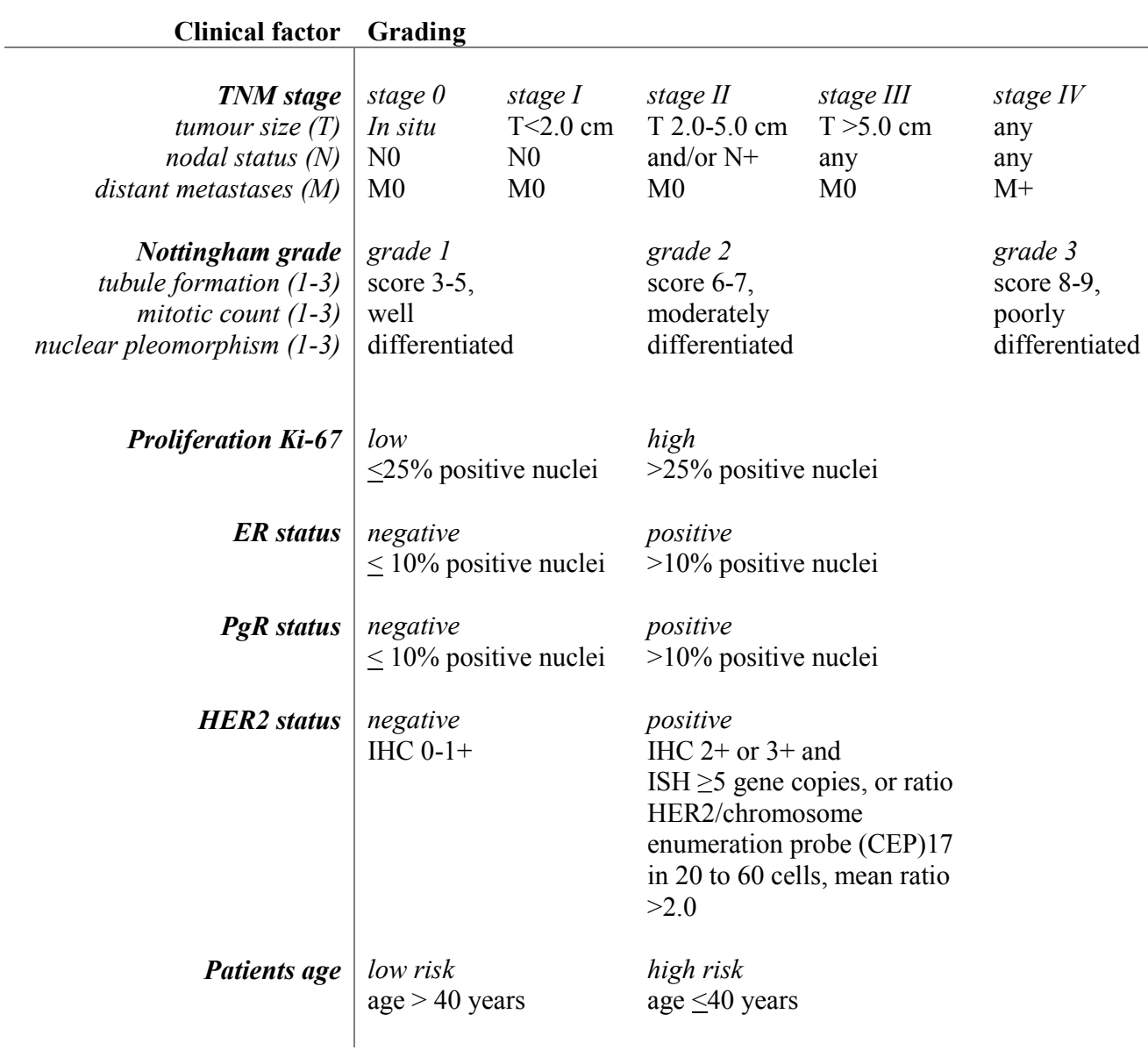




\title{
The need for further clinical markers of prognosis and prediction of treatment benefit
}

\begin{abstract}
Despite advances in prognostics and treatment, the present clinicopathological tools are still rather limited. Prognostic markers, indicating the natural progression of disease with or without treatment, as well as predictive markers of treatment benefit and new therapeutic targets for resistant tumours are highly needed. This would enable further patient specific therapies, lowering side-effects as a result of unnecessary treatments, as well as allow identification of patients in need for additional or prolonged therapies.
\end{abstract}

\section{New prognostic and treatment predictive markers evaluated in clinical trials}

Breast cancer is, as mentioned earlier, a highly heterogeneous disease, exhibiting a wide variety of genetic alterations, cellular features and clinical behaviours. Traditionally, breast cancers have been classified into prognostically meaningful groups at the clinical and histological level, however, it is increasingly evident that cellular and molecular features are of equal importance [8]. At present, several new indicators and markers for prognosis and treatment prediction are evaluated in clinical trials.

In addition to age, other physiological factors such as body mass index (BMI) and physical activity are suggested as valuable factors in prognostics. Regarding tumour specific markers, presence of angiogenesis, as well as high proliferation are indicators of poor prognosis. In addition to Ki-67 and mitotic index, the expression of proliferation cell nuclear antigen (PCNA), as well as cyclin A and phosphohistone $\mathrm{H3}$ (PPH3) are evaluated as new proliferative markers [6]. The urokinase plasminogene activator (uPA) and its inhibitor plasminogen activator inhibitor type 1 (PAI-1) are potential predictive markers for tumour invasion.

Even in the case of well-established clinical markers, essential issues are the significance of thresholds for positivity, as well as differential expression in different parts of the tumours and even more between primary tumour and metastasis. At present, Swedish recommendations for the definition of ER positivity is $>10 \%$ positive nuclei [6]. Some studies, though, have shown a benefit from endocrine treatment for patients with tumours harbouring $<10 \%$ ER-positive cells, therefore St. Gallen and American Society of Clinical Oncology (ASCO) guidelines since 2009 recommend $>1 \%$ ER positivity as a standard. There are also ongoing discussions proposing that ER status should not be stated in discrete values, rather on a continuous scale (oral presentation, Åke Borg, Villa Aske 2013). The predictive role of PgR expression has been debated in the light of contradictive studies, possibly resulting from methodological problems. Recently, the St. Gallen and ASCO guidelines stated that PgR has predictive value in addition to ER, for endocrine treatment benefit $[9,10]$.

In recent years, it has been shown that the expression of common prognostic and predictive markers alters between primary tumour and metastasis in a significant number of cases, and re-evaluation of ER, PgR and HER2 status in biopsies from metastases are now recommended when possible $[6,11]$. 


\section{Treatment resistance, with focus on endocrine therapies}

A main issue in breast cancer clinics is the occurrence of de novo or acquired treatment resistance. Underlying mechanisms of resistance as well as predictive markers of treatment benefit are actively sought. Antracyclin-based chemotherapy acts by blocking replication through intercalation with DNA and inhibition of topoisomerase IIa, and in turn topoisomerase IIa expression is suggested as a marker of benefit from this therapy. The presence of p53 mutations has been related to less response to antracyclins, and good benefit from taxanes, disrupting microtubule formation [6]. To monitor the effect of treatments on metastatic breast cancer, a new method for detection of circulating tumour cells (CTCs) in serum and plasma was recently approved [6].

For ER positive breast cancers, tamoxifen significantly reduces the recurrence and death rates among these patients. In the mammary gland, tamoxifen acts as an anti-oestrogen by competitively inhibiting the ER. In other tissues, the drug can also act as an agonist of the ER, and has been shown to reduce cholesterol levels and prevent coronary heart diseases. Even if most ER positive tumours respond well to tamoxifen, still about $30 \%$ of tumours remain resistant to endocrine therapy, either de novo or acquired during the treatment. Cross-talk between ER and growth factors signalling pathways, mainly the receptor tyrosine kinase (RTK)/PI3K/AKT/mTOR axis, is suggested as a mechanism of endocrine resistance $[12,13]$. As mentioned above, mTOR inhibitors in combination with endocrine therapies are promising, indicating the importance of this pathway in this context. Other suggested mechanisms of endocrine resistance include loss of ER expression or expression of truncated isoforms, posttranslational modification of the ER, deregulations of cofactors or the downstream $\mathrm{CDK} 4 / \mathrm{CDK} 6 / \mathrm{pRb} / \mathrm{E} 2 \mathrm{~F}$ cell cycle axis, as well as changes in tamoxifen metabolism, which may also result from overstimulation of growth signalling pathways $[12,13]$.

\section{Whole genome breast cancer classification}

In recent years, promising trials have been initiated in portraying tumours on a genome wide scale. A breakthrough in breast cancer classification was done by researchers at the Norwegian Radium Hospital and Stanford University, who revealed five subtypes of breast cancer based on their gene expression profiles [14]. These five subtypes, referred to as luminal A, luminal B, HER2+, basal-like and normal breast-like, have been shown to be conserved across ethnical groups and are already evident at pre-cancerous stages [15]. This apparent internal homogeneity, together with the heterogeneity between tumours indicate specific tumour progression pathways for each tumour subtype. One hypothesis is that the different subtypes originate from different types of stem or progenitor cells [15]. The subdivision of tumours into these groups has also been shown relevant from a prognostic point of view [16].

Based on genome-wide gene expression data, several multigene assays have been developed and shown able to predict outcome in certain patient groups [17]. The Real-Time polymerase chain reaction (PCR) based Oncotype Dx profile involving 21 genes, can be used to predict overall survival among patients with node-negative ER-positive breast cancers treated with tamoxifen. The MammaPrint signature, encompassing 70 genes derived from expression arrays, is a useful prognostic indicator in the ER positive as well as the ER negative subgroup. A similar profile, encompassing 50 genes constitute the PAM50 gene array, which is mainly used to detect high risk patients among ER 
positive, tamoxifen treated patients. The ratio of the two ER-regulated genes HoxB13 and IL17BR has also been used in the $\mathrm{H} / \mathrm{I}$ index to predict prognosis in endocrine treated as well as untreated patients with ER positive disease.

In a large and comprehensive study, Wood and colleagues have given their view of breast- and colorectal cancers based on genome wide mutation profiles [18]. When sequencing all, known to date, protein coding genes in eleven breast- and eleven colorectal cancers, approximately 80 point mutations leading to amino acid exchanges were found in each tumour. Overall, the sites of mutations appear to be very infrequent, with only a few commonly mutated genes. Statistical analyses have indicated that in each tumour, less than 15 of the mutations are responsible for driving tumourigenesis, whereas the majority of alterations are harmless and a result of genomic instability. A large challenge is therefore to distinguish tumour "drivers" from "passengers" in that sense. The number of potential driver genes is large, however, it is suggested that all changes together actually reflect alterations in a limited number of signalling pathways. This is in agreement with the fact that different genotypes can result in the same phenotype [18]. Thus, targeting pathways rather than genes may be an important clinical consideration.

Another aspect regarding genomic classification of tumours is alterations in DNA copy number, which as with the transcriptome, now is possible to study on a whole-genome scale. It is indicated that the overall pattern of gene amplifications is quite concordant with elevated gene expression and further studies are ongoing with the aim to investigate the global impact of DNA copy number changes on the five gene expression patterns in breast tumours [16]. Indeed, certain patterns of DNA copy number alterations have been associated with the different expression profiles [19]. Comparisons between histological phenotypes and the patterns of genetic alterations have revealed new insight in breast cancer progression [8]. The molecular profiles differ significantly between tumours with different histological grade, suggesting that low grade carcinoma rarely is a precursor of high grade and that this classification rather reflects diverse ways of tumour progression. On the other hand, no evident associations between histological types and specific patterns of genetic alterations have been elucidated, raising the question whether these designations actually are appropriate [8].

Comprehensive studies of breast cancer on a molecular level have significantly increased our understanding of the disease. However, an integration of the clinical, histological, cellular and molecular definitions of breast cancer is certainly needed for a better management of the disease and for finally being able to translate all findings into clinical practice. An improved understanding of the biology of breast cancer would facilitate this integration. 


\section{The biology of breast cancer}

Tumourigenesis is proposed to be a multistep process, with an underlying mechanism analogue to Darwinian evolution. Through stepwise genetic and epigenetic changes, progenitor cells and stromal cells receive defects in the systems controlling normal cell proliferation and homeostasis. This promotes selection of characteristics, by Hanahan and Weinberg referred to as the "Hallmarks of cancer" $[20,21]$.

Breast cancer is a highly heterogeneous disease, at the genetic as well as the clinicopathological level. However, based on gene expression profiling, five main tumour subtypes have been distinguished [14]. These subtypes were suggested to be derived from different stages in the normal mammary gland development, from gland stem cells to differentiated myoepithelial and luminal cells [22, 23]. These findings support the hypothesis of malignancy arising in certain cancer stem cells or progenitor cells and propose a new dimension on the view of breast tumour development, as cells on certain stages may give rise to different subtypes of breast cancer. This section will summarise the current achievements in the efforts to characterise the biology of breast cancer, starting with the cellular mechanisms regulating normal gland development.

\section{Normal mammary gland maturation}

The mammary gland is a highly complex organ, with the ability to undergo dynamic morphological changes during development and maintain the capability of remodelling and regeneration in a cyclical fashion during pregnancy [24]. The ductal tree is embedded within the fat pad, constituting a complex stroma including adipocytes, fibroblasts, immune cells, blood vessels and nerve cells (Figure 1 a). The mammary duct consists of a hollow lumen surrounded by an inner layer of luminal epithelial cells, a middle layer of myoepithelial cells and an outer basement membrane (Figure $1 \mathbf{b}$ ). The ability of a constant remodelling process requires the presence of a population of mammary stem cells constitutively able to differentiate into mature myoepithelial and luminal cells. The number and regenerative abilities of mammary stem cells are tightly regulated by oestrogen and progesterone levels, although the cells do not express ER or PgR. It is estimated that ER and PgR are expressed in $30-50 \%$ of luminal epithelial cells, though not in myoepithelial or basal cells, in the normal mammary gland. The ER+/PgR+ population of cells is non-proliferative, yet stimulates proliferation of ER/PgR- cells, including the clone of mammary gland stem cells, through paracrine signalling. The balance between ER+/PgR+ and ER-/PgR- cells is suggested to be partly regulated by the levels of $\mathrm{TGF} \beta 1$ and the transcription factor $\mathrm{C} / \mathrm{EBP} \beta$, and the number of $\mathrm{ER}+/ \mathrm{PgR}+$ cells are increasing with age, simultaneously with an increased breast cancer risk. 
a)

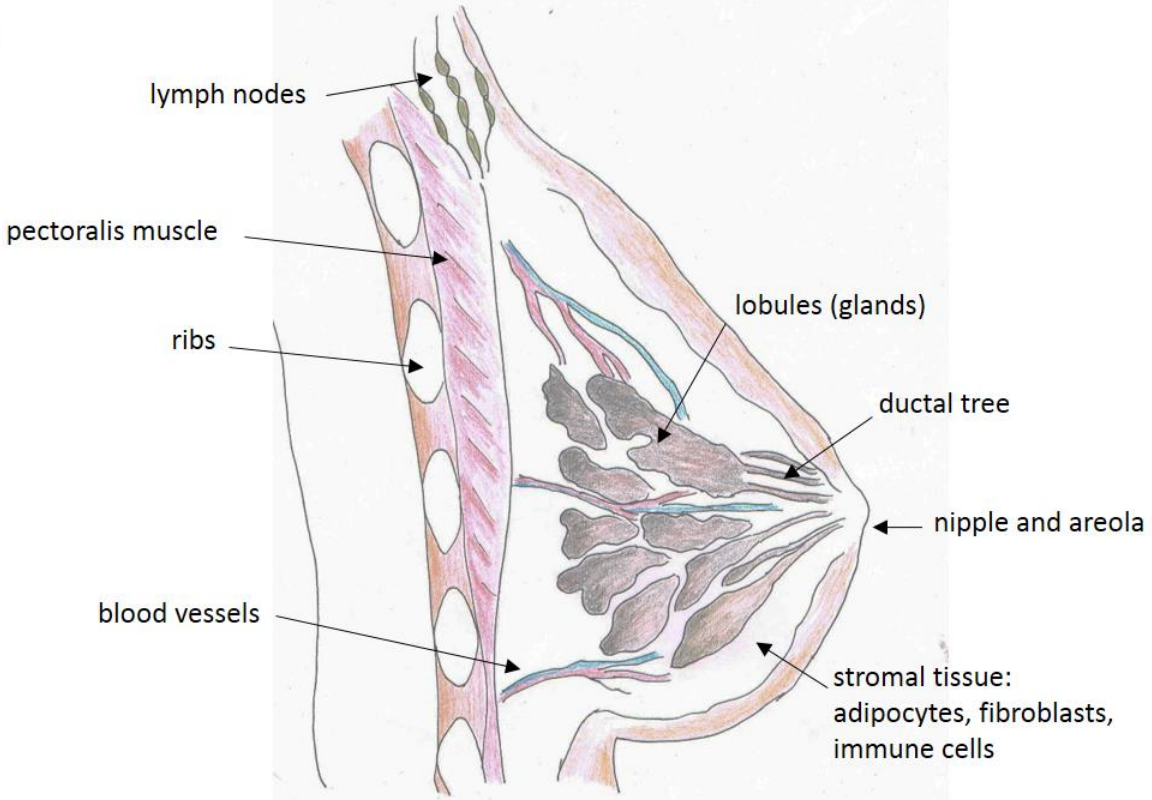

b)

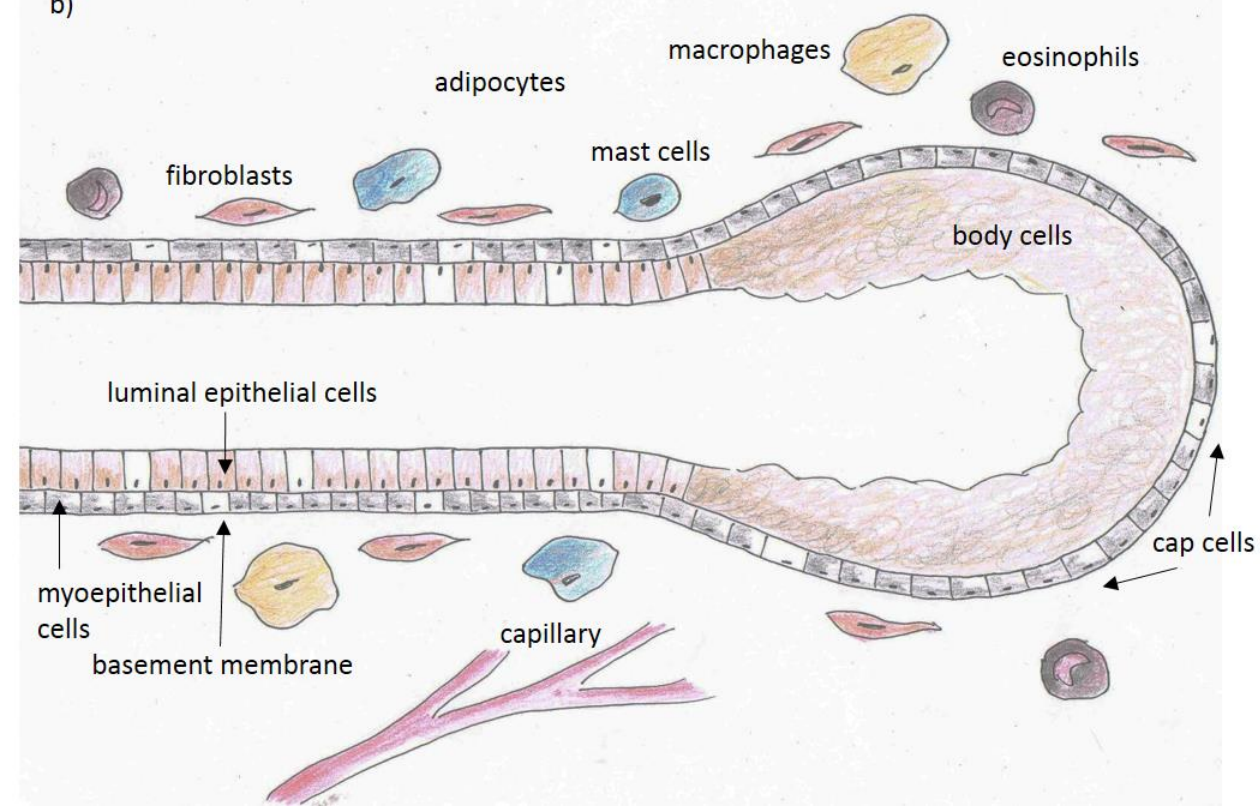

Figure 1 Mammary gland anatomy (a) and cells of the ducts and lobules [24] (b). 
Briefly, the first step of mammary gland development occurs during mid-gestation with the formation of five pairs of placodes in the epithelial cell layer, which invaginate into the mesenchyme leading to formation of mammary buds. Through proliferation steps, the mammary buds extend into 10-20 sprouts. This rudimentary ductal structure has formed by embryonic day 18.5, and remains quiescent until puberty when increasing levels of hormones and growth factors promote proliferation of the ductal ends into terminal end buds (Figure 2 a). These in turn continue to develop into an epithelial branching structure, eventually filling the space of the fat pad. During the cycles of pregnancy, the luminal epithelium proliferates and differentiates into secretory alveoli, producing milk into the lumen. At the end of lactation, involution of the secretory units occurs, involving removal of $80 \%$ of the epithelium through an apoptotic process.

The development and remodelling steps are orchestrated through a tightly regulated and time coordinated process requiring signalling between the several cell types and stromal components. The process is initiated by endocrine signalling from the ovaries and pituitary, mainly involving oestrogen, progesterone and growth hormone (somatotrophin), respectively. These hormones signal to both epithelial and stromal cells (Figure 2 b). Growth hormone binds to its receptors in stromal cells, leading to production of IGF1, in turn binding IGF1R at the epithelial cells, promoting their proliferation.

Oestrogen signals through ER in both stromal and epithelial cells, stimulating production of HGF and AREG, respectively. HGF stimulates epithelial proliferation by binding to the HGFR, while AREG binds the EGFR on the stromal cells. Progesterone signals through PgRs on the epithelial cells, stimulating expression of mainly AREG and RANKL, stimulating proliferation of epithelial cells in a paracrine manner. Stromal cells also produce various other growth factors e.g. TGF $\alpha$ and FGFs and the epithelial cells respond to additional receptors including EPHA2, FGFR1 and HER2/ERBB2. In addition, matrix metalloprotaeases (MMPs) secreted in a tightly controlled pattern during gland development, plays important roles by degrading of ECM, as well as in controlling cell migration and survival (Figure 2 b).

As will be shown below, many of the factors essential for normal gland maturation are deregulated during malignant transformation and are suggested as drivers of tumourigenesis and therefore potential treatment targets. 
a)

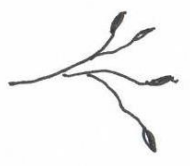

birth
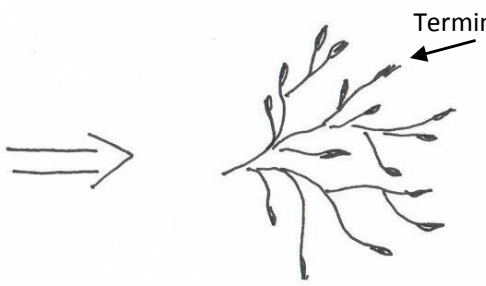

Terminal end buds

puberty

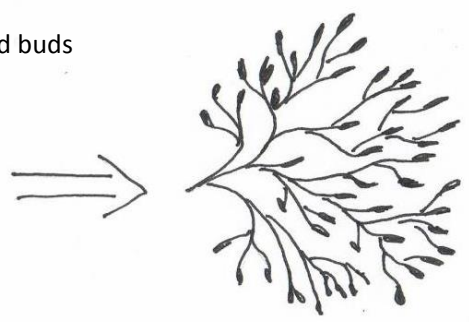

pregnancy

involution

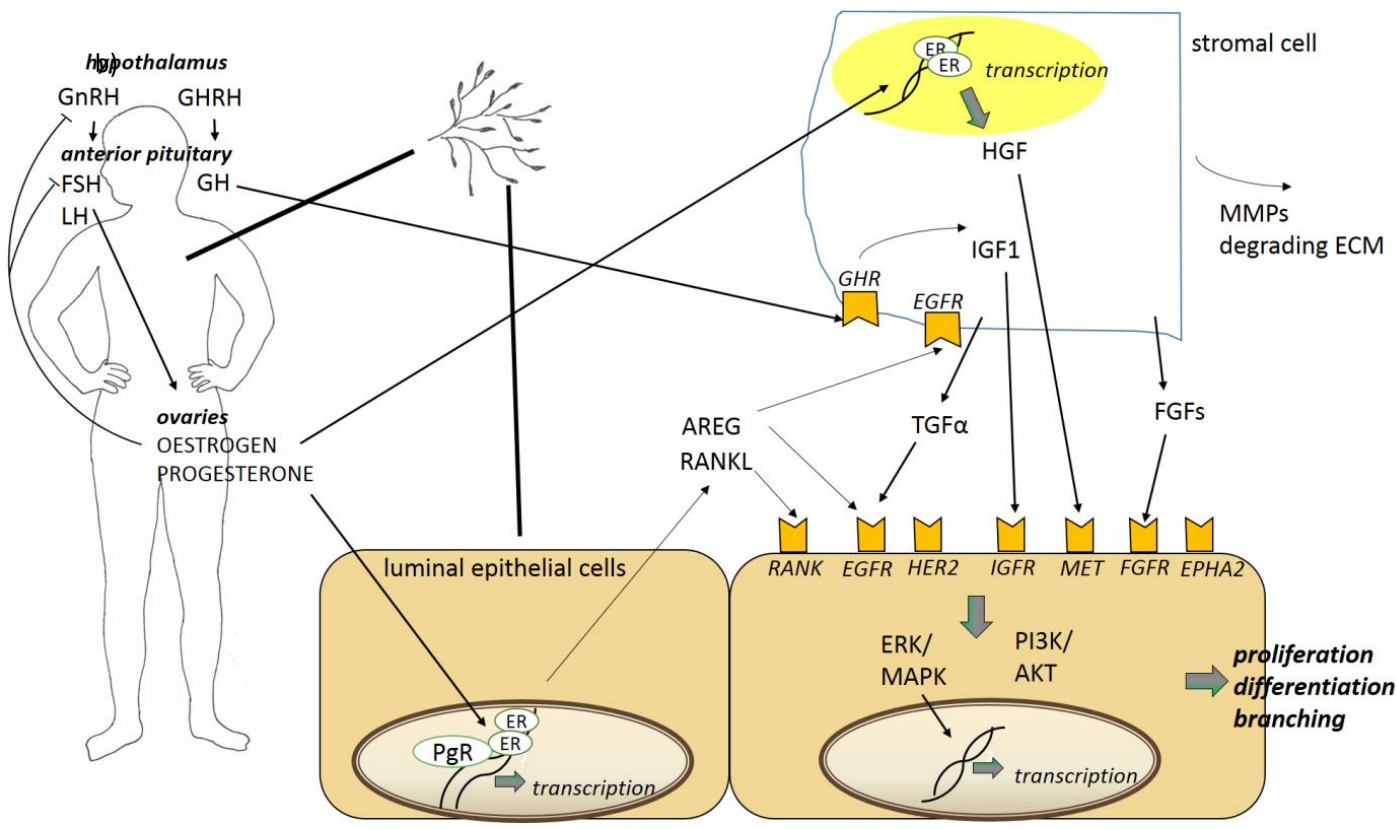

Figure 2 Mammary gland development; developmental stages of the ductal tree (a), endocrine and paracrine signalling regulates mammary gland differentiation (b). (GnRH: gonadotrophin-releasing hormone, FSH: follicle-stimulating hormone, LH: luteinising hormone; GH: growth hormone, GHRH: GH releasing hormone, ECM: extra-cellular matrix). 


\section{Breast cancer: development and progression}

Cancer development and progression is considered a multistep process, driven by epigenetic and genetic changes, as well as aberrant interactions within the microenvironment (reviewed in [15]). According to the clonal evolution theory, the transition of normal breast epithelial cells to a premalignant state involves genetic and subsequent phenotypic alterations in the myoepithelial cell layer [15]. Concurrently, the number of stromal cells increases. The carcinoma becomes invasive as a result of loss of myoepithelial cells and a subsequent degradation of the basement membrane. At this stage, cells are prone to invade surrounding tissues, enter the vasculature and migrate to distant organs, eventually giving rise to metastases. The whole tumour entity usually consists of both invasive and in situ components. Pre-invasive lesions are suggested to be quite common among the whole population, nevertheless, the mechanisms protecting some people from the transition to an invasive carcinoma remain unknown [15].

In recent years, a theory of cancer arising in certain types of stem cells has emerged. This so called cancer stem cell (CSC) theory has been intensively debated, in relation to the clonal evolution theory [25]. Proponents argue that for cancer cells to acquire the wide range of hallmarks as described below, the ability of the high plasticity of stem cells is needed. The high heterogeneity of tumours may also be explained by the CSC theory. Breast CSCs are suggested to originate from normal mammary gland stem cells, or from malignant epithelial cells going through epithelial to mesenchymal transition (EMT) [26]. To date, no available methods can with certainty distinguish normal stem cells from CSCs, obstructing the proving of their existence. CSCs are at present defined by the expression of certain stem cell markers, mainly CD44, CD24 and ALDH1, their properties of forming mammospheres in vitro, and the ability of a few CSCs to form a tumour after xenograft transplantation. Whether the phenotypic markers of CSCs also are functional remains to be investigated. Breast cancer originating from stem cells may be more aggressive than tumours arising from differentiated cells, and a high number of CSCs in tumours are connected to a poor outcome. However, a tumour may contain several different types of cells, all possessing stem cell characteristics, and identifying the cell-of-origin for a tumour is difficult. It has been suggested that CSC characteristics may result from mutations in specific signalling pathways.

Overall, it is likely that cancer development and progression may be best explained by a combination of the above theories, where stem cell characteristics facilitate clonal evolution leading to the hallmarks of cancer. As mentioned above, recent data propose that different molecular subtypes of breast cancer may arise from cells in different steps of the normal mammary gland development. According to this, differentiated luminal or myoepithelial cells may give rise to luminal, hormone responsive breast cancers, whereas mammary stem cells are the source of claudin-low and basal-like subtypes, associated with a more mesenchymal expression signature and more aggressive tumours [22, 23], (Figure 3). 


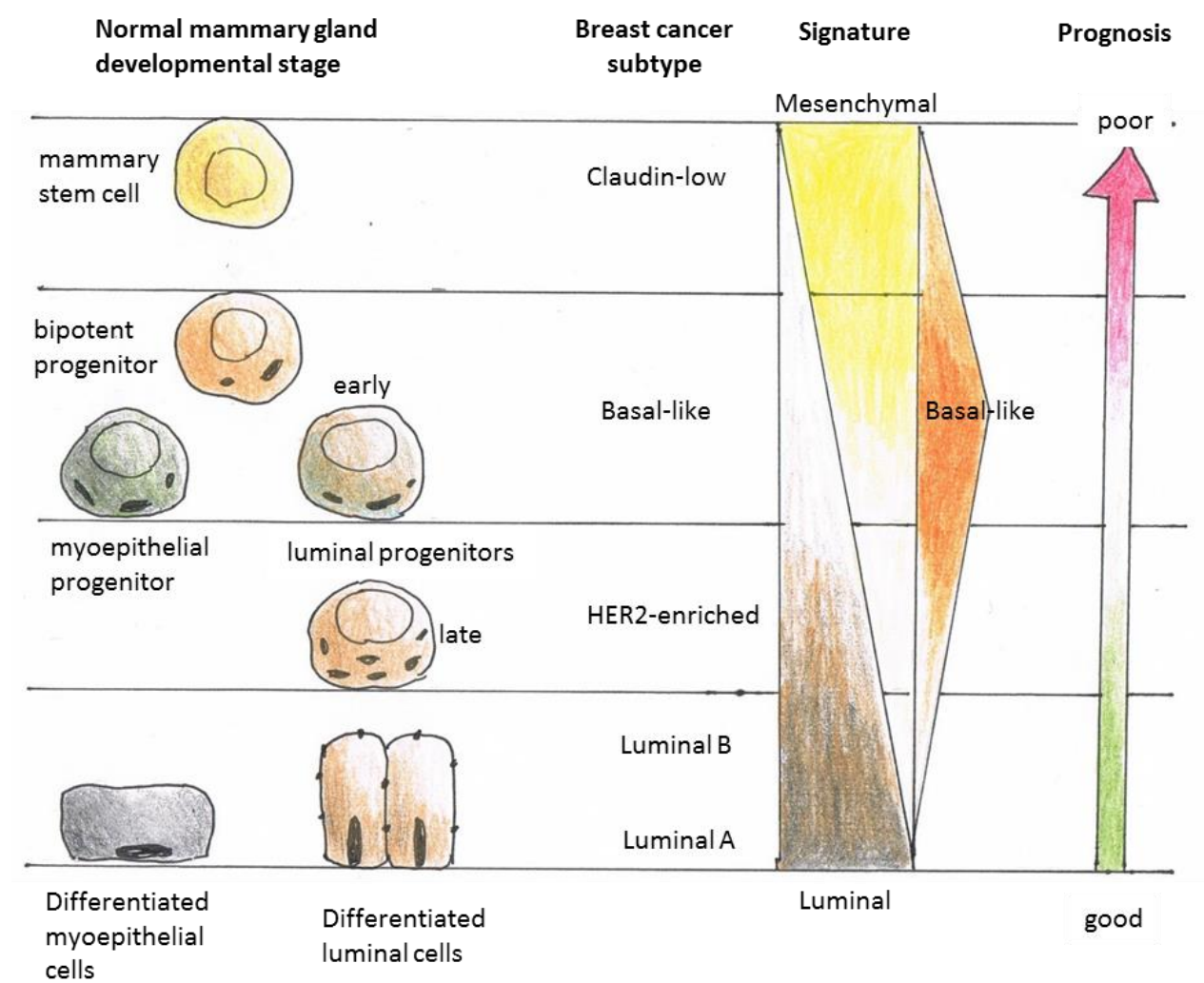

Figure 3 Subpopulations of cells in normal breast tissue are potential cells of origin for the different subtypes of breast cancer. 


\section{Importance of oestrogen and progesterone signalling in breast cancer}

Steroid hormones have long been known to play important roles in the development and progression of breast cancer. This was first shown by George Beatson, who in 1896 published the results of an improved breast cancer outcome and decrease in tumour size for premenopausal patients after surgical removal of the ovaries [27]. Subsequent studies revealed that not all patients had benefit from ovarian removal, and no method to predict response to the treatment was able until the discovery of the ER in the 1960s $[28,29]$. It is now known that about $75 \%$ of breast cancer tumours exhibit ERs, and in these cases oestrogen facilitates growth of the tumour. Oestrogen and progesterone are synthesised from cholesterol in several enzymatic steps. Oestrogen exists in many shapes, where oestradiol (E2) is the most biological active form in breast tissue. Premenopausally, the ovaries are the main oestrogen producers, whereas circulating androgens secreted from the adrenal gland are the primary oestrogen source after menopause. In general, oestrogen diffuses through the plasma membrane of the cell and binds to ERs in the nucleus, though also oestrogen action via membrane bound ERs are observed. ER $\alpha$, encoded by ESR1 at $6 \mathrm{q} 25$, is the classical prognostic and predictive marker in breast cancer, whereas the importance of the second ER isoform ER $\beta$, encoded by ESR2 at 14q23, remains unclear. The ER-oestrogen complex mediates its biological function by increasing or decreasing the transcriptional activity of certain genes, foremost involved in cell cycle regulation, thus promoting proliferation [30]. In the classical mechanism, E2 binds nuclear ER, causing a conformational change in the receptor, promoting posttranscriptional modifications, coactivator recruiting and receptor dimerisation. Subsequently, the ER-complex binds oestrogen-responsive elements (EREs) in the promoter region of its targets genes, including among others the $M Y C$ gene. Approximately $1 / 3$ of ER targeted genes are regulated through the non-classical mechanism, where ER affects transcription indirectly, by binding other transcription factors, e.g., the AP1 complex including $\mathrm{C}$-fos/C-jun. These genes involve e.g. CCND1, IGF1 and SERPINB. ER may also directly bind ERE-halfsites or binding sites of SP-1, as is the case in regulation of the $P R$ and also reported for CCND1.

$\mathrm{PgR}$, encoded by the $P R$ gene at 11q13 exists in two isoforms, PgRA and PgRB, where PgRA has a shorter amino terminal domain [31]. In vitro, PgRB is a stronger transcriptional activator than PgRA. The two isoforms are partly differentially expressed, e.g. PgRA is mainly expressed in the ovaries and uterine whereas PgRB is foremost involved in development and proliferation of the mammary gland epithelium. Like ER, the $\mathrm{PgR}$ is a transcription factor acting through cis-acting progesterone responsive elements (PREs) or through binding to other transcription factors. PgR regulated genes involve among others RANKL. The PgR also has non-transcriptional roles in the cytoplasm, activating phosphorylation cascades within minutes in vitro. The activity of $\operatorname{PgR}$ is regulated by ligand binding, as well as phosphorylation by among others MAPK, CDK2/cyclin A and CK2. In general, sustained high levels of oestrogen and progesterone are predisposing for breast cancer, but as clinical markers ER and PgR are associated with low grade tumours, a favourable short-term prognosis and response to endocrine therapies [32]. Alterations in the balance between PgRA and PgRB have been observed in tumour cells, and a high PgRA/PgRB has been associated with poor prognosis and less response to endocrine therapies [31]. In the majority of neoplasms, the population of proliferating $\mathrm{ER}+/ \mathrm{PgR}+$ luminal epithelial cells is increasing, likely as a result of a switch from paracrine to autocrine steroid hormone signalling through RANKL. Cyclin D1 is required for the proliferation of $\mathrm{ER}+/ \mathrm{PgR}+$ cells, and in line with this cyclin D1 is commonly amplified and overexpressed in ER/PgR + breast tumours [32]. Paracrine signalling may be more important in ER/PgR- breast cancer, and progesterone has been shown to stimulate proliferation of ER-/PgR- cells only in the presence of RANKL [31] 


\section{Features of tumourigenesis: the hallmarks of cancer}

In a highly attentive article in 2000, Hanahan and Weinberg proposed that the wide range of genetic alterations found in malignant cells often result in a few essential capabilities common for most tumours [20]. This global view of cancer has been very well accepted among the majority of cancer researchers, and recently an updated and refined description of the so called "hallmarks of cancer" was presented [21]. This section will briefly cover each of these basic capabilities and strategies by which it can be acquired in human cancers in general, and breast cancer in particular (Figure 4).

\section{The hallmarks of cancer}

$>$ Overactivation of growth signalling

$>$ Deregulation of antigrowth signals

$>$ Apoptosis resistance

$>$ Metabolic reprogramming

$>$ Limitless replication

$>$ Angiogenesis

$>$ Metastasis

$>$ Evading immune destruction

Figure 4 The proposed hallmarks of cancer [20, 21].

- Genomic instability

- Tumour microenvironment

- Chronic inflammation

\section{Activation of growth signalling}

All normal cells are dependent on mitogenic stimulatory signals for their ability to grow and proliferate. These signals are mediated by diffusible growth factors binding to transmembrane receptors which transmit the signals into the cells, and further to intracellular circuits, finally translating the signals into action (Figure 5). Growth is also stimulated by the interaction between cell adhesion molecules and extracellular matrix (ECM) components. During tumour development, cells acquire the capability to generate these signalling cascades more or less independent of exogenous growth stimulation [20,21]. The release of mitogenic signals are often increased in cancer tissue and may be mediated through autocrine signalling by the cancerous cells, or through malignant cells stimulating stromal cells to release growth factors in a paracrine manner. As previously mentioned, oestrogen signalling is a main determinant of cell proliferation in breast cancer, mediating a proliferative effect primarily by increasing the transcriptional activity of cell cycle promoting genes [33], and also through cross-talk to several growth signalling pathways [34] (Figure 5). Upregulation of steroid converting enzymes leading to increased local production of oestrogen in breast cancer tissue is also reported [35].

Intracellular growth signalling pathways are shown to be deregulated in most tumours. The PI3K/AKT pathway [36] regulates several cellular processes, including growth and proliferation, after activation by RTKs (Figure 5). Another intensively studied pathway is the Ras/MAP kinase pathway (Figure 5), which is frequently overactivated in many cancers. The growth signalling autonomy of cancer cells appears even more complicated, when considering that several intracellular pathways are 
cross-connected in a complex circuit, which is far from unravelled, enabling extracellular signals to mediate multiple effects (Figure 5).

\section{Deregulation of antigrowth signals}

To maintain tissue homeostasis in normal cells, proliferative and antiproliferative signals counterbalance each other in synergy. Antigrowth signals act mainly through the Gap1 (G1)checkpoint of the cell cycle (Figure 5). During the G1-phase, the cell monitors the internal and external environment to ensure that conditions are suitable before entering the DNA-replicative synthesis (S)-phase. Antigrowth signals can block proliferation by forcing the cell into a resting Gap0 (G0)-state or a postmitotic state. This is mainly mediated through TGF $\beta$, which prevents phosphorylation of the retinoblastoma protein $(\mathrm{pRb})$ through activation of p21 and p27 (Figure 5). Unphosphorylated $\mathrm{pRb}$ blocks proliferation by inactivation of the E2F transcription factor that controls expression of genes involved in G1 to S-phase progression. Myc is a transcription factor that among many other functions has been shown to regulate the G1 cell cycle checkpoint. In normal cells, stimulation of E2F1 and Myc induces a negative feedback on proliferation by inducing expression p53 (Figure 5). The signalling circuit of the G1-checkpoint can be disrupted by a variety of mechanisms during carcinogenesis. p53 is probably the most well-known and studied tumour suppressor and inactivating mutations are reported in $20-30 \%$ of breast cancers. Overexpression of the cell cycle promoting proteins cyclin D1 and cyclin E has been reported in 40-50\% respective $20-30 \%$ of invasive breast cancers [37]. The $M y c$ gene has been reported amplified and overexpressed in 15$25 \%$ of breast tumours and is associated with a poor prognosis [37].

\section{Metabolic reprogramming}

To enable the high proliferation and growth, cancer cells require an increased amount of energy. The metabolic switch of cancer cells from normal glycolysis followed by oxidative phosphorylation, to an aerobic glycolysis, was proposed by Otto Warburg already in the 1930s [38, 39]. The Warburg effect includes an increased glucose uptake by upregulation of GLUT1 receptors, to compensate for the least efficient way of producing ATP. The increased lactate production may in some tumours be utilised by a special tumour cell population adapted to usage of lactate as an energy source $[20,21]$. Recent findings suggest upregulation of common cancer pathways, including PI3K/AKT/mTOR to play a key role in the metabolic switch $[40,41]$. The AMPK/mTOR axis constitutes a central hub for the regulation of cellular growth and proliferation in response to nutrient, energy and oxygen availability, as well as growth factors including IGFs and insulin. In addition to an increased energy consumption, cancer cells also require cellular building blocks including nucleotides, fatty acids and proteins, which among others may be acquired through mTOR upregulation of the translational machinery [40]. 


\section{Apoptosis resistance}

The apoptotic programme is suggested to be present in latent form in all cell types, where it can be triggered by a variety of signals, including DNA damage, withdrawal of growth factors, viral infections or p53 expression [1]. The ability to avoid apoptosis is shared by most if not all tumour cells. Normally, cell surface receptors and intracellular sensors, monitoring the environment, are responsible for triggering the apoptotic machinery [42]. The extrinsic pathway regulates apoptosis positively or negatively through extracellular signals. Mainly, the TNF family members TNF $\alpha$ and FAS mediate death signals via TNF-R1 and the FAS receptor respectively. This can be balanced with antiapoptotic signals via growth factor signalling. The intrinsic programme may be induced in response to e.g. DNA damage, where the proapoptotic Bcl-2 members Bak, Bid, Bim, Bad and Bax (the last mentioned upregulated by p53) stimulate mitochondria to release cytochrome $\mathrm{C}$ (Figure 5). Cytochrome $\mathrm{C}$ in turn activates Apafl which initiates an effector caspase cascade finally leading to apoptosis. On the other hand, the antiapoptotic proteins Bcl-2, Bcl-XL and Bcl-W inhibit Cytochrome $\mathrm{C}$ release in a counteracting mechanism $[20,21]$.

In tumour cells, the most commonly used strategy for evading apoptosis, is probably through loss of p53 function by mutations, as mentioned in a previous section. Overstimulation of the PI3K/AKT pathway, which transmits antiapoptotic signals, is another causing factor. The apoptosis suppressor $B C L 2$ is a well-known oncogene, which has attracted much attention in the context of breast cancer since its regulation seems to be hormone dependent. Expression of the protein in human breast tissue has been shown to vary dramatically throughout the menstrual cycle, and the levels are increased in ER positive tumours [1]. In addition, ER-signalling in breast tumours was recently shown to mediate antiapoptotic responses by suppressing the expression of p53 targeted genes [43].

\section{Limitless replication}

Studies based on cell culturing have revealed that mammalian cells carry an intrinsic program that restricts the number of cell divisions, independently of the growth signalling pathways mentioned above [44]. The limiting factors are the telomeres; several thousands of short tandem repeats situated at the ends of all chromosomes. During each cell cycle, 50-100 bp of telomeric DNA are lost from each chromosome. When telomeres shorten to a critical length, normal cells in culture enter a nongrowing state called senescence. Disruption of the $\mathrm{pRb}$ signalling described above can circumvent this process and instead force the cells into a state of massive cell death and karyotypic disarray referred to as crisis. A few cells reach a state of immortalisation, the ability to multiply without limit. It is suggested that most cancer cells are immortalised, and achieve this state by maintaining the telomeres. Upregulated expression of telomerase, an enzyme capable of replicating telomeres and adding nucleotides to chromosome ends, is the most well-known strategy. In a few cases, a mechanism called alternate lengthening of telomeres (ALT), which is based on intrachromosomal changes, has been shown to serve the same purpose $[20,21]$.

The role of telomeres and telomerase in tumourigenesis may be considered as a double-edged sword, since telomere shortening, foremost as a result of normal ageing, is a key driver of genomic instability in combination with dysfunctional $\mathrm{p} 53$, promoting development of epithelial cancers [45]. 


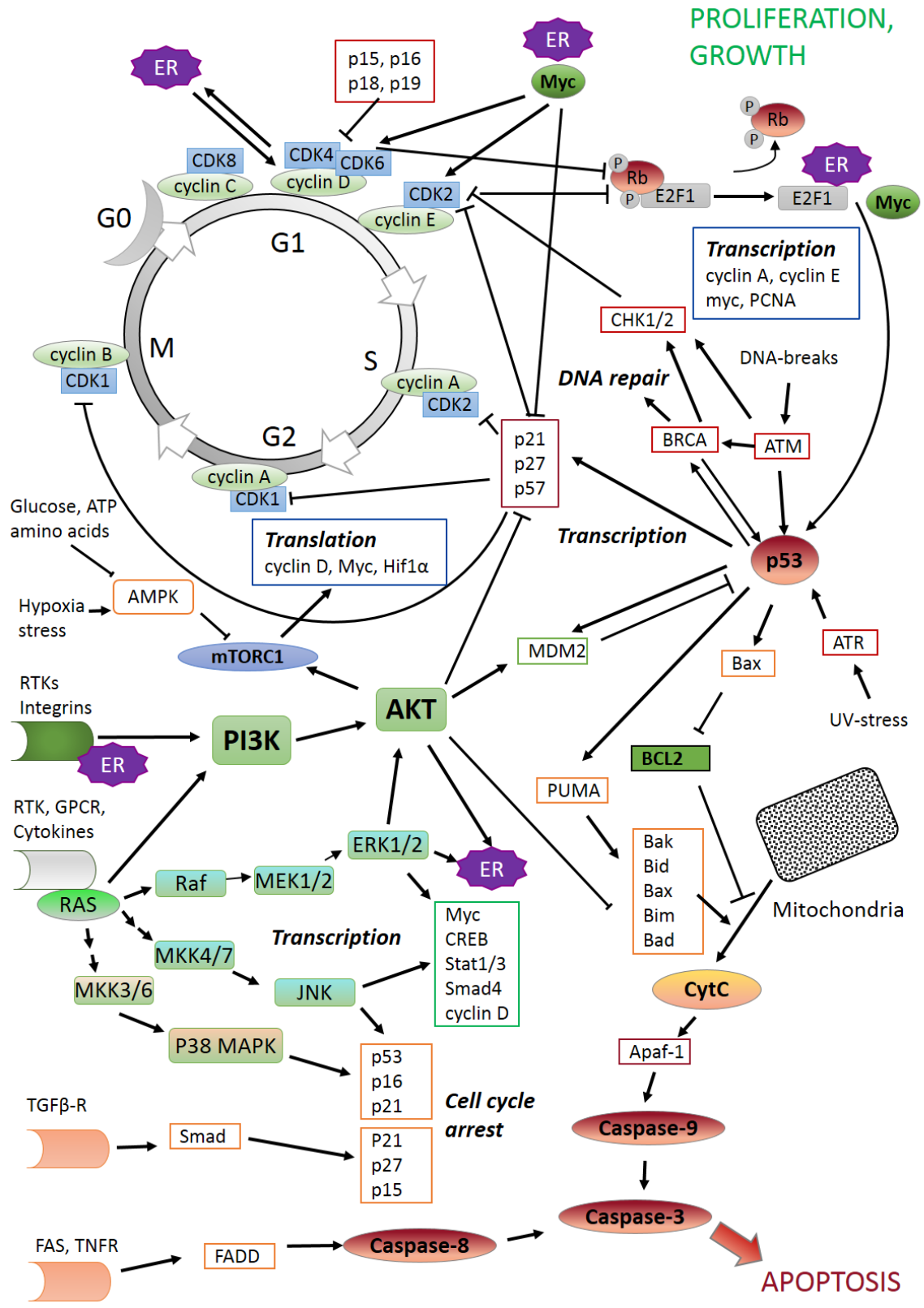

Figure 5 Overview of the main intracellular signalling circuits regulating growth, proliferation and apoptosis in breast cancer cells. 


\section{Role of the tumour microenvironment}

It is now accepted that tumours not solely constitute malignant cells, and may rather be considered complex organs consisting of several types of interplaying cells [20,21]. In addition to transformed epithelial cells, these are mainly endothelial cells in vasculature and lymphatic vessels, as well as vasculature supporting pericytes, cancer-associated fibroblasts (CAFs), and various types of immune cells. Inflammatory cells may secret proinflammatory cytokines and chemokines e.g. IL-1, IL-6 and CXCL12 involved in the angiogenetic switch. These can also stimulate recruitment of bone marrowderived stem cells, in turn enhancing proliferation of cancer stem cells. In breast cancer, the extracellular TGF $\beta$ is one of the most studied mediators of stromal-epithelial interactions. The cytokine is implicated to have a dual role, where epithelial TGF $\beta$, as previously mentioned, is considered a tumour suppressor due to its role in positively regulating apoptosis. However, stromal derived TGF $\beta$, possibly regulated by oestrogen, has been shown to promote EMT and thereby metastasis in later stage cancers [46, 47]. Increased local synthesis of oestrogen in breast tissue, mainly in stromal cells and adipocytes, is also assumed to stimulate malignant growth, among others through cross-talk to cytokine and chemokine signalling (Figure 6) [48, 49]. Thus, the complex crosstalk between different cells present in the developing normal gland also contributes in the tumourigenesis.

\section{Evading immune destruction}

The role for the immune system in cancer prevention is largely unknown, however it is suggested that the majority of arising tumour cells are immunologically detected and destroyed before further development [21]. In line with this, immunosuppressive treatment during organ transplantation increases the risk of certain cancer types e.g. skin and lung cancer. One newly suggested hallmark of cancer is the ability to avoid immune destruction. In breast cancer, mechanisms include downregulation of the surface markers HLA-1 and MICB (Figure 6), upregulation of antiapoptotic factors, production of proteinase inhibitors, or active induce of apoptosis in immune cells [50]. As described above, emerging evidence also show that the immune system may have a role not only in preventing, also in promoting cancer, through the secretion of proinflammatory cytokines and chemokines.

\section{Angiogenesis}

An essential factor for maintaining cell function and survival is the vasculature and its ability to supply the cells with oxygen and nutrients. Angiogenesis, the growth of blood vessels, is carefully and accurately regulated by several mechanisms. The angiogenetic switch is promoted by upregulation of VEGF, FGF and their respective receptors (Figure 6), as well as downregulation of the endogenous inhibitor thrombospondin-1. Intracellularly, increased Ras expression and loss of p53 function has been shown to be two of several underlying mechanisms. The bioavailability of angiogenesis activators and inhibitors can also be regulated by extracellular proteases e.g. by releasing FGF stored in the ECM [20]. In addition, a variety of stromal cells are involved in the angiogenesis [21]. 
Recently, it has been clear that the angiogenic switch occurs relatively early in the development of cancer and also in microscopic, premalignant tumours [21].

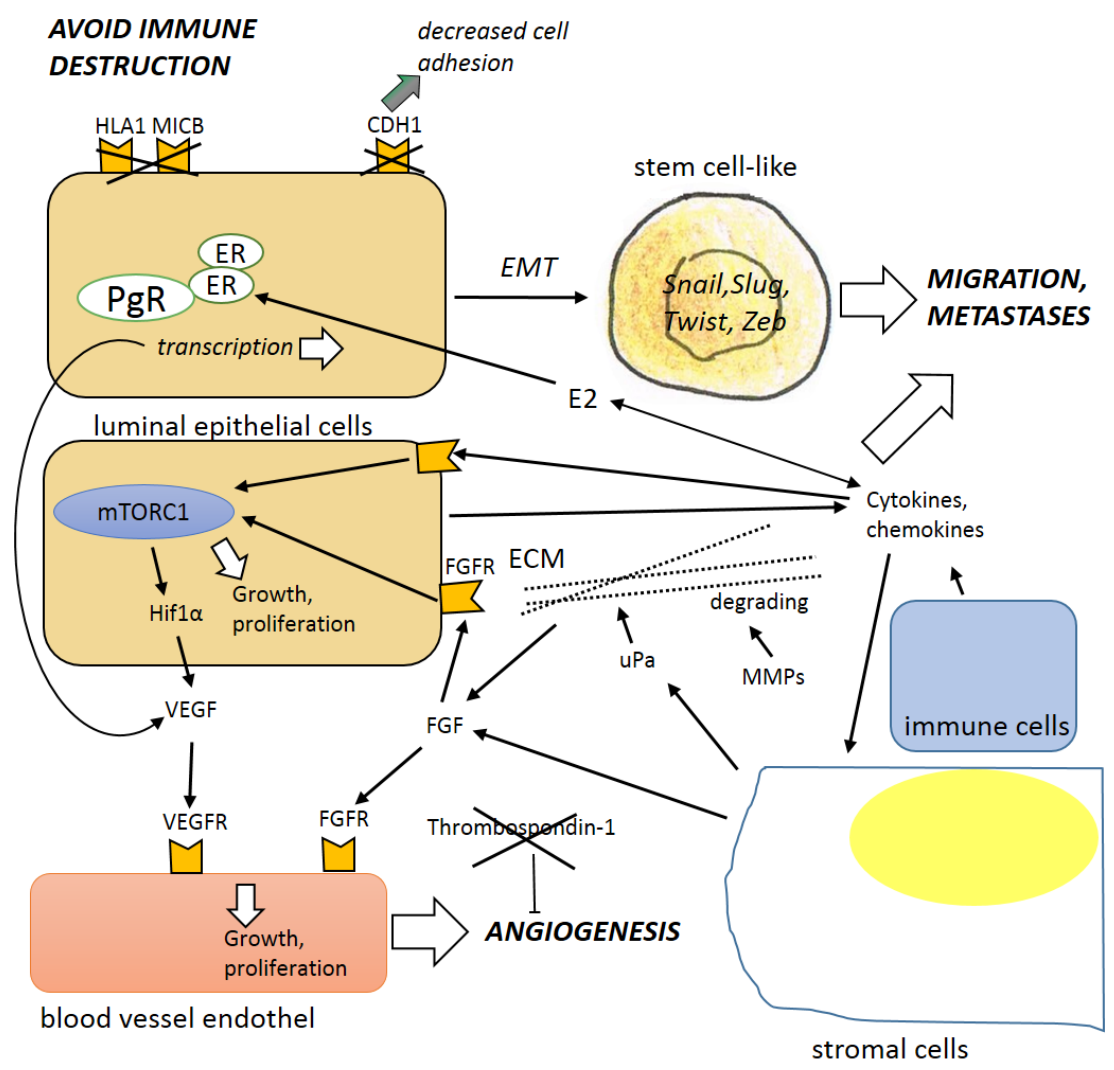

Figure 6 Overview of the main cell signalling circuits regulating angiogenesis, migration, metastasis and avoidance of immune destruction in cancer cells. 


\section{Metastasis}

It has been proposed that $90 \%$ of all cancer deaths are caused by metastases, rather than by the primary tumour [51]. By leaving the primary tumour, cells can form new colonies where space and nutrients are more accessible. The mechanisms behind metastasis are far from unravelled, however, it is suggested to involve changes in the adhesion of cells to their environments, as well as activation of ECM degrading proteases $[20,21]$ (Figure 6). E-cadherins are ubiquitously expressed on epithelial cells and mediate mechanical attachment between cells, as well as transmission of antigrowth signals, via $\beta$-catenin. The majority of cancers, including breast cancers have reduced or lost E-cadherin function by one of several mechanisms.

Matrix degrading proteases are believed to facilitate metastasis by clearing the way for cancer cells through the stroma, across blood vessel walls and epithelial cell layers [1]. Elevated expression of e.g. the UPA family of serine proteases is often detectable in tumour tissue and it is indicated that stromal and inflammatory cells are the main protease producers, yet the tumour cells probably induce this secretion $[20,21]$.

Emerging evidence suggests that the process by which epithelial cells can develop migratory properties may occur through EMT [20, 21]. During EMT, cells regress to a more undifferentiated state and acquire the migratory properties of mesenchymal cells, by upregulating expression of the embryonic transcription factors Snail, Slug, Twist and ZEB1/2, in turn down-regulating e.g. Ecadherin (Figure 6). The role of EMT for metastatic progression is still under debate [52]. However, increasing efforts are made in understanding the mechanisms behind the metastatic progress, which may be the most essential step to target in cancer progression.

\section{Genomic instability}

The tumour progression pathway can highly differ between cancers and the above mentioned capabilities can be developed at different times during the progression. However, it is clear that genomic changes play a major role for cells to develop malignancy. In normal cells, the advanced control-and repair systems are effective enough to make these multiple genomic changes unlikely to occur. It is therefore likely that in the early stage of cancer, the cells develop genomic instability, leading to increased mutability [20,21]. Genomic instability is suggested to result from primary mutations affecting the systems of replication and DNA repair. Defects in the DNA damage sensor p53 and its pathway, as mentioned above, is evident in most cancers. BRCA 1 and 2 are suggested to be involved in regulation of DNA transcription and repair (Figure 5), although the exact mechanisms are unclear. Genetic instability is in all probability one of the earliest hallmarks of cancer and may therefore be present even in tissue appearing to be histologically normal. However, whether inherited or sporadic, genomic instability leads to increased potential of developing further genetic changes, such as point mutations, chromosomal translocations, gene loss or amplifications [37]. 


\section{Gene amplification}

Genetic alterations are fundamental characteristics in the development of malignancy. Copy number aberrations of certain chromosomal regions are frequently found in solid tumours and are, with its subsequent changes in gene expression, expected to be essential for tumourigenesis. Gene amplification is a non-random event that refers to an increase in copy numbers of a restricted chromosomal region [53]. The amplicon is usually $0.5-10 \mathrm{Mb}$ in length and shall by definition not exceed $20 \mathrm{Mb}$ [54]. Amplification of larger chromosomal regions by aneuploidy or translocations, as well as a low copy number increase $(<4)$, is usually referred to as gain rather than amplification. Oncogenes can be amplified to a copy number up to 500 in tumour cells [55], yet it is well accepted that also deletions and low-level gains are of importance in cancer pathology [54]. Gene amplification often leads to enlarged quantity of the corresponding mRNA and protein $[56,57]$ and seems to occur non-randomly, suggesting that amplicons harbour genes essential for tumour progression. Identifying patterns of gene amplification in tumours therefore has prognostic as well as diagnostic usefulness and could be utilised as a guide for treatment design [53]. Gene amplifications usually appear in advanced cancers, probably as a result of genomic instability, and could therefore be indicators of high grade and poor prognosis [54]. Increasing numbers of amplifications in a tumour have been associated with worse prognosis $[58,59]$. In addition, certain patterns of gene amplification seem to be cancer type specific [54], and might therefore be valuable as independent classification parameters.

The value of gene amplification analysis in clinical practice is becoming more and more evident. Numerous specific amplicons, exemplified by these harbouring the ERBB2/HER2 (17q12), MYC (8q24), CCND1 (11q13), AIB1 (20q12), FGFR1 (8p12), EGFR (7p12) and MDM2 (12q14) genes in breast cancer, have been shown prognostically significant [58]. Genes in the centre of amplicons are candidate drivers of tumourigenesis, and since the tumour therefore could be addicted to their enhanced expression, they are interesting therapeutic targets [53]. So far, there are few available drugs directed towards amplification-activated oncogenes, nonetheless, many possible targets are under investigation.

Of importance for the present project, the genes encoding the mTOR targets S6K1, S6K2 and 4EBP1 are localised in amplification hotspots at $17 \mathrm{q} 23,11 \mathrm{q} 13$ and 8p12, respectively. The RPS6KB1 gene has earlier been shown amplified in breast cancer, associated with a poor prognosis [60-63]. In this project, we also report clinical significance of amplification of the RPS6KB2 gene at 11q13, as well as EIF4EBP1 at 8p12. The following section will describe discovery and clinical relevance of the 11q13 amplicon in breast cancer, concluding with its relations to $8 \mathrm{p} 12$ amplification. 


\section{Mechanisms of gene amplification}

Amplicons can appear intrachromosomally at the site of origin, or as extrachromosomal DNAfragments that lack a centromere and replicate independent of the chromosomes. Intrachromosomal amplicons were first observed cytogenetically by FISH and are therefore referred to as homogeneously staining regions (HSR). When sufficiently large, extrachromosomal amplicons can be visualised by FISH as double minutes (DMs), whereas molecular biology tools are needed to recognise the submicroscopic DNA fragments designed episomes [54]. Amplicons might contain mixtures of DNA fragments from different chromosomal locations and can be distributed in various forms throughout the genome [53].

The mechanisms behind amplicon formation are not fully established, however, several models explaining the amplification process are suggested [64]. The breakage-fusion-bridge (BFB) model proposed by McClintock is the main explanation for HSR formation [65]. The process is initiated by telomere loss or other double-strand breakages (Figure 7), which promotes fusion of the sister chromatids after the following DNA-replication. The result is a dicentric chromosome that forms a bridge during anaphase and is prone to break again, thereby initiating a new BFB cycle. The process can repeat in several subsequent divisions, until the telomeres are stabled by e.g. translocation to another chromosome, resulting in an amplicon with amplification units arranged as inverted repeats. At the same time, the DNA sequences distal to the amplicons are commonly deleted or translocated [53].
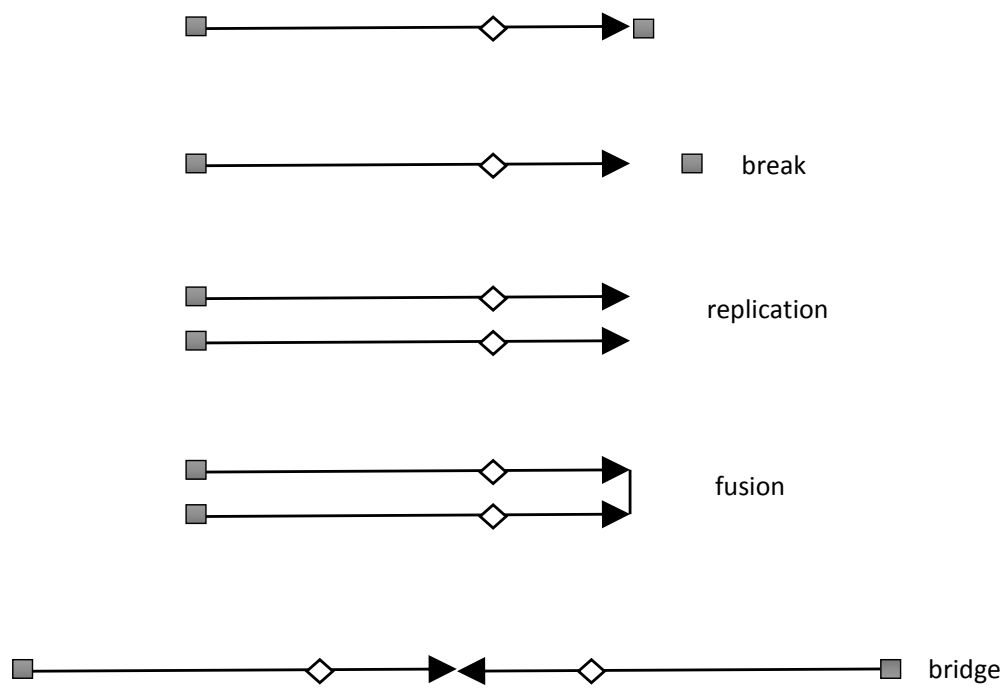

Figure 7 The breakage-fusion-bridge (BFB) model explaining the mechanism of gene amplification. Following the loss of a telomere, sister chromatids fuse. During anaphase, the resulting dicentric chromosome forms a bridge, which might break again, initiating a new BFB cycle. 
According to the excision-segregation model, extrachromosomal fragments are formed by looping out from the chromosomes. This happens through breakage of replication bubbles. The fragments can also be formed secondary, during breakdown of HRSs whereas HRSs in some cases can be composed of DMs inserted into a regular chromosome [64].

The genomic site-specificity of structural chromosomal alterations has also been suggested to be influenced by fragile sites; chromosomal regions that are damage sensitive to chemicals that affect replication [54]. Statistically, it has been shown that fragile sites often appear at the ends of amplicons, however, at the same time these sites seem to be distributed equally on amplified and nonamplified regions [66]. Fragile sites in the proximity of the gene under selection may be necessary, nevertheless the genomic localisations of amplicons are likely also determined by the sites of oncogenes, as described in the next section.

\section{Amplicons as indicators of tumour driving oncogenes}

It is increasingly evident that specific amplicon formation is a result of selective pressure during tumour development. This was first discovered by in vitro studies, where cell lines were shown to develop cytotoxic resistance by upregulating drug target genes through amplification [67]. Another strong argument is the observation that the locations of gene amplifications are not randomly distributed across the genome, and that known oncogenes often colocalise with amplification hotspots [54]. Defining and mapping amplicons are therefore of great interest in the search for new oncogenes promoting growth advantage and other tumour characteristics. Recently developed array based techniques are now available for whole-genome analysis of gene amplification patterns, allowing a comprehensive view of amplicon boundaries as well as identification of recurrent regions of amplifications. Amplicons usually harbour several genes, and thus pinpointing a specific driver gene is a big challenge. It is assumed that under selective pressure, the amplicon can be narrower with focus on the driver gene, why it is suggested that the genes of interest should be situated in the region of maximal amplitude. However, subsequent investigations on whether candidate oncogenes actually are expressed in the tumour, if amplification is coherent with overexpression and in the long run, if its amplification status has prognostic and predictive value for clinical outcome, are also necessary [53]. 


\section{The 11q13 amplicon in breast cancer}

Amplification of the 11q13 chromosomal region was discovered and connected to breast cancer for almost two decades ago, and has thereafter been extensively studied. A heterogeneous amplification pattern, several amplification cores and an extremely gene dense region make the search for candidate oncogenes a complicated process. The following section will describe the discovery of the 11q13 amplicon in breast cancer, comparisons with its amplification patterns in other cancers, associations with breast cancer subtypes, candidate oncogenes in the region and its coamplifications with $8 \mathrm{p} 12$.

\section{The discovery of 11q13 amplification}

In the late 1980s, studies on mice indicated that a virus, now referred to as mouse mammary tumour virus (MMTV), could give rise to mouse mammary tumours by activating the fibroblast growth factors fgf3 and fgf4 (reviewed in [68]). Further studies confirmed a role of the genes in mouse mammary tumourigenesis and this prompted interest in linking these findings to human breast cancer. The human $F G F 3$ was subsequently mapped to $11 \mathrm{q} 13$ and in connection to this, it was revealed that the gene is amplified in a significant proportion of breast carcinomas. Shortly afterwards, the $F G F 4$ gene was shown to be situated at the same chromosomal region and coamplified with $F G F 3$ in breast and oesophageal tumours. However, when corresponding mRNA levels were measured, it turned out that the genes were more or less transcriptionally inactive in breast tissue and an increase in DNA copy number did not change this fact. Thus, FGF3 and FGF4 seemed to be passenger genes, and the search for a new driver oncogene in the $11 \mathrm{q} 13$ region was initiated. The $11 \mathrm{q} 13$ region had also in other contexts been implicated in tumourigenesis. In B-cell lymphomas, this region was shown involved in translocation with chromosome $14, \mathrm{t}(11 ; 14)$ and a hypothetical oncogene at 11q13 was referred to as B-cell leukaemia 1 (BCL1) [69]. In rare cases of parathyroid adenoma, a chromosomal inversion places 11q13 adjacent to 11p15, leading to increased expression of an 11q13 located gene referred to as Parathyroid Adenomatosis 1 (PRAD1) [70]. In the early 1990s, several groups studying cell cycle regulation simultaneously identified and isolated a gene whose corresponding protein was shown to promote progression through the G1 phase of the cell cycle. The gene, mapped to 11q13, was referred to as $C C N D 1$ and its corresponding protein; cyclin D1 ([68] and references therein). It soon became clear that BCL1, PRAD1 and CCND1 were the same gene, and a new candidate oncogene in the $11 \mathrm{q} 13$ region was introduced. Hitherto, all markers used for studies of 11q13 amplification had been placed in the proximity to the CCND1 gene. However, when the region of study was extended, it turned out that the amplicon actually spanned a much larger area. Subsequently, Shuuring et al. investigated the expression of genes within the area and clarified the CCND1 gene and also the EMS1 gene as being overexpressed as a consequence of amplification [71]. To date, the CCND1 gene is still the far most extensively studied candidate oncogene in the 11q13 region in breast cancer, though studies have revealed several additional amplicons, suggesting further driver oncogenes in the area [72]. 


\section{1q13 amplification in different cancer types}

It is now well established that the chromosome band 11q13 is amplified in a significant proportion of primary breast tumours. Observed frequencies range from $10 \%$ up to $25 \%$, depending on selection of patient cohorts, applied methodology and choice of markers [58, 73-78]. Amplification of this region has been reported to the same extent in DCIS and benign breast tumours [79-81], suggesting that this is an early event, connected to initiation of carcinogenesis, rather than a secondary effect of genomic instability. This event is not restricted to breast cancer and has also been found in other cancers, mainly ovarian cancer, oral- (OSCC) and head-and-neck squamous cell carcinoma (HNSCC), where up to $36 \%$ has been reported [82]. The patterns of amplification seem to vary between different cancers. Whereas CCND1 and the area in its proximity constitutes the main amplicon in this region in breast cancer, this gene is rarely amplified in ovarian cancer, where amplicons usually arise more distally [83].

\section{Amplification patterns and candidate oncogenes at 11q13}

Originally, it was thought that one single amplicon, spanning numerous megabases $(\mathrm{Mb})$, represented all 11q13 amplifications. However, it is now evident that the region harbours several amplicon cores that can be amplified independently or together in different combinations. Ormandy and colleagues describe four core regions [84], whereas Albertson suggests up to eight independent amplicons [53]. Consequently, this implies that the region contains several genes with the potential to drive tumourigenesis. The nature of the amplicons vary a lot from case to case and may consist of one or several specific amplicons, or a large amplicon spanning the whole region. In addition, many 11q13 amplifications are accompanied by a deletion of the distal part of the q-arm, which is interesting in several aspects. This area of $11 \mathrm{q}$ harbours several potential tumour suppressor genes, whose deletion could facilitate cancer progression. Furthermore, the occurrence of telomere loss suggests that BFB cycles could underlie the formation of these amplicons, and the amplicons usually occurs as inverted duplicated segments [85]. 11q13 is an extremely gene dense region and up to date approximately 500 genes are known to be situated in the area. Therefore, distinguishing potential amplicon drivers remains a challenge.

\section{Implicated associations with subtypes and amplification patterns}

It is now more or less stated that 11q13 amplification is connected to positive ER status. A strong correlation between 11q13 amplification and Luminal subtype, as well as an inverse correlation to basal-like expression type have also been shown [77, 86]. Although cyclin D1 may be the prime candidate for this connection, the same tendency is seen for several independent amplification cores in this region, making the mechanisms behind this connection yet unclear. Due to the availability of antioestrogen therapies, ER positivity is at present considered as a marker of good prognosis. However, among patients with ER positive tumours, these also harbouring 11q13 amplification seem to constitute a subgroup with higher grade and a relatively poor prognosis [58, 74]. When considering histological subtypes, 11q13 amplifications have been shown most prevalent in lobular breast carcinomas $[87,88]$. 
The presence of one amplicon increases the likelihood of developing additional amplifications, probably partly as a result of genomic instability [58]. These multiple amplicons can be associated with each other, both physically and statistically. Several studies have demonstrated that the 11q13 region often is coamplified with regions on 8p11-12 [59, 77]. The presence of both of these amplifications seems to predict a worse patient outcome than either amplicon alone, suggesting a synergy between them [59]. 8p12 is frequently amplified in breast cancers and a main candidate driver oncogene for this region has been FGFR1 [89]. However, as in the 11q13 case, 8p12 harbour several amplicons that can be amplified independent or together [90], indicating more than one driving oncogene. Paterson et al. investigated the physical structure of the 11q13-8p12 coamplifications in some breast tumours and breast cancer cell lines and demonstrated that a variety of arrangements can occur. This includes amplifications of a translocation junction, separate amplifications of the regions on different chromosomes and also in a few cases on the same chromosome, further strengthening the probability of multiple independent targets on the two regions [91].

In the present project, results suggest the mTOR targets S6K2 and 4EBP1 as candidate oncogenes for the 8 p12/11q13 synergy. The following sections will describe the implicated roles of the $\mathrm{mTOR} / \mathrm{S} 6 \mathrm{~K} / 4 \mathrm{EBP} 1$ pathway in breast cancer. 


\section{The $m T O R / S 6 K / 4 E B P 1$ pathway in breast cancer}

The mTOR is assumed to be a critical effector in several cellular functions commonly deregulated in cancer [92]. The PI3K/AKT signalling pathway, a major regulator of mTOR function, is the far most altered pathway in breast cancer and is estimated to be constitutively activated in $>70 \%$ of cases [93]. In response to growth factors, hormones, nutrients, hypoxia and energy (ATP) the $\mathrm{PI} 3 \mathrm{~K} / \mathrm{AKT} / \mathrm{mTOR}$ pathway regulates cell growth, proliferation and metabolism through translational control of essential proteins. Recently, it was shown that humans carrying mutations in the growth hormone receptor leading to reduced IGF/mTOR signalling are resistant to development of malignant tumours, suggesting this pathway as one major factor behind the origin of cancer [94]. The following section describes the molecular basis of RTK/PI3K/AKT/mTOR signalling, what is known about its role in breast cancer, and its implicated role in endocrine resistance.

\section{Receptor tyrosine kinases as main regulators of $\mathrm{PI} 3 \mathrm{~K} / \mathrm{AKT} / \mathrm{mTOR}$ signalling}

The human kinome encompasses 518 known protein kinases, divided into ten families, with a wellconserved kinase domain structure $[95,96]$ (Table 2, Figure 8). Kinases are estimated to regulate the function of $>50 \%$ of all cellular proteins. The first crystal structure of a kinase domain was represented by PKA in 1991. The RTKs are highly conserved from C. elegans to human and have been shown as key regulators of many cellular functions [97]. To date, 58 human RTKs have been described, and divided into 20 subfamilies (Figure 9). This section will shortly describe the known mechanisms of RTK signalling, with focus on the EGFR, FGFR, IGFR and IR families, which are the major RTKs implicated in breast cancer and regulators of mTOR signalling.

Table 2 Overview of the human kinome.

\section{Protein kinase families}

-AGC: cAMP, cGMP-dependent and protein kinase $\mathrm{C}$

e.g. AKT, PDK, S6K

-CAMK: calcium/calmodulin dependent

-CK1: casein kinases

-CMGC: CDK, MAPK, GSK3, CLK

-PKL: protein kinase like

PIK: PI-3 kinases

PIKK: PI-3 kinase like, e.g. mTOR
-RGC: receptor guanylate cyclases

-STE: sterile 7, 11 and 20 kinases

-TK: tyrosine kinase

e.g. RTKs

-TKL: tyrosine kinase like

-Atypical/other 
$\mathbf{N}$-terminal lobe, mainly antiparallel $\beta$-sheets

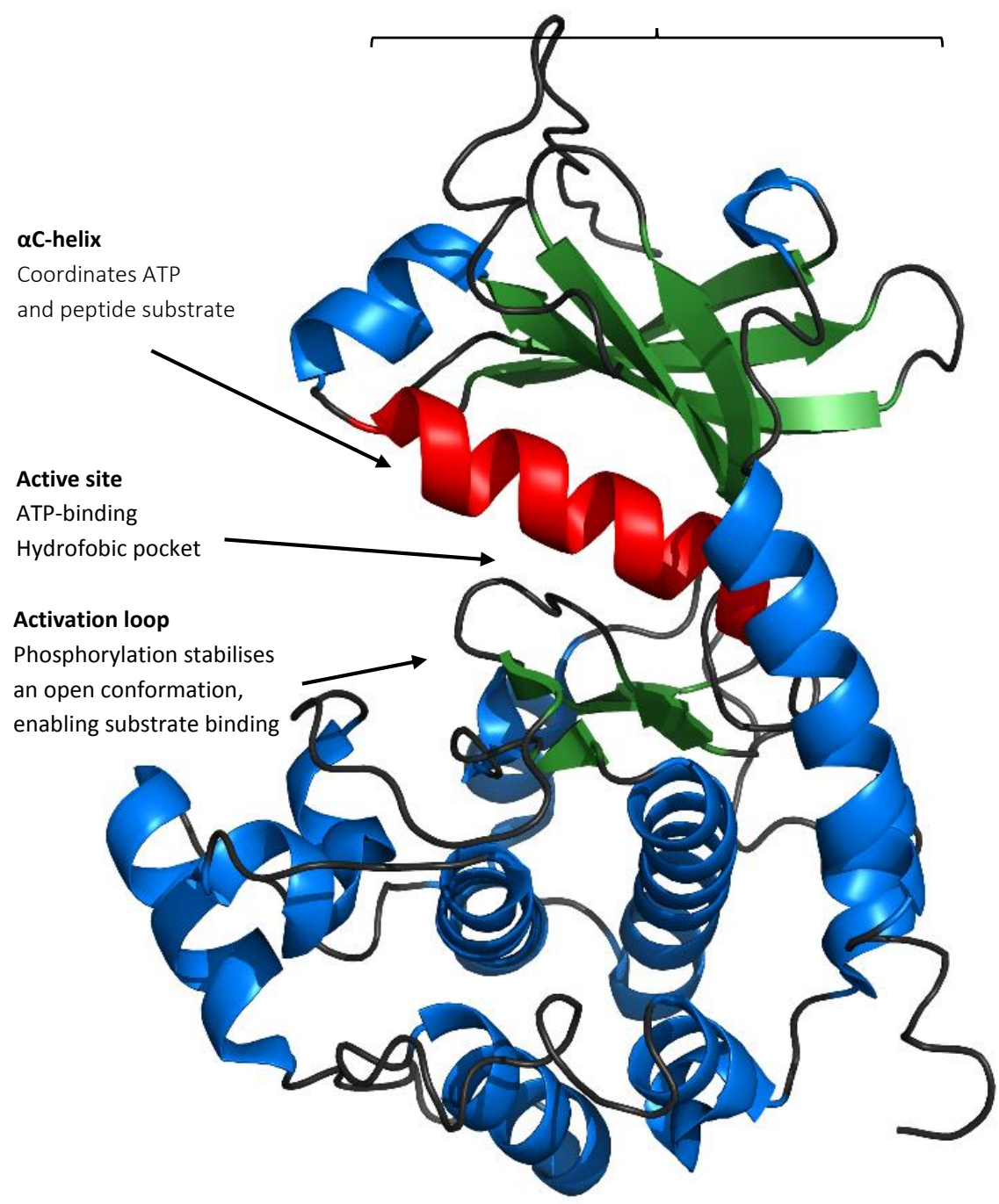

C-terminal lobe: $\alpha$-helices

Figure 8 Overview of the conserved kinase domain structure exemplified by protein kinase A (PKA). 


\section{Receptor tyrosine kinase structure and regulation}

All RTKs possess a common structure with an extracellular ligand binding domain, a single transmembrane helix and a cytoplasmic tyrosine kinase domain including additional C-terminal and juxtamembrane regulatory regions (Figure 9). Several different mechanisms of ligand binding and activation have been evident [98]. In general, ligand binding to the extracellular domain of the receptor facilitates dimerisation and subsequent transphosphorylation at intracellular tyrosine residues, in turn allowing recruitment and activation of downstream intracellular signalling pathways.

After activation, a second phase of transphosphorylation occurs, forming several phosphotyrosines as docking sites for downstream molecules. For IGFR, IRSs are the sites for the second phase phosphorylation. In turn, phosphorylation of IRS can be regulated through several positive and negative feedback mechanisms. Primarily, docking of Src homology 2 (SH2) and phosphotyrosine binding proteins occurs, including IRS (IGFR and IR), FRS2 (FGFR and NGRs) or Gab1 (several RTKs). These may in turn be phosphorylated by the tyrosine kinase, facilitating recruitment and activation of additional proteins, several containing SH3, WW, PDZ, PH, PV, C1, C2 or FYVE domains.

RTK signalling constitutes extremely complex networks, with numerous feedback mechanisms, where multiple factors and conditions determine the final outcome, including the amount of different ligands and receptors and downstream factors. This is also balanced with negatively regulating mechanisms, mainly RTK dephosphorylation by protein tyrosine phosphatases (PTPs), receptor internalisation through clathrin-mediated endocytosis, downregulation by ubiquitination as well as downstream cellular feedback mechanisms. 


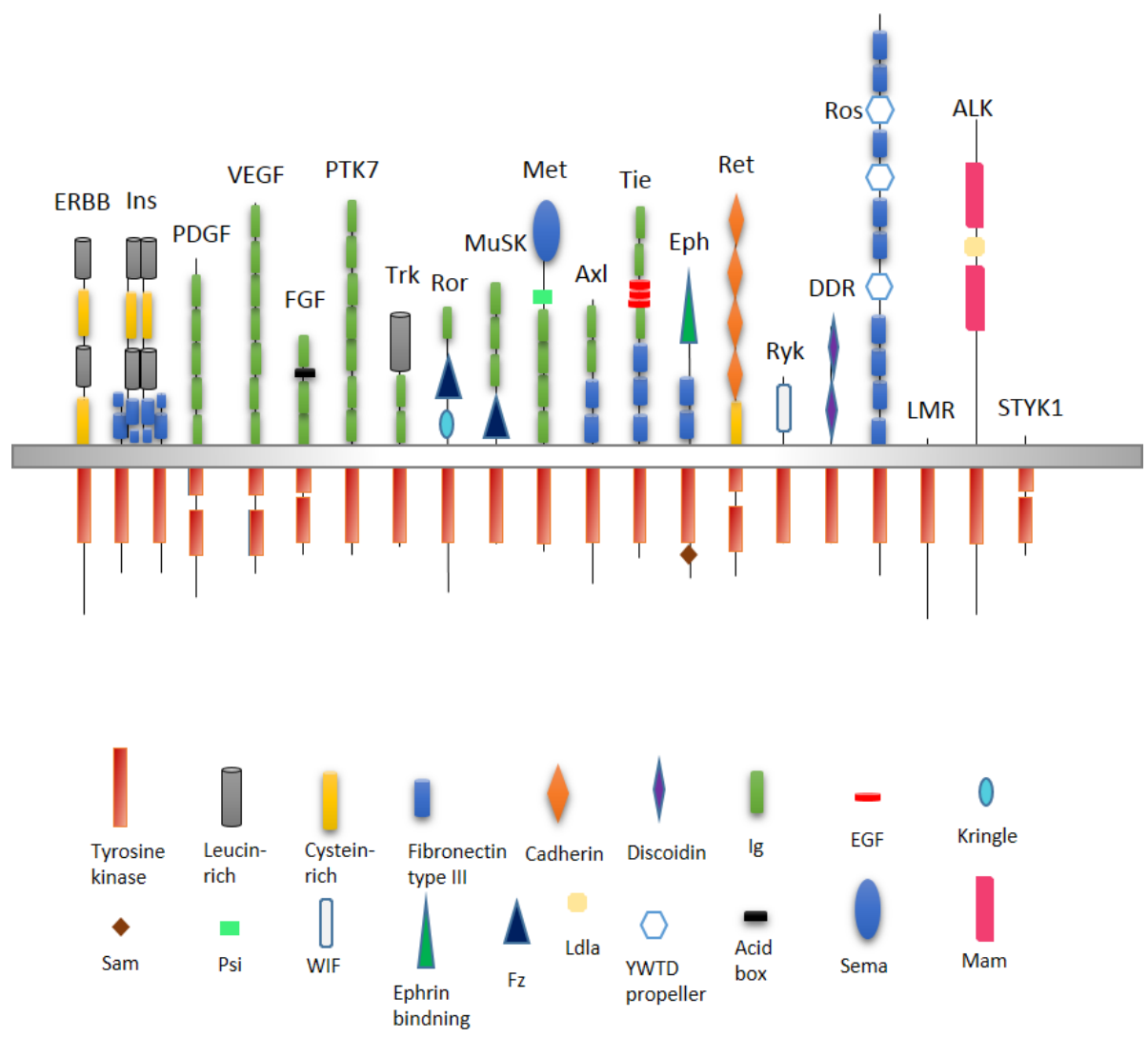

Figure 9 Domain structures of the 20 known receptor tyrosine kinase families [98]. (For further explanations see list of abbreviations). 


\section{$\mathrm{PI} 3 \mathrm{~K} / \mathrm{AKT}$ signalling is the most overstimulated pathway in breast cancer}

The PI3K was first discovered in the 1980s by Lewis Cantley and colleagues, searching for kinases with activity towards phosphoinositoles, in mouse $3 \mathrm{~F} 3$ cells $[99,100]$. In humans, Volerina et al. cloned and mapped the PIK3CA gene to 3q26 in 1994 [101]. To date, three classes of PI3Ks are known, where class I is the most well-studied and foremost implicated in oncogenesis [102], and will therefore primarily be considered in the context of this project. The class I PI3Ks constitute a heterodimer with one catalytic subunit, p110, encoded by the PIK3CA, PIK3CB, PIK3CG or PIK3CD genes and one regulatory subunit, p85, encoded by PIK3R1, PIK3R2, or PIK3R3.

The p85 regulatory subunit contains $\mathrm{SH} 2$ and $\mathrm{SH} 3$ domains, facilitating interactions with phosphotyrosines at activated RTKs. Subsequently, PI3K reaches close proximity with the plasma membrane, and after activation by conformational changes, PI3K phosphorylates the inositol ring 3'$\mathrm{OH}$ group of inositol phospholipids in the plasma membrane, generating the turnover of phosphatidylinositol-4,5-bisphosphate $\left(\mathrm{PIP}_{2}\right)$ to phosphatidylinositol-3,4,5-trisphosphate $\left(\mathrm{PIP}_{3}\right)$. The reverse reaction is generated by $\mathrm{PTEN}$. $\mathrm{PIP}_{3}$ becomes docking site for other signalling molecules, including the serine/threonine kinases AKT and PDK1 (Figure 10).

Three members of the PKB/AKT family, with $80 \%$ sequence homology have been characterised to date, named PKB $\alpha$ (AKT1, 14q32), PKB $\beta$ (AKT2, 19q13) and PKB $\gamma$ (AKT3, 1q44). In most tissues, AKT1 is the predominant isoform, whereas AKT2 mainly is located in insulin responsive tissues and AKT3 is foremost expressed in neuronal tissue (reviewed in [103]).

$\mathrm{AKT}$ and $\mathrm{PDK} 1$ bind $\mathrm{PIP}_{3}$ via their pleckstrin homology $(\mathrm{PH})$ domains. Activation of $\mathrm{AKT}$ is then mediated by PDK1 phosphorylation of T308. A second phosphorylation on S473, mediated by mTORC2 is required for complete AKT activation. Activated AKT may be translocated to the nucleus, or remain in the cytoplasm for activation of numerous downstream actors, regulating several key cellular mechanisms. These include cell cycle progression, apoptosis, transcription and translation [104], the latter in which mTOR plays a key role (Figure 10). 


\section{mTOR signalling from the two complexes mTORC1 and mTORC2}

The serine/threonine kinase mTOR was initially identified in the early 1990s as the cellular target of the immunosuppressor and antitumour agent Rapamycin [105]. Ever since, multiple cellular functions of mTOR and its complex signalling circuit have been uncovered. The gene encoding mTOR is located at the chromosomal region $1 \mathrm{p} 36$. Recently, the crystal structure of the mTOR kinase domain was reported [106]. It is now known that mTOR exists in two cellular complexes, referred to as mTORC1 [107] and mTORC2 [108], both possessing essential cellular functions.

\section{The mTORC1 is a main integrator of tissue homeostasis}

mTORC1 is composed of mTOR, Raptor, mLST8, PRAS 40, and FKBP38 [109]. In response to growth factors, hormones, nutrients, hypoxia and energy (ATP) mTORC1 regulates cell growth, proliferation and metabolism through translational control of essential proteins (Figure 10). As mentioned previously, two well-known mitogenic stimulators of mTOR function are the PI3K/AKT and Ras/MAPK cell signalling pathways, which regulate mTORC1 via phosphorylation and inhibition of the TSC1-TSC2 complex. TSC1-TSC2 in turn inhibits the GTPase Rheb. Active GTP bound Rheb activates mTORC1 in a partly unknown mechanism, by binding FKBP38 and the mTOR kinase domain, inducing an active conformation. mTORC1 activation is also promoted by phosphorylations of the mTOR kinase domain at several sites. Efforts in characterisation of responsible kinases for mTOR regulation have suggested AKT, S6K as well as autophosphorylation by mTOR itself, however, information is scarce $([110,111]$ and references therein). The Wnt signalling pathway has also been implicated as an upstream regulator of mTOR function, possibly through inactivation of GSK-3 (Figure 10) [112]. During hypoxia, mTORC1 function is diminished by Redd1/Redd2, in turn upregulated by hypoxia inducible factor (HIF1 $\alpha$ ) [109]. The availability of energy and nutrients is also critical for mTORC1 function. A low ATP/AMP ratio results in activation of AMPK via LKB1 phosphorylation. AMPK in turn, phosphorylates TSC2 resulting in mTORC1 inactivation. The mechanisms behind nutrient stimulation of mTOR are far from unravelled, though it is suggested that a high level of branched amino acids, in particular Leucine, activates mTORC1 by upregulating among others the Rag GTPases. The most well-known substrates of mTORC1 are the p70 ribosomal S6 kinases $\mathrm{S} 6 \mathrm{~K} 1$ and $\mathrm{S} 6 \mathrm{~K} 2$, and 4EBP1, which are involved in regulation of the translational machinery [113].

\section{mTORC2 regulates cytoskeletal dynamics as well as AKT}

Whereas numerous upstream regulators of $\mathrm{mTORC} 1$ function have been identified, the mechanisms behind mTORC2 activation are less known. mTORC2 is shown phosphorylated and activated in response to growth factors, though the intracellular pathways are still unravelled [92]. In addition to mTOR, mTORC2 consists of mLST8, mSIN1, PROTOR and Rictor. mSIN1, in turn exists in three different isoforms, therefore three distinct $\mathrm{mTORC} 2 \mathrm{~s}$ can be defined, possibly with divergent properties. The complex has been implicated in regulation of cytoskeletal dynamics, through activation of Rho GTPases and PKC $\alpha$. mTORC2 has now been revealed as the earlier unknown kinase responsible for phosphorylating AKT at S473, thereby promoting its activation [114]. 


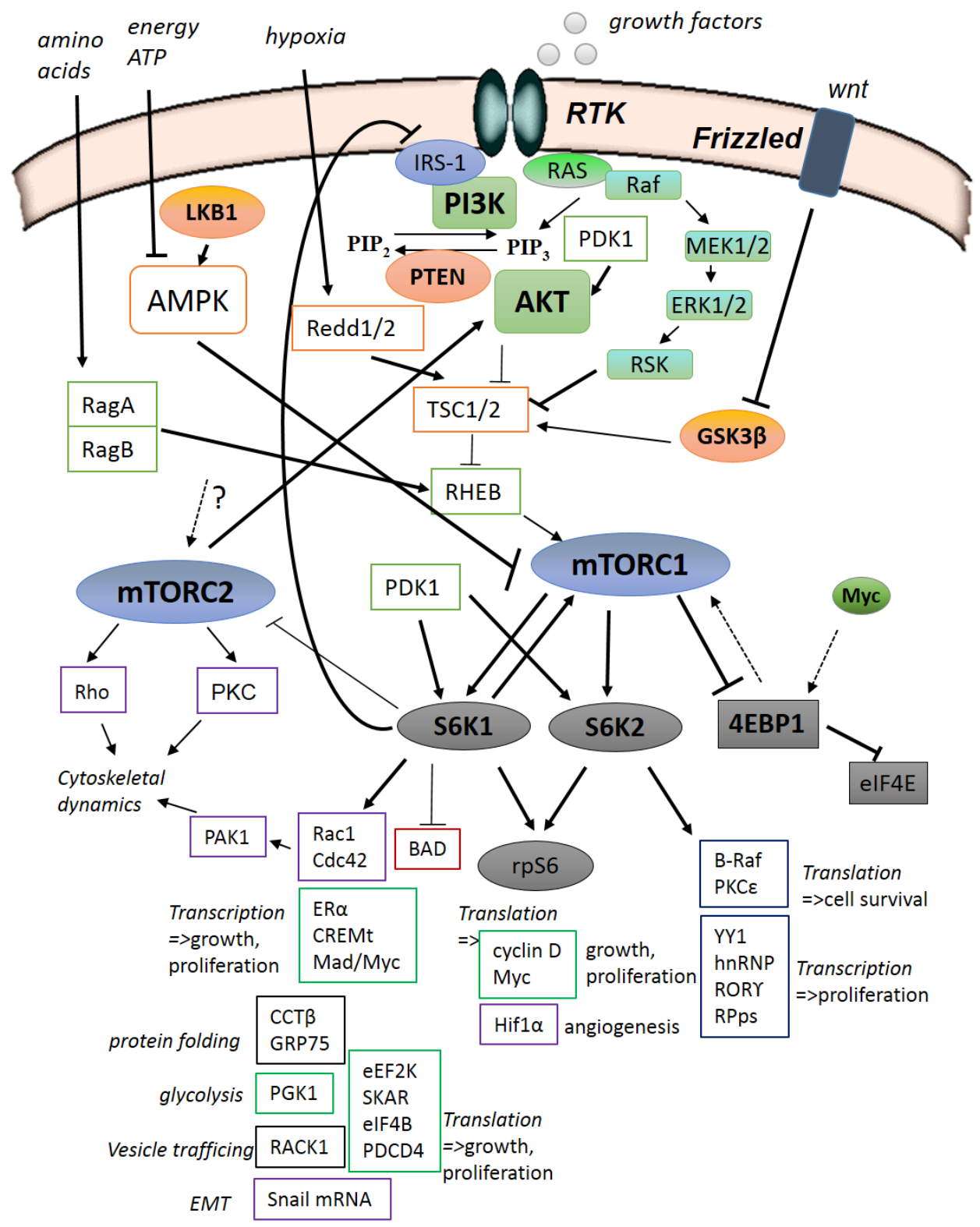

Figure 10 Overview of the RTK/PI3K/AKT/mTOR/S6K/4EBP1 signalling pathway. Suggested substrates and interaction partners of S6K1 and S6K2, respectively, are shown [115-121]. 


\section{The mTOR targets S6K1, S6K2 and 4EBP1}

Increased cellular proliferation is one of the hallmarks of cancer. To maintain a high proliferation, transformed cells commonly upregulate the translational machinery, resulting in an increased rate of protein synthesis, enabling the cells to double in size before division. As a result, histological examinations of highly transformed cells show enlarged nucleoli, where the ribosome biogenesis occurs [122]. The process of ribosome biogenesis is highly complex and energy consuming and it has been shown that in actively growing cells, ribosome biogenesis require $>70 \%$ of their total gene transcription. This involves coordinated regulation of the three RNA polymerases (I-III) for production and processing of four ribosomal RNAs, 80 ribosomal proteins as well as more than 200 additional proteins and non-coding RNAs involved in the process ([123] and references therein).

In normal cells, the mTORC1 pathway is a major regulator of protein synthesis by phosphorylating several factors in the translational initiation complex [124]. The next section describes the discovery of S6K1, S6K2 and 4EBP1, which are the main targets of mTORC1 phosphorylation, its cellular roles and implications in breast cancer.

\section{Discovery of S6K1 and S6K2, homologous AGC kinases}

Ribosomes, essential for the translational machinery, are in higher eukaryotes composed of the $60 \mathrm{~S}$ (large) and 40S (small) subunits. 60S consists of the three RNA molecules 5S, 5.8S and 28S together with approximately 45 proteins, whereas $40 \mathrm{~S}$ comprises the $18 \mathrm{~S}$ rRNA and 33 proteins. The ribosomal protein $\mathrm{S} 6$ (rpS6) in the $40 \mathrm{~S}$ subunit was the first discovered ribosomal protein to be regulated by phosphorylation [125]. The phosphorylation of rpS6 is now a well-established response to mitogens, and the mechanisms of phosphorylation have been studied thoroughly [115]. The first identified S6 enzymes were the p90 ribosomal S6 kinases (in mammals RSK1-4), responsible for S6 phosphorylation in Xenopus oocytes. However, subsequent studies led to the discovery of the p70 ribosomal S6 kinase, as the major S6 kinase in somatic cells [126, 127]. The RPS6KB1 gene, encoding S6K1, was first cloned in rat and rabbit [128-130] and shortly after the human orthologue located at the chromosomal region 17q23, was discovered and shown to encode two splicing variants, p70 and p85S6K1 [131]. At the end of the 1990s, a close paralogue of S6K1, termed S6K2, was cloned by several groups [132-135]. The RPS6KB2 gene, encoding S6K2 is located at the chromosomal region 11q13. To date, three main S6K1 isoforms and two S6K2 isoforms are known, and the proteins are together referred to as the p70 ribosomal S6 kinases [115], (Figure 11).

The serine/threonine kinases S6K1 and S6K2 belong to the cAMP-dependent, cGMP-dependent and protein kinase $C$ (AGC) family of kinases, also including among others PKA, AKT, PDK1, SGK and p90S6K. The AGC family members have a high homology in their catalytic domains and become active after phosphorylation by PDK1 at a Tyrosine residue in the activation loop (Figure 8) [115]. 


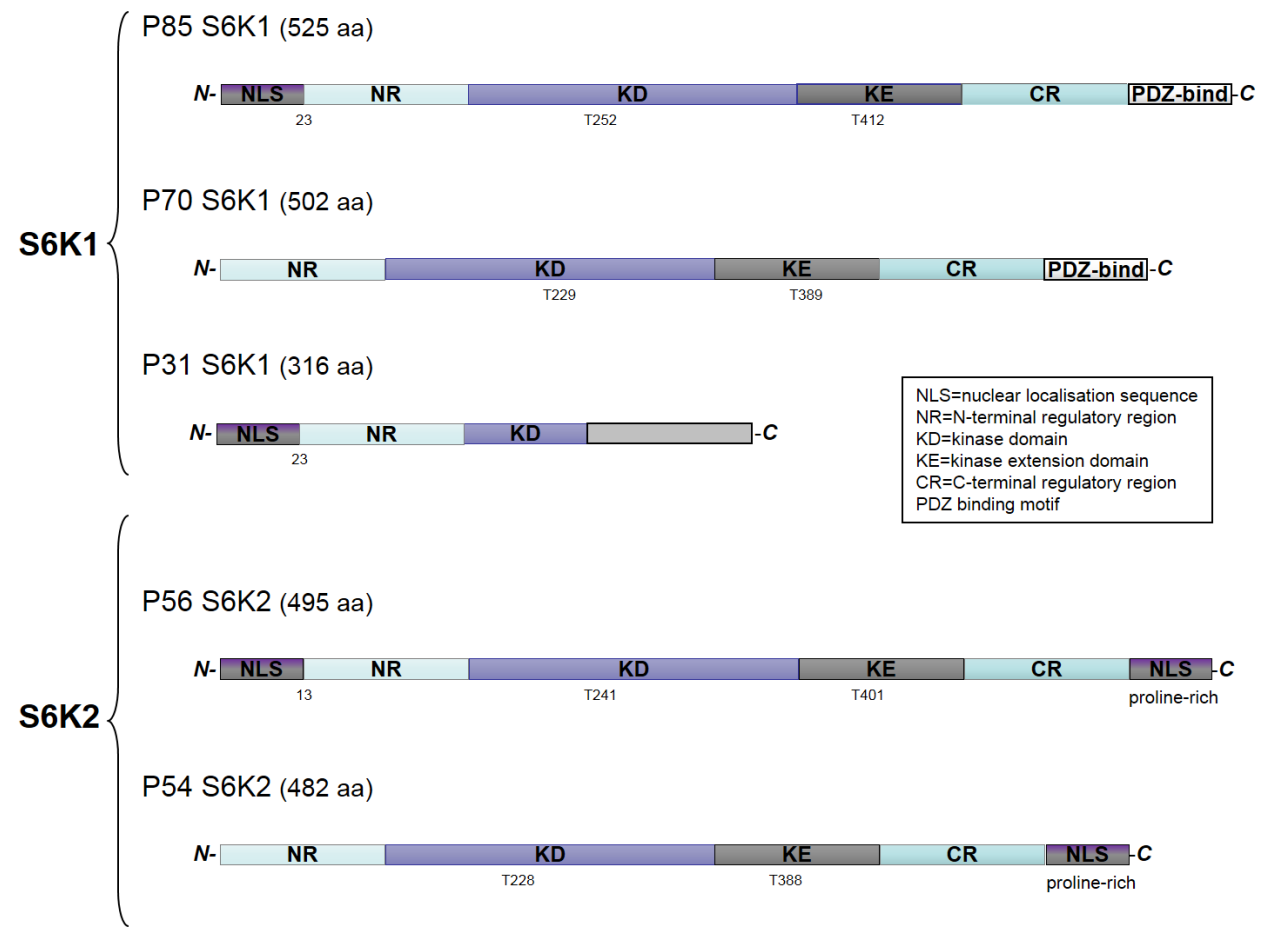

Figure 11 Domain structures and motifs of the known S6K1 and S6K2 isoforms. 
S6K1 and S6K2 share $70 \%$ overall amino acid identity, whereas the catalytic domains have even higher sequence homology with $>83 \%$ overlapping residues. Also the kinase extension and pseudosubstrate domains are very similar. Regarding S6K1, p70 is the predominant form, which is expressed ubiquitously among different tissues, localised mainly in the cytoplasm. The $\mathrm{p} 85$ isoform, resulting from an alternative translational starting site, contains an additional 23 amino acid nuclear localisation sequence (NLS), suggesting targeting of this isoform mainly to the nucleus [115]. Recently, a novel S6K1 isoform, p31S6K1 was discovered. The p31S6K1 contains a severely truncated kinase domain, nonetheless, it has been shown to possess transforming activity, which is probably kinase-independent [136]. Also S6K2 can be translated from alternative starting sites, and two different isoforms have been detected, termed p54 and p56S6K2, where p54 is the predominant form. Both S6K2 isoforms contain a C-terminal NLS, mainly localising them to the nucleus, and p56S6K2 contains an additional 13 amino acid N-terminal NLS [115].

Differences between S6K1 and S6K2 are mainly found in the N and C-terminal domains, which are probably important for localisation, regulation and function of the proteins. S6K1 contains a Cterminal PDZ binding domain, facilitating recruitment of S6K1 to the cytoskeleton via neurabin [137]. $\mathrm{S} 6 \mathrm{~K} 2$, in turns, harbours a C-terminal proline-rich domain allowing interactions with proteins containing SH3 or WW domains [138]. The domain structure and the several phosphorylation sites are well conserved and are found in the corresponding drosophila dS6K, indicating that the two S6K isoforms present in mammals result from gene duplication [139, 140]. Phylogenetic studies have shown that S6K1 likely is the ancestral kinase and that S6K2 has arisen at a later time [141].

Recently, the crystal structure of the S6K1 kinase domain was reported [142, 143]. Activated S6K1, like all known AGC kinases, show a more ordered structure than its inactive state, resulting from a salt bridge between Glu-143 and Lys-123 in the ATP-binding pocket. Characterisation of both the phosphorylated and non-phosphorylated states gives further insights in conformations important for S6K1 activation, and gives the opportunity for design of drugs targeting S6K. Shortly after the release of the 3D structure, the first specific S6K1 inhibitor PF-4708671 was developed [144].

\section{Regulation of $\mathrm{S} 6$ kinase function through PDK1/mTORC1 phosphorylation and PP2A dephosphorylation}

Soon after its discovery, it was shown that the activity of S6K could be inhibited by the immunosuppressor and antitumour agent Rapamycin [145-147], concluding mTOR as the major regulator of S6K function [137, 148] (Figure 10). mTORC1 phosphorylates and activates the S6 kinases at the conserved hydrophobic motif. Raptor, in the mTORC1 complex, binds directly to the TOR signalling (TOS) motif in the N-terminal part of S6Ks, mediating interaction between mTOR and S6K [115]. The full kinase activity of S6K1 and S6K2 also requires phosphorylations at other residues. Phosphorylation of the activation loop residue by PDK1 is preceded by several phosphorylations in the C-terminal region. Responsible kinases in vivo have not been fully elucidated, though several kinases have been suggested in vitro, among others ERK1/2, JNK1/2 and CDK1 [115]. Although several phosphorylation sites are conserved between S6K1 and S6K2 (Figure 11), some differences in the regulation are reported.

Regarding S6K1, a model was recently proposed where in a first step, the interaction between the Cterminal autoinhibitory domain and the $\mathrm{N}$-terminal is released by a $\mathrm{Ca}^{2+}$-dependent mechanism. This allows phosphorylation of S411, S418, T421 and S424 by mTORC1, which gives access to further 
phosphorylation of S371 and T389 by the same kinase. Finally, PDK1 can phosphorylate T229 in the activation loop, leading to an open conformation of S6K [139].

In S6K2, exchanging the mTORC1 target residue T388 to Glutamate (mimicking phosphorylation) renders a constitutively active kinase, insensitive to rapamycin and wortmannin, which is not the case for S6K1. However, probably phosphorylation of T388 is not enough for full activation, since T388 is not phosphorylated in S370 mutants, suggesting that phosphorylation of this site need to precede T388 phosphorylation. A model of S6K2 activation was earlier suggested where MEK dependent pathways phosphorylate Serine residues in the C-terminal domain, followed by S370 phosphorylation and mTORC1-dependent phosphorylation of T388. Phosphorylation of T388 in turn, allows phosphorylation of T228 by PDK1 [149].

Dephosphorylation and inactivation of $\mathrm{S} 6 \mathrm{~K} 1 / 2$ is mediated primarily by the protein phosphatase PP2A, in response to rapamycin or cellular stresses [115].

\section{Subcellular localisation and expression levels of the S6 kinases may determine their activity}

Posttranslational modifications of the S6 kinases, as well as their domain structures and interactions, allow regulation of their activity and subcellular localisation. P85 S6K1 is shown to be mainly localised to the nucleus, whereas P70 S6K1 is preferably seen in the cytoplasm, though it can be shuttled between the nucleus and the cytoplasm, despite not having an NLS. However, phosphorylation of Serine 17 by CK2, enhances its nuclear export back to the cytoplasm, ensuring a cytoplasmic fraction always to be present [150]. A recent study has shown, however, that in primary fibroblasts, p85 is cytoplasmic, p31 is exclusively nuclear and p70 can be shuttled between the nucleus and cytoplasm upon mTORC1 phosphorylation at T389. The nuclear-cytoplasmic shuttling of p70S6K1 is not dependent on its kinase activity [151]. As mentioned above, p56S6K2 contains two NLS motifs and is thus mainly found in the nucleus. The predominant S6K2 isoform p54S6K2 is shuttled between cytoplasm and nucleus, and in response to growth factors, phosphorylation of S473 in the C-terminal domain by PKC inactivates the NLS, rendering S6K2 in the cytoplasm.

The cellular functions of S6Ks are also due to the overall expression levels of the protein. The mechanisms regulating ubiquitination and proteasomal degradation of S6Ks are not well known, however it is shown that Roc1 ubiquitin ligase interacts with S6K1, but not S6K2, suggesting a further mechanism for different regulation of the kinases [152]. In addition, acetylation of Lysine residues of S6K2 seems to stabilise the protein, suggesting that this may opposite ubiquitination, in a similar mechanism as for p53 [153]. MDM2 has been shown to interact with S6K1 and S6K2 and induce its ubiquitination in response to serum and growth factors. Ubiquitination seems to be independent of phosphorylation and activation of the kinases [154]. 


\section{Discovery of the 4EBPs}

The rate limiting step in the translational apparatus is the binding of mRNA to the ribosomes, which is mediated by eukaryotic translation initiation factor $4 \mathrm{E}$ (eIF4E), directing the ribosomes to the 5 'cap structure of mRNA. The EIF4E gene, located at 4q23, was first cloned in 1987 [155] and eIF4E is a part of the large eIF4F complex, also containing eIF4A and eIF4G. The activity of eIF4E is considered mainly regulated by the $4 \mathrm{E}$ binding proteins (4EBPs). 4EBP1 and its close homologue 4EBP2 were initially cloned in 1994 and identified as interactors of eIF4E [156]. Later, these were mapped to the chromosomal regions 8p12 and 10q22, respectively [157]. In 1998, a third homologous eIF4E interacting protein named 4EBP3 was detected [158], and mapped to chromosome 5q31 [159]. The 4EBP1 protein is a rather small protein with 118 amino acids, encoded by three exons. The 120 amino acid sequence of 4EBP2 is $56 \%$ homologous with 4EBP1, also encoded by three exons, whereas 4EBP3, 57\% and $59 \%$ homologous to 4EBP1 and 4EBP2, respectively, is composed of 100 amino acids where the third exon is non-coding. 4EBP1 is the best characterised 4EBP [160]. The protein contains six known Serine/Threonine phosphorylation sites, whereof two are directly phosphorylated by mTORC1. 4EBP1 is foremost expressed in tissues involved in glucose and lipid homeostasis, mainly pancreas, muscle, liver and adipose tissue, whereas 4EBP2 is ubiquitously expressed. 4EBP3 lacks an N-terminal regulatory motif necessary for phosphorylation by mTORC1, and is assumed to possess different functions from 4EBP1 and 4EBP2.

\section{S6Ks and 4EBP1 in regulation of protein synthesis}

In higher eukaryotes, pre-mRNAs are commonly spliced, removing introns before translocation to the cytoplasm. During splicing, several protein complexes are bound to the mRNA, mainly TREX, CBP80/20 and the exon junction complex (EJC). The protein complexes facilitate nuclear export of mRNA and are involved in a pioneer round of translation, where mRNAs with premature termination codons are eliminated. During this step, EJCs dissociate from the complex and CBP80/20 is exchanged to eIF4E, facilitating recruiting of a translational initiation complex (Figure 12). EIF4E recruits eIF3 and the 40S ribosomal subunit to $5^{\prime}$-mRNA. mTORC1 binds eIF3 in the complex and phosphorylates 4EBP1 and S6K1. Phosphorylation of 4EBP1 leads to its dissociation from eIF4E, promoting recruiting of proteins to the complex, including the scaffolding protein eIF4G, the RNA helicase eIF4A and PAPB1. Activated S6K1 phosphorylates rpS6 in the 40S ribosomal subunit, and also the eIF4A cofactor eIF4B, recruiting it to eIF4A [125]. S6K1 also phosphorylates and promotes degradation of PDCD4, a tumour suppressor that binds and inhibits eIF4A. S6K1 also increases the rate of translation by phosphorylating eEF2K. The eEF2K kinase is a negative regulator of translational elongation by phosphorylation and inactivation of eEF2. S6K1 phosphorylation of eEF2K at S366 inactivates the kinase, promoting translation [161] (Figure 12). Altogether, these steps lead to activation of eIF4A helicase, facilitating 40S scanning for the start codon and translational initiation. 
a)

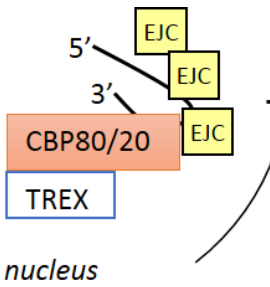

During splicing, mRNA is imprinted with protein complexes.

$E J C=e x o n$ junction complex

c)

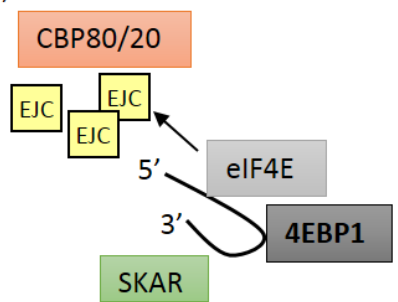

Pioneer round of translation

*SKAR binds CBP80-bound mRNA-complex.

*EJC dissocates from mRNA

${ }^{*} \mathrm{CBP} 80 / 20$ is exchanged to elF4E-4EBP1

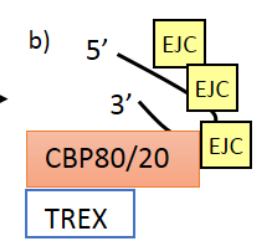

cytoplasm

The complex is translocated to the cytoplasm

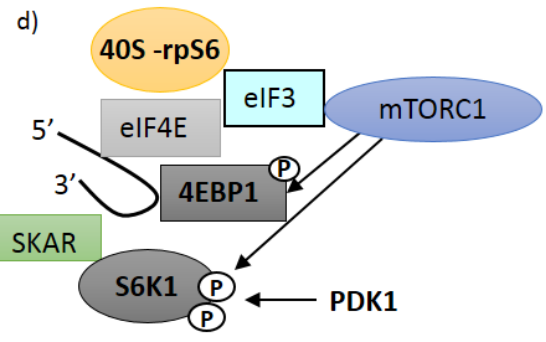

Translational initiation

* eIF4E recruits eIF3 and the $40 \mathrm{~S}$ ribosomal subunit.

*SKAR facilitates S6K1 interaction with the complex.

*mTORC1 binds to eif3 in the complex and phosphorylates 4EBP1 and S6K1. -4EBP1 dissociates, activating eIF4E

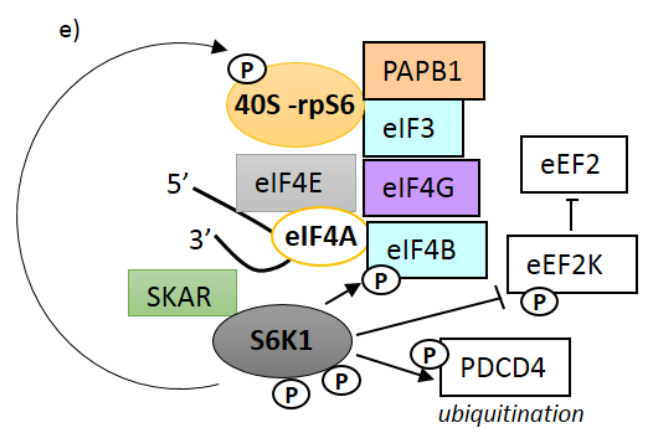

Activated EIF4E recruits -elF4G (scaffolding protein) -elF4A (RNA-helicase)

-PAPB1 (polyA-binding protein)

Activated S6K1 phosphorylates -rpS6 -elF4B, recruits to elF4A

-PDCD4, leading to its degradation, activating elF4A -eEF2K, leading to its inhibition, activating eEF2

=>elF4A starts unwinding mRNA, promoting ribosomal $40 S$ scanning after the start codon.

Figure 12 Roles of S6K1 and 4EBP1 in the translational machinery [121, 124, 125]. 
mTOR/S6K1 has been shown to facilitate translation of spliced mRNA over unspliced, in a process dependent of the Aly/REF family homologue SKAR. Activated nuclear S6K1 is recruited to newly synthesised mRNA by SKAR at the exon-junction complex during splicing. Knockdown of S6K1, SKAR or EJC components leads to a diminished translation of spliced mRNA. SKAR is phosphorylated by S6K1, but not S6K2, and knockdown of S6K2 has no impact on the translational rate of spliced mRNA, suggesting that this function is isoforms specific [124].

Most studies regarding S6K in regulation of protein synthesis is restricted to S6K1. However, also S6K2 has been shown involved in the translational machinery, and may possess functions independent of S6K1. In vitro, FGF2 activation results in increased levels of S6K2 [162], and formation of a complex involving S6K2, B-raf and PKCE. PKC $\varepsilon$ activates S6K2 by phosphorylation, leading to upregulation of synthesis of antiapoptotic proteins; a mechanism which is probably involved in chemoresistance [117, 162].

RpS6 is the most well-known substrate for S6 kinases and its phosphorylations have been investigated thoroughly [125]. The five phosphorylation sites are situated in a well conserved region in the Cterminal domain and are suggested to be phosphorylated in the ordered manner, S236 $>$ S235 $>$ S240 $>$ S244 $>$ S247. S236 is the primary substrate and the only residue within a consensus recognition sequence of S6K. Knockout studies in mice have shown that the levels of phosphorylated rpS6 are significantly reduced when S6K2 is deleted, whereas the levels are almost restored in S6K1knockout mice, indicating that S6K2 may be the primary S6 kinase [163]. However, knockout of the rpS6 phosphorylation sites result in a phenotype similar to that of S6K1-/-, suggesting that S6K1 may be essential for specific pools of rpS6, e.g. during different times of the development or in different cellular compartments [164]. Phosphorylated rpS6 has been detected both in the nucleus and the cytoplasm of the cells [163]. The rpS6 protein is conserved and can be found in yeast, however yeast does not possess any homologue to S6K1 and S6K2 [125].

RpS6 is localised to the interface between the 60S and 40S ribosomal subunits where it interacts with tRNA, mRNA and translational initiation factors, suggesting a key role in regulation of protein synthesis [125]. However, studies regarding the relationship between rpS6 phosphorylation and efficacy of protein synthesis have resulted in contradictory findings. Knock-in of $\mathrm{rpS}^{\mathrm{P}-/-}$ in mice has shown no difference in the proportion of ribosomes engaged in polysomes in liver cells and the rate of global protein synthesis increases in mouse embryonic fibroblasts (MEFs) from $\mathrm{pS}^{\mathrm{P}-/ \mathrm{-}}$ mice [164]. Thus, rpS6 phosphorylation may in some cell types result in downregulation of protein synthesis, suggesting S6Ks to be responsible for fine tuning of protein synthesis [125]. Early studies of rpS6 phosphorylation have demonstrated a strong correlation between S6K expression, rpS6 phosphorylation and the translation of 5 'terminal oligopyrimidine tract (TOP) mRNA, encoding many components of the translational machinery. However, subsequent studies have failed to show this relationship [125], and the theory was more or less discarded when it was shown that S6K1-/S6K2mice exhibited complete 5'TOP mRNA translation [163]. Though, the translational efficacy of 5'TOP mRNAs has in some cell types been shown rapamycin dependent, and a role for the mTOR/S6K pathway in 5'TOP mRNA translation cannot be totally ruled out [125]. 
Nuclear substrates of S6K1 and S6K2: involvement in transcriptional regulation and cell cycle control

Studies have shown that S6K1 has nuclear substrates, suggesting a role in regulation of transcription, in addition to its functions in translation. One nuclear S6K1 target is the transcription factor CREM $\tau$. CREM $\tau$ modulates gene expression by binding to cAMP-responsive elements, commonly activated by the adenylate cyclase signalling pathway. The role of S6K1 phosphorylation of CREM $\tau$ for gene expression is still unknown [125]. ER $\alpha$ was recently shown to be a substrate of S6K1 [165]. S6K1 phosphorylates ER at S167, leading to transcriptional activation and subsequent increased cell proliferation, probably independent of ligand binding. In a recent study, phosphoproteomic screening identified four new nuclear S6K1 substrates in vitro; GRP75, CCT $\beta$, PGK1 and RACK1. GRP75 and CCT $\beta$ are involved in chaperone function, whereas PGK1 is an enzyme in the glycolytic pathway and RACK1 is a scaffold protein involved in vesicle trafficking [166] (Figure 10).

Whether S6K2 is involved in regulation of transcription remains unknown. However, SH3-binding motifs, which can be found in S6K2, are shown to facilitate interaction of cofactors with ER $\alpha$ [138], allowing to speculate that S6K2 also has the potential to act as a cofactor of the receptor. Nuclear S6K2, but not S6K1 has been shown to associate with several RNA-binding proteins, including the hnRNPs [120]. HnRNPs are chromatin-associated nuclear proteins that bind specifically to different RNA sequences and are involved in several steps of RNA processing, including splicing, polyadenylation, mRNA stability and translational regulation [167]. Upon serum stimulation, mTOR associates with the hnRNP-S6K2 complex and activates S6K2 which promotes cell proliferation through unknown mechanisms [120]. Recently, it was also shown that S6K2, but not S6K1, can bind to the transcription factor YY1 in serum stimulated cells [116] (Figure 10).

The S6 kinases have also been implicated in cytoskeletal dynamics in the context of cell cycle regulation. In vitro, both $\mathrm{S} 6 \mathrm{~K} 1$ and $\mathrm{S} 6 \mathrm{~K} 2$ have been shown active throughout the cell cycle, where S6K1 peaks in the mitotic (M) phase and S6K2 in both gap2 (G2) and M [168]. However, in mid G1 phase p70S6K1 becomes phosphorylated by mTOR at T389 and translocated to the nucleus [169]. S6K2, but not S6K1, has been reported to localise to the centrosome during all steps of the cell cycle, suggesting that it may be involved in cytoskeletal regulation through centrosome signalling [170]. S6K has also been shown to regulate the cell cycle by activating Myc function, through phosphorylation and inactivation of the MYC suppressor Mad [171]. mTORC1/S6K1 may also stimulate cellular proliferation by upregulating pyrimidine synthesis $[172,173]$.

\section{The S6 kinases in regulation of cell growth: implications from knock-out studies}

The expression of the S6 kinases in different mouse and human tissues has recently been investigated [141]. A similar mRNA expression of both the kinases could be detected in all tissues examined. The levels of S6K1 protein were uniform in all tissues, whereas S6K2 varied profoundly, suggesting that the levels of S6K2 can be regulated at posttranscriptional level in a tissue-specific manner. Specifically, in the adrenal gland, S6K2 protein levels were found very low, whereas the expression was totally absent in the kidney.

The S6 kinases are believed to have overlapping functions, however there are also data indicating divergence in their biological activities. Knock-out of $S 6 K 1$ in mice, and $d S 6 K$ in drosophila have 
resulted in a reduction in animal body size during embryogenesis, as a result of a decrease in individual cell size [140,174]. In contrast, $S 6 K 2^{-/}$mice had normal or slightly increased body size. Of note however, S6K1-deficient mice showed a significant upregulation of S6K2 protein in several tissues, suggesting a compensatory mechanism, which may explain why the phenotype of size reduction was mostly overcome by adulthood [163]. Deletion of both $S 6 K 1$ and $S 6 K 2$ in mice, as well as $d S 6 K$ in drosophila have been shown semilethal, severely reducing the viability. In contrast, no differences in lethality of S6K1 or S6K2 deficient mice have been seen, supporting compensatory and essential roles for the kinases in normal development [163,174]. The reduced viability of S6K deficient mice and drosophila may partly be explained by its ability to phosphorylate and inactivate the proapoptotic factor BAD [175].

In addition to a reduction of animal body size, S6K1 knockout mice exhibited a phenotype comparable to type 2 diabetes mellitus, with hypoinsulinemia and glucose intolerance, due to a selective decrease in size of the pancreatic $\beta$-cells [176]. It was also shown that the mice were resistant to developing diet-induced obesity, as a result of inactivation of a negative feedback-loop, where chronic exposure to nutrients causes desensitisation of the insulin receptor [177].

\section{Activation of the PI3K/AKT/mTOR pathway as a clinical marker and target in cancer therapeutics}

Deregulated metabolic pathways as a result of the western lifestyle with excess energy intake is suggested to be predisposing for cancer as well as other well-fare diseases such as diabetes. Humans carrying mutations in GHR leading to reduced IGF production and subsequent mTOR signalling are resistant to development of type II diabetes and malignant tumours, implicating an important connection between the origin of these diseases, where this pathway may be a major hub [94]. The $\mathrm{PI} 3 \mathrm{~K} / \mathrm{AKT} / \mathrm{mTOR}$ pathway is upregulated in $>70 \%$ of breast cancers. Aberrant activation of RTK signalling is common, mainly through increased autocrine ligand activation, receptor amplification/overexpression, chromosomal translocations or mutations producing a constitutively active kinase. As mentioned previously, ERBB2 at $17 \mathrm{q} 23$ is amplified and overexpressed in approximately $15 \%$ of breast cancers. Also, FGFR1 at 8p12 is found amplified and overexpressed in about $10 \%$ of cases. Furthermore, commonly appearing aberrations are PIK3CA mutations, $A K T$ gene amplification/mutations as well as overexpression and activation of several upstream growth factors and RTKs. Loss of function of negative mTOR regulators e.g. PTEN, TSC1/2 and LKB1 is also frequently found in breast tumours [178] (Table 3). The most common aberration is mutations in PIK3CA, foremost in the helical domain (E542K or E545K) or the kinase domain (H1047R), leading to a constitutively active kinase. The highest frequency of PIK3CA mutations are found in the ER positive subgroup [178]. 
Table 3 Common alterations in the PI3K/AKT/mTOR pathway in breast cancer [60, 61, 178, 179].

Frequency of alterations

\begin{tabular}{|c|c|c|c|c|}
\hline Factor & Alteration & Luminal (ER+) & HER2+ & $\begin{array}{l}\text { Basal } \\
\text { (Triple Negative) }\end{array}$ \\
\hline ERBB2 (HER2) & $\begin{array}{l}\text { Amplification or } \\
\text { overexpression }\end{array}$ & $10 \%$ & $\sim 100 \%$ & $0 \%$ \\
\hline$E G F R$ & Amplification & \multicolumn{3}{|c|}{$0.8 \%$ of all cases } \\
\hline$I G F 1 R$ and INSR & $\begin{array}{l}\text { Receptor activation, } I G F 1 R \\
\text { amplification }\end{array}$ & $41-48 \%$ & $18-64 \%$ & $42 \%$ \\
\hline FGFR1 & $\begin{array}{l}\text { Amplification, activating } \\
\text { mutation }\end{array}$ & $8.6-11.6 \%$ & $5.4 \%$ & $5.6 \%$ \\
\hline PTEN & $\begin{array}{l}\text { Loss-of-function mutation } \\
\text { or reduced expression }\end{array}$ & $29-44 \%$ & $22 \%$ & $67 \%$ \\
\hline $\begin{array}{l}P I K 3 C A \\
(p 110 \alpha / P I 3 K)\end{array}$ & Activating mutation & $28-47 \%$ & $23-33 \%$ & $8-25 \%$ \\
\hline $\begin{array}{l}P I K 3 C B \\
(p 110 \beta / P I 3 K)\end{array}$ & Amplification & \multicolumn{3}{|c|}{$5 \%$ of all cases } \\
\hline $\begin{array}{l}\text { PIK3R1 } \\
(p 85 \alpha / P I 3 K)\end{array}$ & Inactivating mutation & \multicolumn{3}{|c|}{$2 \%$ of all cases } \\
\hline$K R A S$ & Activating mutation & \multicolumn{3}{|c|}{$4-6 \%$ of all cases } \\
\hline$I N P P 4 B$ & $\begin{array}{l}\text { Reduced expression or } \\
\text { genomic loss }\end{array}$ & $10-33 \%$ & $54 \%$ & $53 \%$ \\
\hline$L K B 1$ & $\begin{array}{l}\text { Loss-of-function mutation } \\
\text { or reduced expression }\end{array}$ & & $?$ & \\
\hline$T S C 1 / 2$ & $\begin{array}{l}\text { Loss-of-function mutation } \\
\text { or reduced expression }\end{array}$ & & $?$ & \\
\hline$P D K 1$ & $\begin{array}{l}\text { Amplification or } \\
\text { overexpression }\end{array}$ & $22 \%$ & $22 \%$ & $38 \%$ \\
\hline$A K T 1$ & Activating mutation & $2.6-3.8 \%$ & $0 \%$ & $0 \%$ \\
\hline$A K T 2$ & Amplification & \multicolumn{3}{|c|}{$2.8 \%$ of all cases } \\
\hline RPS6KB1 & $\begin{array}{l}\text { Amplification or } \\
\text { overexpression }\end{array}$ & \multicolumn{3}{|c|}{$3.8-12.5 \%$ of all cases } \\
\hline
\end{tabular}


Evidence for involvement of S6K kinases in cancer development are increasing. RPS6KB1 amplification and $\mathrm{S} 6 \mathrm{~K} 1$ protein overexpression has earlier been associated with a worse outcome in breast cancer [60-63, 180]. Since S6K2 was discovered much later than S6K1, very little is yet known about its oncogenic properties. However, S6K2 overexpression in breast cancer cells has been reported [181]. Strong accumulations of S6K2 in the nuclei of breast cancer cells have been detected in $80 \%$ of tumours, whereas the same result for S6K1 was $25 \%$. The increase in S6K2 expression is mainly found in the nuclei of cancer cells in peripheral areas of the tumours. In addition, the accumulation of S6K2 was correlated with expression of the proliferation markers PCNA and Ki-67. $R P S 6 K B 2$, the gene encoding $\mathrm{S} 6 \mathrm{~K} 2$ is located very adjacent to the $C C N D 1$ gene, in the chromosomal region $11 \mathrm{q} 13$, which is frequently amplified in many breast cancers.

A hallmark of cancer is the ability of cells to leave the primary tumour site and migrate to distant areas where space and nutrients are more accessible [20,21]. Recent studies have indicated that the S6 kinases may have a role in this process. In ovarian cancer cells, it was shown that S6K1 can act as an actin filament cross-linking protein and also activate Rac1, cdc42 and their downstream effector PAK1, promoting cytoskeletal reorganisation and migration [182]. S6K1 has also been shown able to upregulate mRNA expression of the embryonic transcription factor SNAIL in ovarian cancer cells, inducing morphologic changes consistent with EMT characteristics e.g. downregulation of E-cadherin [183].

4EBP1 is generally considered as a tumour suppressor, due to the ability of unphosphorylated protein to inhibit the translational machinery, upon negative regulation by mTOR. However, several findings point towards a dual role for 4EBP1, possessing both tumour suppressive and oncogenic functions. Tumour suppressor genes are commonly deleted in cancer, though the gene encoding 4EBP1 is located in the chromosomal region $8 \mathrm{p} 12$, which is frequently amplified in breast cancer and several studies show a high correlation between 4EBP1 amplification and increased mRNA expression [184187]. In addition to gene amplification, another suggested mechanism of 4EBP1 overexpression is through Myc dependent transcription. Amplification or induced expression of Myc have been shown to promote Myc binding to the 4EBP1 gene and increase its expression. This in turn leads to inhibition of autophagy and rapamycin resistance [188].

High levels of phosphorylated 4EBP1 have been associated with a high grade and worse outcome in several malignancies $[189,190]$, which is generally considered as a result of its role as a marker of activated mTOR signalling. However, in large tumours, overexpression of unphosphorylated 4EBP1, and eIF4G in response to hypoxia has been shown to switch the ribosomal machinery from capdependent to cap-independent translation [191]. This enables tumour cells to increase selective translation of mRNAs containing internal ribosome entry sites (IRESs), including VEGF, promoting angiogenesis. 4EBP1 has also recently been implicated in a positive feedback loop by binding and stabilising mTORC1, thereby promoting its activation [192]. The dual role of 4EBP1 in cancer may be explained by its emerging divergent roles in different cellular compartments. Approximately $30 \%$ of the 4EBP1 expressed in cells is located in the nucleus, where it has a role in regulating the availability of eIF4E for the cytoplasmic translational machinery, by retaining eIF4E in the nucleus [193]. High nuclear levels of 4EBP1 would thus inhibit translation and subsequent proliferation. 
Epidemiologic studies have shown that obesity and type 2 diabetes are risk factors for the development of several cancer types, including breast cancer [194]. In addition, breast cancer patients with underlying type 2 diabetes are shown to have more advanced breast cancers and a poorer prognosis compared to patients without diabetes [195, 196]. A complex interplay between insulin, the IGF system, and glucose metabolism in different tissues and during different time points may underlie the connection between diabetes and cancer. Under normal circumstances, rising blood glucose levels following a meal triggers insulin release from beta-cells of the pancreas (Figure 13). In addition, GH release from the pituitary stimulates IGF1 release from the liver. Insulin acts on most tissues, though mainly liver, muscle and adipose tissues. In muscle and adipose tissue insulin stimulates glucose uptake by controlling GLUT4 glucose transporters through the PI3K/AKT pathway. IGF1 is, as previously mentioned, a master regulator of whole body cellular growth. Type 2 diabetes is generally defined by decreased insulin sensitivity, mainly in liver, muscle and adipose tissues [197]. This is suggested to result from among others downregulation of $\mathrm{PI} 3 \mathrm{~K} / \mathrm{AKT} / \mathrm{mTOR}$ signalling in these tissues. In response to enhanced blood glucose levels, pancreatic release of insulin increases to compensate for the insulin resistance. Eventually the ability of the beta-cells to produce insulin often fails to compensate the insulin resistance, with hyperglycaemia as a result, and type 2 diabetes can be diagnosed [198, 199].

Hyperinsulinemia may stimulate cancerous development and growth directly by acting on epithelial tissues that commonly are not insulin resistant. Indirectly, rising insulin levels in turn lead to increased levels of free circulating IGF1 as well as oestrogens, by influencing the expression of binding proteins, e.g. globulins. Also hyperglycaemia has been associated with increased risk of developing cancer, among others breast cancer [200], as well as a poor breast cancer outcome [201].

IGF-signalling may play divergent roles in different stages of mammary gland development, and could therefore also have different impact on breast cancer susceptibility in different ages [202]. In the mammary gland from prepubertal mice, IGF1 was shown to promote proliferation mainly through $\mathrm{PI} 3 \mathrm{~K} / \mathrm{AKT} / \mathrm{mTOR}$-signalling increasing the cyclin D1 protein levels. In the postpubertal gland, IGF1 instead promoted proliferation through ERK/MAPK signalling, independent on cyclin D1, although still via upregulation of mTOR. ER-levels are suggested to determine the proliferative route of IGF1. The levels of ER change through development stages, and are high in prepubertal but lowered in postpubertal stages as a result of increased levels of circulating oestrogens. In the postmenopausal gland, ER levels are again elevated, which in combination with high levels of circulating IGF1 as a result of dietary factors is a potential risk factor for breast cancer in this group. However, IGF1 levels in the prepubertal stage where ER-levels are also high may indeed be a predisposing factor for breast cancer development. This is supported by the fact that young African American girls often develop early onset, aggressive, breast cancer and also have higher levels of circulating IGF1, in comparison with European girls in the same ages.

As a consequence of the importance of the insulin/IGF1 axis for both diabetes and cancer, therapies aiming to maintain the energy homeostatic balance in diabetes may also be promising in the prevention and treatment of cancer. To date, the biguanide metformin is the most widely prescribed drug to treat hyperglycaemia in diabetes [203]. Several prospective studies have found an association between metformin use in diabetic patients and a decreased risk of cancer [204, 205], as well as a favourable outcome among cancer patients [206]. Metformin was clinically introduced already in the 1950 s as an antihyperglycaemic agent, however, the exact cellular and molecular mechanisms of 
metformin action are yet to be elucidated. Physiologically, metformin has shown favourable effects on several aspects of type 2 diabetes, leading to stabilised levels of blood glucose, reduced levels of circulating insulin and IGF1, as well as increased insulin sensitivity in peripheral tissue (reviewed in $[203,207]$. It has been suggested that many of these effects are primarily mediated through an increased LKB1/AMPK activity, resulting from metformin-induced increase in AMP/ATP ratio [207]. Recent data have demonstrated that metformin may act on hepatocytes by specifically and mildly inhibit mitochondrial respiratory chain complex I, leading to reduced gluconeogenesis [203]. The anti-cancer effects of metformin are suggested to result from lowered blood glucose, insulin and IGF1, and also by direct inhibition of tumour growth and proliferation mediated by LKB1/AMPK activation in turn inhibiting mTOR signalling [203, 208]. 


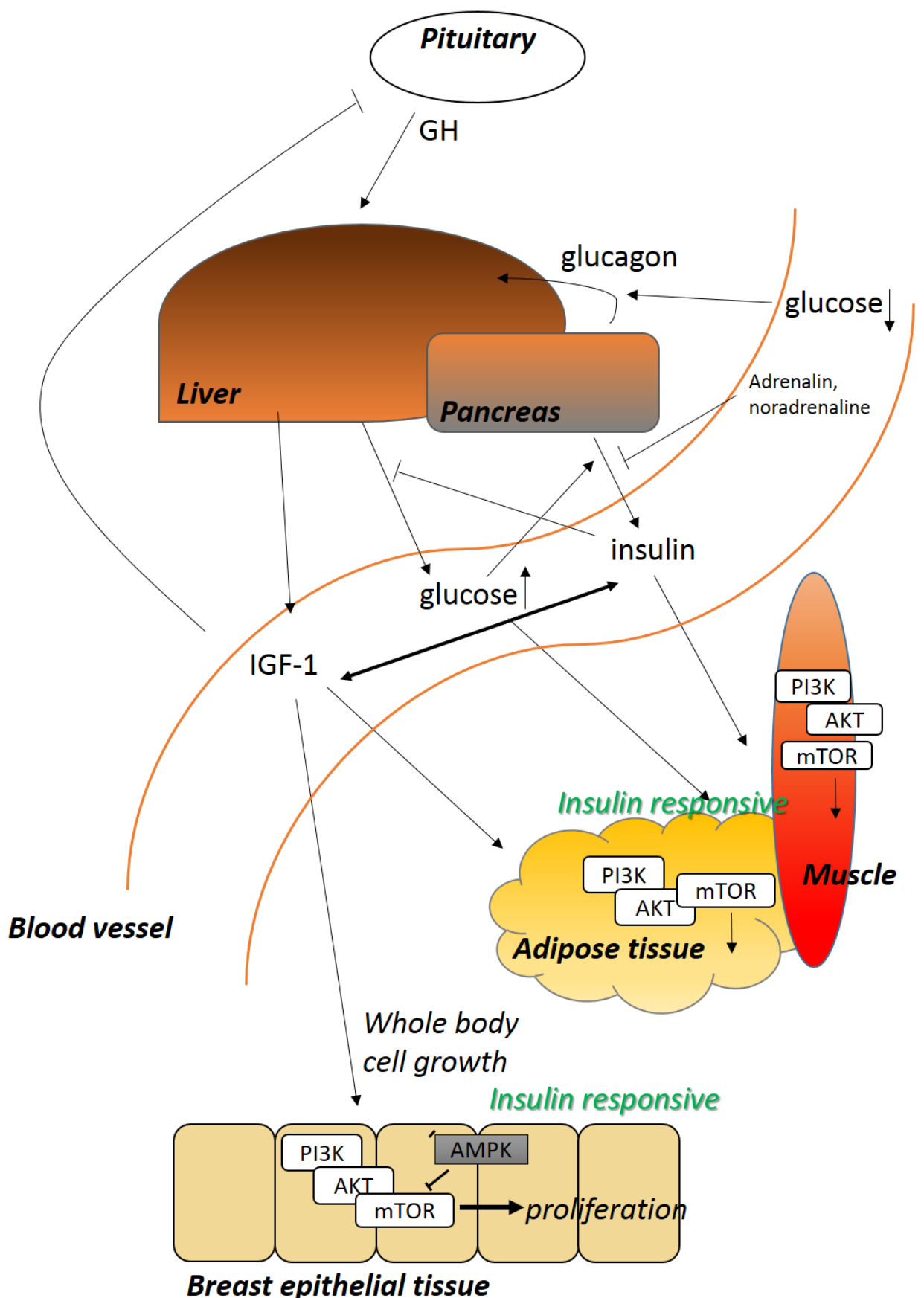

Figure 13 Insulin/IGF signalling and its effects on mammary gland epithelial proliferation under normal circumstances. 


\section{$\mathrm{PI3K} / \mathrm{AKT} / \mathrm{mTOR}$ and its effectors as potential targets in cancer therapy- feedback regulation a bottle neck}

Upregulation of the mTOR signalling pathway facilitates several hallmarks of cancer. Interplay between ER and PI3K/AKT/mTOR signalling is suggested to promote cellular growth and proliferation, and also diminish the effects of ER targeted therapies. The cross-talk between ER and growth-signalling pathways can occur on multiple levels in a bidirectional manner. Several RTKs, as well as ligands and adaptor proteins are transcriptional targets of the ER. In addition, ER can rapidly activate PI3K/AKT through non-genomic mechanisms. AKT, S6K1 as well as ERK are able to activate ER in a ligand-independent manner by phosphorylating the receptor at $\mathrm{S} 167$ [165, 209, 210]. Also, AKT/mTOR regulates translation, as well as transcription of the ER transcriptional targets Myc and cyclin D1, promoting cell cycle progression [211]. Endocrine resistant cells show upregulation of IGF1R, IR, HER2, EGFR, FGFR1 and subsequent PI3K/AKT/mTOR activation [13], and activation of the pathway has been shown a predictive marker for poor response to endocrine therapies [212217]. Concomitant targeting of ER and HER 2 is used clinically for tumours expressing both receptors. In vitro, combining ER targeted therapies with IGF1R inhibitors has been shown useful [213], but dual targeting in clinical phase III studies has so far failed to show any benefit ([218] and references therein). As mentioned above, the use of mTOR inhibitors in combination with aromatase inhibitors has been shown successful [7] and is today clinically approved. The clinical benefit of mTOR inhibitors, though, seems to vary between different tumour types and so far the effectiveness has mainly been restricted to disease stabilisation rather than tumour regression.

An arising problem when using $\mathrm{PI} 3 \mathrm{~K} / \mathrm{AKT} / \mathrm{mTOR}$ antagonists is the phenomenon of counteracting feedback mechanisms, where the most well-known involves S6K1 diminishing AKT signalling through inhibition of IRS1 and IRS2 (Figure 10) [125]. The IRS1/2 adaptor proteins activate $\mathrm{PI} 3 \mathrm{~K} / \mathrm{AKT}$ signalling upon stimulation of IGF and other growth factors through its receptors. Activated S6K1 phosphorylates IRS1 at S307, leading to dissociation from the receptor [219]. S6K1 can also phosphorylate IRS1 at S1101, blocking its phosphorylation and activation by the RTK [220]. S6K1 and S6K2 also seem to regulate the stability of IRS1 mRNA [219], and S6K1 is involved in a positive feedback mechanism by phosphorylating mTOR at T2446/S2448 [110, 111]. However, the effect of mTOR phosphorylation at these sites is not fully elucidated. The S6 kinases have also been shown to possess negative feedback by regulating the subcellular localisation of PTEN, a negative regulator of PI3K/AKT signalling [221]. PTEN is localised to the nucleus of differentiated and cell cycle arrested cells (G0/G1 phase), but is exported to the cytoplasm during G1-S transition, a localisation where it often can be found in tumour cells. S6K1/2 was able to bind PTEN and promote its nuclear export, facilitating cell cycle progression. Whereas $\mathrm{S} 6 \mathrm{~K} 1$ is involved in negative feedback loops of AKT/mTOR signalling, S6K2 has been shown to activate AKT and promote cell survival in MCF7 breast cancer cells [222]. This suggests that S6K1 and S6K2 may have different roles on AKT activation.

Studies have indicated additional roles of 4EBP1, independent of mTORC1. Rapalogs, mainly targeting mTORC1, have been shown to completely inhibit pS6K but only partially p4EBP1 [223]. In bladder cancer, 4EBP1 was shown to be regulated by PI3K, but not through mTORC1 [224] and mTOR-independent 4EBP1 phosphorylation has been associated with resistance to mTOR kinase inhibitors [225]. Additional kinases for 4EBP1 regulation remain to be identified, where upstream factors of the PI3K/AKT pathway are likely candidates. 
Overall, the mTOR pathway has emerged as a critical effector in the interplay between nutrients, growth factors and hormones regulating normal physiology, and consequently a key factor in the cellular deregulations occurring in cancerous states. Findings point towards significant differences in regulation and effects of its downstream factors S6K1 and S6K2 in normal physiology, which may also be relevant in the clinical setting. In addition, an oncogenic role of 4EBP1 is emerging. An increased knowledge of the intracellular pathways involved in the mTOR signalling circuit, and its role in different tissues and during different conditions and states, would be potentially helpful in the future prevention and treatment of cancer. 


\section{AIM OF THE PROJECT}

The purpose of this project was to evaluate the role of alterations downstream the mTOR signalling pathway for breast cancer development, prognosis and treatment response, specifically:

Study 1: Due to the location of S6K2 in the 11q13 chromosomal region commonly amplified in malignancies, and the high homology between S6K1 and S6K2, investigate whether S6K2 is amplified and overexpressed in breast cancer and evaluate its prognostic and endocrine treatment predictive role, in comparison with S6K1 amplification.

Study 2: Look further into the 11q13 amplicon and study S6K2 amplification in relation to other candidate oncogenes in the region as well as to genome wide copy number alterations, and to evaluate the $11 \mathrm{q} 13 / 8 \mathrm{p} 12$ coamplification and its prognostic value.

Study 3: Investigate the S6K2/4EBP1 (11q13/8p12) coamplification/coexpression identified in study 2 in larger cohorts, as well as the prognostic and endocrine treatment predictive role of 4EBP1 at the protein level.

Study 4: Investigate different roles of S6K1 and S6K2 and their individual potentials as new clinical targets in breast cancer. 


\section{MATERIALS AND METHODS USED IN THE STUDIES}

This part contains a brief description and comments on the different patient materials and methods used in the studies. Further details can be found in the respective publication.

\section{Breast cancer patient materials and models}

Study 1-3 encompass two cohorts from the randomised adjuvant Stockholm tamoxifen trials, referred to as Stockholm 2 and Stockholm 3. In Study 3 and 4, three publically available datasets were used to confirm some of the results. In addition, an in vitro model of breast cancer was used in Study 4. This section will describe the different materials used in the studies, discuss their original designs and clinical value.

\section{The treatment randomised Stockholm trial}

To determine the value of a potential new treatment, clinical procedure or biological marker, large randomised controlled trials are considered the golden standard [226, 227]. Randomisation serves the purpose to balance the groups to avoid selection biases and thereby factors confounding the results. It also permits the use of probability theory and subsequent statistical tests. For correct randomisation, no prior knowledge about assignment groups should be known that could influence participants or the researchers. Patient materials from large randomised clinical studies are rather rarely available, and therefore highly valuable.

The Stockholm 2 and Stockholm 3 cohorts used in the present study are derived from a large, randomised trial conducted by the Stockholm breast cancer group between November 1976 and April 1990, mainly aiming to evaluate the benefit from adjuvant tamoxifen treatment. All patients were randomised to receive tamoxifen for two years, or no endocrine treatment. Patients in the Stockholm 2 cohort were further randomised, using a $2 \times 2$ factorial study design, to postoperative radiotherapy (RT) or cyclophosphamide-methotrexate-5-fluorouracil (CMF)-based chemotherapy. Most of the patients in the tamoxifen arm, if disease-free after two years, were then randomised to receive tamoxifen for three more years, or no further adjuvant treatment. Detailed information about study designs and long-term follow-up data have been previously reported in detail [228, 229]. Patients in the Stockholm 2 cohort were postmenopausal and had positive lymph nodes and/or a tumour diameter exceeding $30 \mathrm{~mm}$, whereas the Stockholm 3 cohort consisted of postmenopausal breast cancer patients with a tumour diameter $\leq 30 \mathrm{~mm}$ and no lymph node involvement. Patient flow through the studies is presented in Figure 14 and clinicopathological data can be found in Table 4. Retrospective studies of biomarkers were approved by the local ethics board at the Karolinska Institute, Stockholm, Sweden, and at the time, no further consent from the patients was needed.

ER expression was determined at the time of diagnosis, before 1988 this was done using isoelectric focusing and after this year, quantitative enzyme immunoassay (EIA) was used [228, 229]. In the 
Stockholm 3 cohort, where tissue microarrays (TMAs) were available, ER and PgR status were further determined retrospectively by IHC [230]. Cut-off levels for ER and PgR positivity were $>10 \%$ stained nuclei or, when IHC data was not available, $0.05 \mathrm{fmol} / \mu \mathrm{g}$ DNA. Isoelectric focusing/EIA and IHC data have been shown to be comparable [231]. In the Stockholm 2 cohort, HER2 protein was quantified retrospectively by flow cytometry [232] and HER2 amplification was determined with quantitative Real-Time PCR [233]. HER2 protein expression in the Stockholm 3 cohort was evaluated with IHC as described elsewhere [234], whereas tumour grade was evaluated retrospectively according to the Nottingham system [230]. In the Stockholm 2 cohort, S-phase fraction (SPF) was previously determined by flow cytometry [235], and mutations in PIK3CA exon 9 or 20 was assessed by single-strand conformational polymorphism (SSCP) [179]. Breast cancer subtypes in Stockholm 3 were defined according to St Gallen 2011 [9] as follows: Luminal A (ER+ and/or PgR+, Her2-, grade I-II), Luminal B1 (ER+ and/or PR+, HER2-, grade III), Luminal B2 (ER+ and/or PR+, HER2+), HER2-like (ER- and PR- and HER2+), and Basal-like (ER- and PR- and HER2-).

From the Stockholm 2 cohort, fresh-frozen tumour tissues were available for DNA and RNA preparation, from 207 (Study 1) and 93 samples (Study 3), respectively. For Study 2, DNA was available from 29 breast tumours with 11q13 amplification, and 11q13 amplification was defined as four or more gene copies of CCND1 and/or PAK1 and/or RPS6KB2 ([74] and Study 1), whereof 23 was available for RNA extraction and Real-Time PCR. From the Stockholm 3 cohort, 912 paraffinembedded samples were available for TMA preparation (Study 1 and Study 3). As a result of routine mammographic screening, breast tumours are at present detected at a very early stage, and today 75 $80 \%$ of diagnosed tumours are node-negative. The Stockholm 3 cohort is therefore representative for the present clinical situation, whereas the Stockholm 2 cohort represent higher stage cancers.

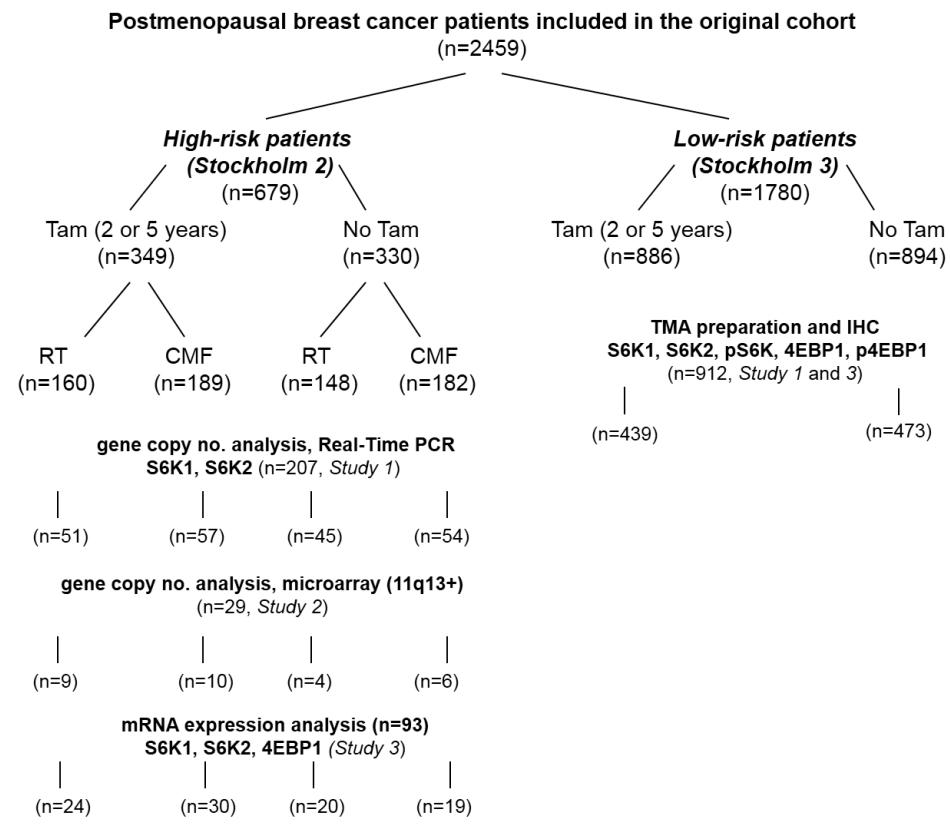

Figure 14 Patient flow through the studies (Tam: tamoxifen 2 or 5 years, RT: radiotherapy, CMF: cyclophosphamide methotrexate 5-fluorouracil chemotherapy, TMA: tissue microarray, IHC: immunohistochemistry). 
Table 4 Patient characteristics of the cohorts used in the studies.

\begin{tabular}{|c|c|c|c|c|c|}
\hline & $\begin{array}{l}\text { Stockholm } 2 \\
(n=207)\end{array}$ & $\begin{array}{l}\text { Stockholm } 3 \\
(n=912)\end{array}$ & $\begin{array}{l}\text { Van de Vijver } \\
(n=295)\end{array}$ & $\begin{array}{l}\text { Uppsala } \\
(n=251)\end{array}$ & $\begin{array}{l}\text { Karolinska } \\
(n=159)\end{array}$ \\
\hline & & & n (\%) & & \\
\hline $\begin{array}{c}\text { Tumour size } \\
<20 \mathrm{~mm} \\
>20 \mathrm{~mm} \\
\text { missing }\end{array}$ & $\begin{array}{l}83(40.1) \\
124(59.9) \\
0(0)\end{array}$ & $\begin{array}{l}697(76.4) \\
189(20.7) \\
26(2.9)\end{array}$ & $\begin{array}{l}155(53.0) \\
140(47.0) \\
0(0)\end{array}$ & $\begin{array}{l}127(50.6) \\
124(49.4) \\
0(0)\end{array}$ & $\begin{array}{l}97(61.0) \\
60(37.7) \\
2(1.3)\end{array}$ \\
\hline $\begin{array}{c}\text { Lymph node } \\
\text { status } \\
- \\
+ \\
\text { missing }\end{array}$ & $\begin{array}{l}22(10.6) \\
185(89.4) \\
0(0)\end{array}$ & $\begin{array}{l}912(100) \\
0(0) \\
0(0)\end{array}$ & $\begin{array}{l}151(51.2) \\
144(48.8) \\
0(0)\end{array}$ & $\begin{array}{l}158(62.9) \\
84(33.5) \\
9(3.6)\end{array}$ & $\begin{array}{l}94(59.1) \\
60(37.7) \\
5(3.1)\end{array}$ \\
\hline $\begin{array}{c}\text { Grade } \\
1 \\
2 \\
3 \\
\text { missing }\end{array}$ & N/A & $\begin{array}{l}142(15.6) \\
438(48.0) \\
169(18.5) \\
163(17.9)\end{array}$ & $\begin{array}{l}75(25.4) \\
101(34.2) \\
119(40.3) \\
0(0)\end{array}$ & $\begin{array}{l}67(26.7) \\
128(51.0) \\
54(21.5) \\
2(0.8)\end{array}$ & $\begin{array}{l}28(17.6) \\
58(36.5) \\
61(38.4) \\
12(7.5)\end{array}$ \\
\hline $\begin{array}{c}\text { ER } \\
- \\
+ \\
\text { missing }\end{array}$ & $\begin{array}{l}61(29.5) \\
144(69.6) \\
2(1.0)\end{array}$ & $\begin{array}{l}195(21.4) \\
689(75.5) \\
28(3.1)\end{array}$ & $\begin{array}{l}69(23.4) \\
226(76.6) \\
0(0)\end{array}$ & $\begin{array}{l}34(13.5) \\
213(84.9) \\
4(1.6)\end{array}$ & $\begin{array}{l}29(18.2) \\
130(81.8) \\
0(0)\end{array}$ \\
\hline $\begin{array}{c}\boldsymbol{P g} \boldsymbol{R} \\
- \\
+ \\
\text { missing }\end{array}$ & N/A & $\begin{array}{l}380(41.7) \\
415(45.5) \\
117(12.8)\end{array}$ & N/A & $\begin{array}{l}61(24.3) \\
190(75.7) \\
0(0)\end{array}$ & N/A \\
\hline $\begin{array}{c}\text { HER2 } \\
- \\
+ \\
\text { missing }\end{array}$ & $\begin{array}{l}146(70.5) \\
56(27.1) \\
5(2.4)\end{array}$ & $\begin{array}{l}739(81.0) \\
93(10.2) \\
80(8.8)\end{array}$ & N/A & N/A & N/A \\
\hline
\end{tabular}




\section{Cell culture and RNA interference as an in vitro model for breast cancer}

In the late 1940s, intense research was ongoing in developing approaches enabling culturing of cancer cells in test tubes, which would revolutionise the studies of cancer origin, growth and drug testing [236]. In 1951, George O Gay at John Hopkins, achieved in establishing the first immortal human cell line [237]. The cell line, now referred to as HeLa, named from the patient Henrietta Lacks, was derived from a cervix adenocarcinoma and is today the most wide-spread human cell line. It has to date been used in a countless number of research studies as an in vitro model of human cancer, but also in studies of other diseases. Since 1962, the American Type Culture Collection (ATCC), a private, non-profit biological resource centre established in 1925, has been the main resource of storing and distributing cell lines to the research community. The use of human cell lines as models of cancer allows studies of mechanisms of drug responses and evaluation of causal relationships at the cellular level. However, the accuracy of in vitro models for cancer in vivo is continuously debated.

The phenomenon of RNA interference was first described by Fire and Mello in 1998, who showed that double-stranded RNA (dsRNA) can silence gene expression in C. Elegance [238]. RNA interference occurs when the enzyme Dicer cleaves dsRNA to short fragments, 21-23 nucleotides, referred to as small interfering RNA (siRNA). Today, siRNA is synthetically produced and are frequently used to study gene functions in vitro. Research is also continuously ongoing in using siRNA as a new therapeutic method in vivo [239].

In Study 4, the human breast cancer cell line ZR751, expressing high levels of both S6K1 and S6K2 (Cancer cell line encyclopaedia [240] and unpublished observations), was used to explore global gene expression alterations after knock-down of S6K1, S6K2 or both S6K1 and S6K2 simultaneously. In addition, several cell lines were used in Study 1-3 as sources of RNA, DNA and protein, for use as calibrator samples in Real-Time PCR, and for evaluation of antibody specificity using western blot before IHC staining. 


\section{Preparation of tumour tissue}

Appropriate collection of tumour tissue is essential for histopathological evaluation in the clinic, but also to allow research on new potential markers and targets. Today, formalin-fixed paraffin embedded (FFPE) tissues are routinely collected for these purposes, which allow histological studies, mainly by IHC, as described below. However, several molecular biological methods, including microarray and Real-Time PCR still require fresh-frozen tissues to preserve high quality DNA and RNA, although these methods are continuously optimised to allow usage of FFPE tissues. According to the routines recommended by the Swedish breast cancer group [6], all tumour tissues to a possible extent, after informed consent from the patients, should be collected and stored, including blood, fine-needle biopsies as well as tissues from metastases. The latter would improve targeted treatment strategies for metastatic disease.

\section{Tissue microarray (TMA) construction}

Analyses of biomarkers in large materials have increased the need for high throughput techniques. The use of TMAs allows studies of up to 1000 samples in a single experiment, on one microscope slide [241, 242]. Briefly, for TMA preparation core needle biopsies from paraffin embedded tissues are reembedded in new paraffin blocks and the blocks are cut in sections (in our case of $4 \mu \mathrm{m}$ ) and mounted on frost-coated slides.

In the present studies, the protein expressions of S6K1, S6K2, S6K1/2 phosphorylated at T389 (pS6K), 4EBP1 and 4EBP1 phosphorylated at Serine 65 (p4EBP1) were evaluated in the Stockholm 3 cohort by IHC staining of TMAs, which is further described below.

\section{Extraction of genomic DNA and total RNA from fresh-frozen tissue}

Careful extraction of DNA and RNA is essential to receive maximum yield, purity and integrity of biomolecules for downstream molecular biological analyses [243]. In general, the main steps include disruption of cells or tissues, inactivation of nucleases, denaturation of nucleoprotein complexes and extraction of nucleotides through several steps of filtering and precipitation. At present, several kits are commercially available for quick nucleotide extraction, requiring a minimum of harmful chemicals. DNA samples are in general stable, and can be stored at room-temperature during use, whereas RNA samples, due to composition of the molecules, are highly sensitive for degradation and, where possible, should be stored in $-70^{\circ} \mathrm{C}$. In Study 1-4, DNA and total RNA were extracted from fresh-frozen tumour tissue estimated to contain $>50 \%$ cancer cells, as well as from cell lines where mentioned.

For DNA, the Puregene ${ }^{\circledR}$ DNA purification kit was used. Briefly, the tissue was digested in proteinase-K solution, and the cell lysate was treated with RNase. Proteins were precipitated using protein precipitation solution included in the kit and pelleted by centrifugation. The supernatant was collected and DNA was precipitated with isopropanol and pelleted by centrifugation. Finally, the DNA was washed with $70 \%$ ethanol, air-dried and dissolved in sterile water. DNA concentration was 
calculated by the Lambert-Beer law, $A b s=\varepsilon \cdot I \cdot c$ [244, 245], where the absorbance at 260nm was measured using a NanoDrop ND-1000 Spectrophotometer. For subsequent analyses, the DNA was long term stored in $-70^{\circ} \mathrm{C}$.

For RNA preparations, fresh-frozen tumour tissue was homogenised with a microdismembrator or a TissueLyser and total RNA was isolated with the mirVana ${ }^{\mathrm{TM}}$ miRNA isolation kit according to instructions provided by the manufacturer. Purified RNA was dissolved in nuclease-free water with addition of RNAsin ribonuclease inhibitor and stored at $-70^{\circ} \mathrm{C}$. RNA integrity numbers (RIN) [246] and concentrations were assessed with an Agilent 2100 Bioanalyzer. Only samples with RIN values $\geq$ 5 were included in the analysis.

\section{Quantitative Real-Time PCR}

One of the most important contributions to present days molecular biology was the invention of the PCR by Kary Mullis in the 1980s [247]. PCR is a highly sensitive method for efficient amplification of small amounts of specific nucleic acid sequences. Quantitative Real-Time PCR uses the PCR technique for quantification of gDNA or complementary DNA (cDNA). It involves a fluorogenic probe to enable detection of the specific PCR-product in real time, as it accumulates during PCR cycles. The probe is labelled with a reporter dye on the 5'end and a quencher dye on the 3'end (Figure 15 a). As with usual PCR, the reaction mixture is transferred between three temperatures during each PCR-cycle. At the first temperature $\approx 95^{\circ} \mathrm{C}$, double-stranded nucleic acids denature and become single stranded. At the annealing temperature $\approx 55^{\circ} \mathrm{C}$, primers and probes attach to the templates. When bound to the target sequence, the probe reporter dye is quenched. DNA-synthesis is initiated when the temperature is set just below the optimum for Taq polymerase, usually $\approx 74^{\circ} \mathrm{C}$ (Figure 15 b). During the extension phase of the PCR, the probe will be degraded by the DNA polymerase 5'nuclease activity. This results in separation of the reporter dye from the quencher dye and a subsequent increase in fluorescence (Figure 15 c). During the PCR cycles, the fluorescence is constantly measured, and the increase is proportional to the quantity of the nucleic acid product in each sample. Thus, the higher the original quantity of nucleic acid target in the sample, the earlier (in PCR-cycles), the reporter signal reaches a detectable level (threshold cycle, Ct-value). The fluorescence signal is usually displayed as an amplification plot (Figure $15 \mathrm{~d}$ ).

For the present studies, reverse transcription of RNA was performed using the high-capacity cDNA reverse transcription kit (Applied Biosystems) with $200 \mathrm{ng}$ total RNA in reactions of $20 \mu 1$ according to manufacturer's instructions. DNA or RNA quantity of each sample was estimated by using the standard curve method (Study 1), or the $\Delta \Delta \mathrm{Ct}$ method (Study 2-4). To adjust for differences in total nucleic acid input, the Ct-values were normalised against the copy number or expression levels of endogenous control genes in each sample. To enable comparison between different runs, a calibrator sample from the same cell line was added on each PCR plate. 
a)

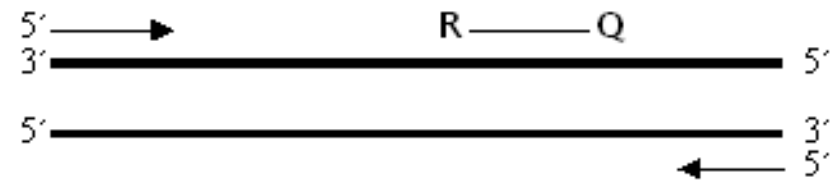

b)

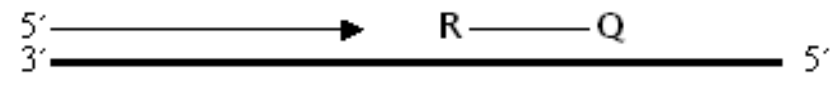

c)

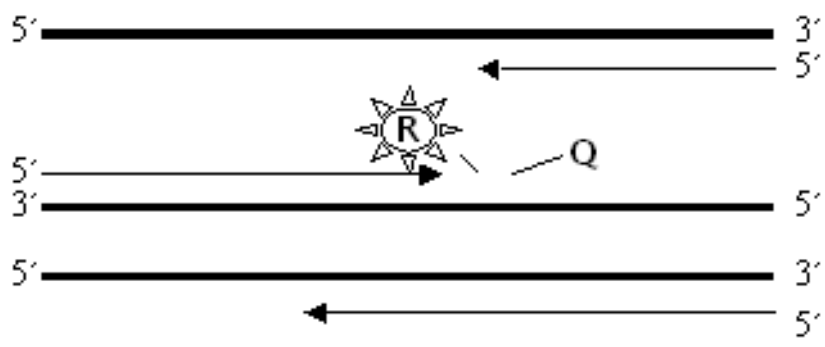

d)

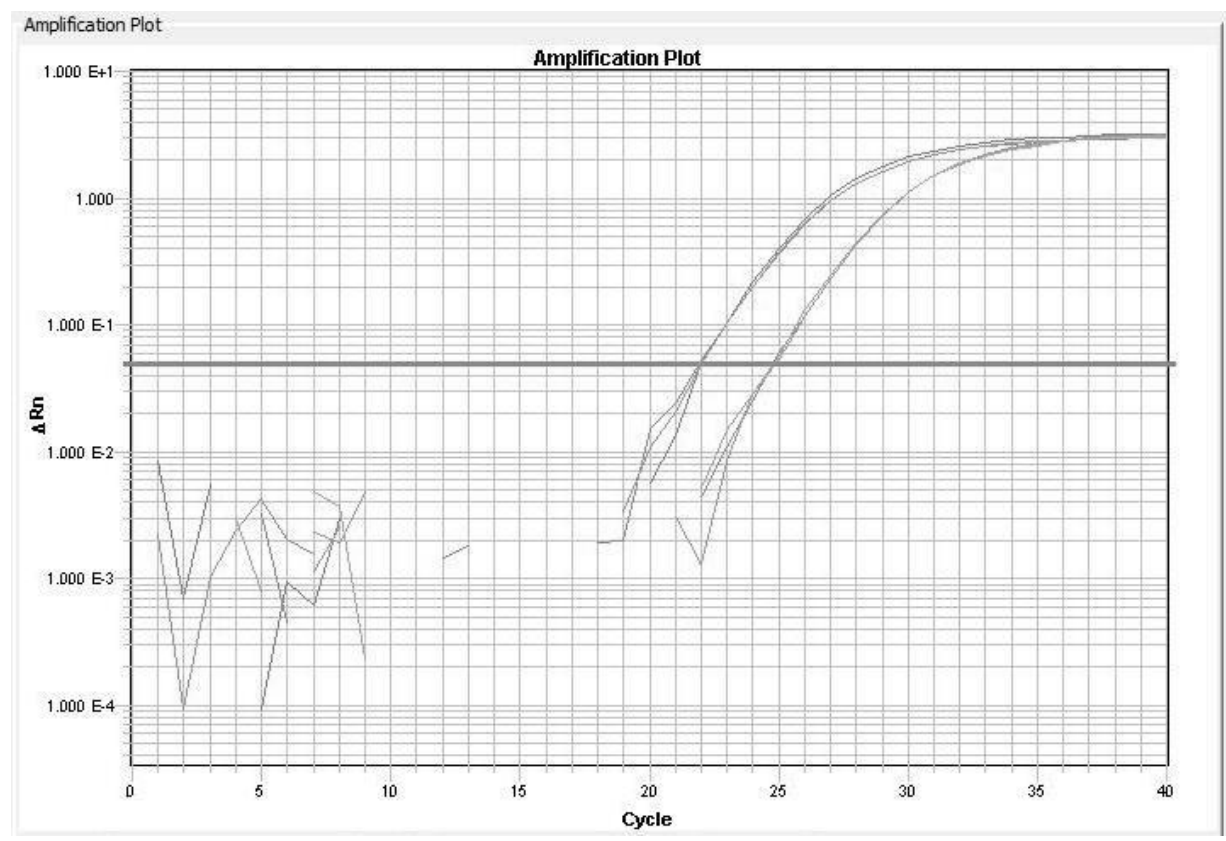

Figure 15 Principles of Real-Time PCR. Primers and probe anneal to the target sequence (a). DNA polymerase extends the primers (b). The polymerase exonuclease activity cleaves the probe and separates the reporter from the quencher, allowing the reporter fluorescence signal to increase (c). Example of a Real-Time PCR amplification plot: $x$-axis $=$ PCR cycle, $y$-axis: fluorescence intensity (logaritmic) (d) (R=reporter dye, $Q=$ quencher dye). 


\section{Immunostaining}

Since the first description by Albert Coons in 1941 of an antibody-based method for specific detection of a protein in tissues or cells [248], the immunostaining methods have developed extensively and are now routinely used for clinical purposes as well as in research. In this project, immunohistochemistry was used to evaluate the expression of S6K2 (Study 1), 4EBP1 and p4EBP1 (Study 3), as well as S6K1 and pS6K [249] in the Stockholm 3 breast cancer material. In addition, western blot was used for validation of antibody specificity in both studies. The following sections shortly describe these methods.

\section{Western blot}

In 1975, Edwin Southern invented a method for detection of specific DNA sequences in samples, subsequently named Southern blot [250]. This was soon followed by similar methods for RNA and protein detection, acronymous named Northern and Western blot [251], respectively.

The western blot method includes separation of molecules according to size using gel electrophoresis, followed by transfer of molecules to a nitrocellulose membrane allowing detection with the use of protein specific antibodies. This method is commonly used for studies of in vitro alterations in protein expression or phosphorylation levels during different circumstances. Size separation allows evaluation of antibody specificity towards an epitope or protein with known size, however the sensitivity is rather low and large differences in expression levels are needed to qualitatively distinguish between protein amounts. Samples are denatured before electrophoresis, thus native epitopes may not always be detected, sometimes obstacle a comparison with immunohistochemistry. However, when possible, western blot is a method recommended for evaluating antibody specificity, which is used in Study 1 and Study 3. Western blot running procedure was described in the respective publications.

\section{Immunohistochemistry (IHC)}

Due to its simplicity, including low time consuming and availability in using FFPE tissues, IHC [252] is routinely used by pathologists for clinical evaluation of protein expression in tissue, e.g. assessment of ER, PgR, HER2 and Ki-67 status. A large advantage is also the possibility to distinguish expression levels between different cell types in the tissues, as well as intracellular localisation of the protein. Though, drawbacks under discussion include problems with reproducibility due to different handling of tissues and sometimes lack of antibody specificity, as well as the subjective judgement and absence of possibility to absolute quantification.

In general, the IHC procedure includes tissue fixation, followed by antigen retrieval, blocking of endogenous peroxidases, and blocking of unspecific binding before adding a primary antibody. Thereafter, a secondary antibody commonly conjugated to horseradish peroxidase (HRP) is used for colour development and detection. Finally counterstaining and glass slide mounting is performed. Evaluation of staining should be done by at least two independent observers to increase objectiveness. 
In the present work, the protein expressions of S6K2 (Study 1), 4EBP1 and p4EBP1 (Study 3), as well as S6K1 and pS6K [249] were evaluated in the Stockholm 3 cohort by IHC stainings of TMAs, as further described in the respective publication. 


\section{Microarray}

In the last two decades a genomic revolution has taken place. Scientists have been able to complete the sequences of a variety of organisms and this culminated in 2001 with the release of the human genome [253]. This has led to the beginning of a new genomic era, where this enormous amount of sequence information can be utilised in a wide variety of research fields to increase the understanding of biological mechanisms. In the light of this, the microarray technology has evolved, and appeared to be a valuable tool.

The microarray technology was invented in the late 1980 s by a team of scientists led by Stephen P.A. Fodor [254]. The principle was taken over by the Affymetrix Company in 1992, and in less than a decade commercialised to what today is called the GeneChip ${ }^{\circledR}$ platform [255]. The use of microarrays has expanded a lot during the last decade, and to date the major approaches are genome wide analysis of gene expression, single nucleotide polymorphism (SNP) genotyping, resequencing (analysis of sequence variation) and DNA methylation profiling. In addition to Affymetrix there are today several companies offering different microarray technologies.

In Study 2, Affymetrix SNP Gene Copy number arrays were used to analyse whole genome amplification profiles of 11q13 amplified breast tumours. Furthermore, in Study 4, Affymetrix expression arrays were used for evaluation of global mRNA expression alterations after in vitro silencing of S6K1 and S6K2.

\section{DNA and mRNA arrays for tumour genome screening}

Expression profiling has recently been shown a clinically useful tool for global molecular evaluation of breast cancers [16]. However, a disadvantage with this strategy is that RNA molecules in general are very unstable, making extractions and standardisations difficult. Furthermore, mRNA expression could be highly variable between tissues and within cells during different times, and finding a standard definition of overexpression is therefore challenging. DNA is more stable than mRNA and since specific copy number aberrations seem to be key drivers of tumourigenesis, direct analysis of copy number alterations on a genome wide scale may have a potential to be an alternative or complementary approach for subtype classification, diagnosis and prognosis of breast tumours [256]. An obstructing factor worth mentioning however, is the recently discovered phenomenon of copynumber polymorphism; that is low-level differences in copy numbers of certain chromosomal fragments between normal individuals $[257,258]$. To date, the extent of this genetic variation remains unknown, but it is a factor worth taken into consideration when studying copy number alterations in tumours. 


\section{GeneChip ${ }^{\circledR}$ array technology}

Microarrays are miniature chips covered by multiple oligonucleotides representing a number of selected SNPs or transcripts. The principles of Affymetrix GeneChip ${ }^{\circledR}$ arrays are reviewed in [255]. For DNA sequence analysis, the most recent generation of GeneChip ${ }^{\circledR}$ microarrays are the SNP arrays. This array was initially designed for genotyping and genetic association studies, but was soon shown to be useful for estimation of DNA copy number variations [259]. For global gene expression analysis, several types of arrays are available, depending on the suitable coverage of transcripts, splicing variants etc.

The arrays are usually composed of a $1.28 * 1.28 \mathrm{~cm}$ solid quartz wafer, where 25 -mer DNA oligonucleotides are successively synthesised by photolithography. Each specific oligonucleotide exists in more than one million copies on a specific location on the array, defined as one feature (Figure 16). In turn, each array includes millions of features. On the 250K DNA arrays used in Study 2, each specific feature exists in a probe pair comprised of a so called perfect match (PM) feature that is complete complementary to its target sequence, and a mismatch (MM) feature, containing a single base substitution in the centre. The MM probes are used as internal controls of non-specific binding and by subtracting the signal intensity of the MM probe from the corresponding PM probe, background signal variations can be reduced. On a SNP array, any given SNP is represented by a set of different features, defined as a probeset. A probeset includes features for both the sense and the antisense strand. Additionally, there are probes with a slight variation in the location of the SNP within the nucleotide sequence. For $\mathrm{CN}$ arrays, there are usually five probe pairs ( $2 * 5$ probes) for each strand of each allele, giving a total number of $2 * 5 * 2 * 2=40$ features representing each SNP.

Microarray running procedures and data analyses are described in the respective publication. 


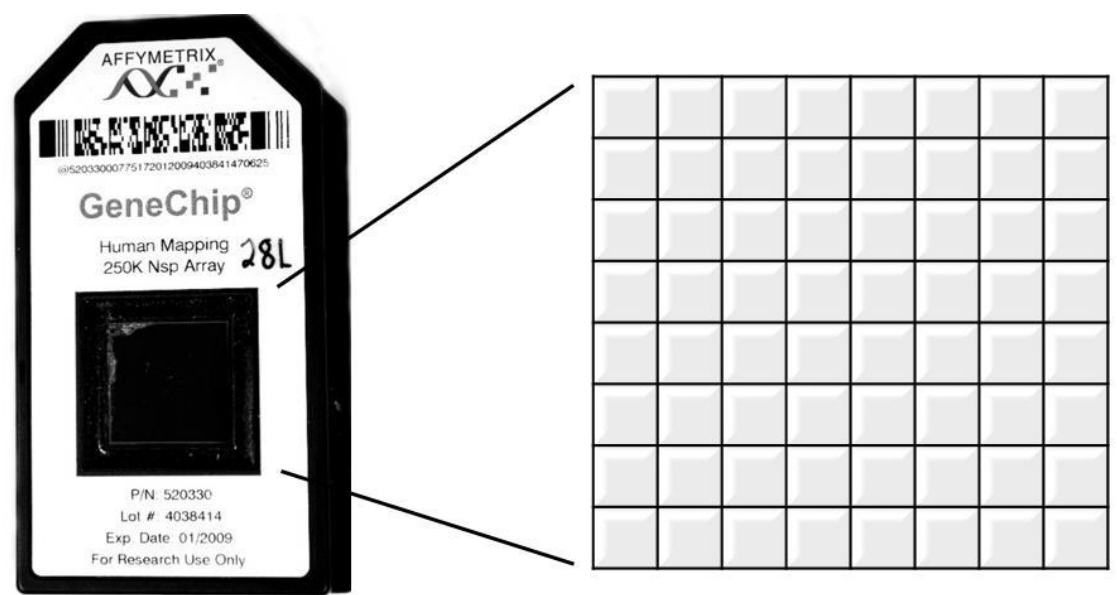

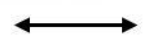

a) GeneChip array $1.28 \times 1.28 \mathrm{~cm}$

b) Millions of features on each array
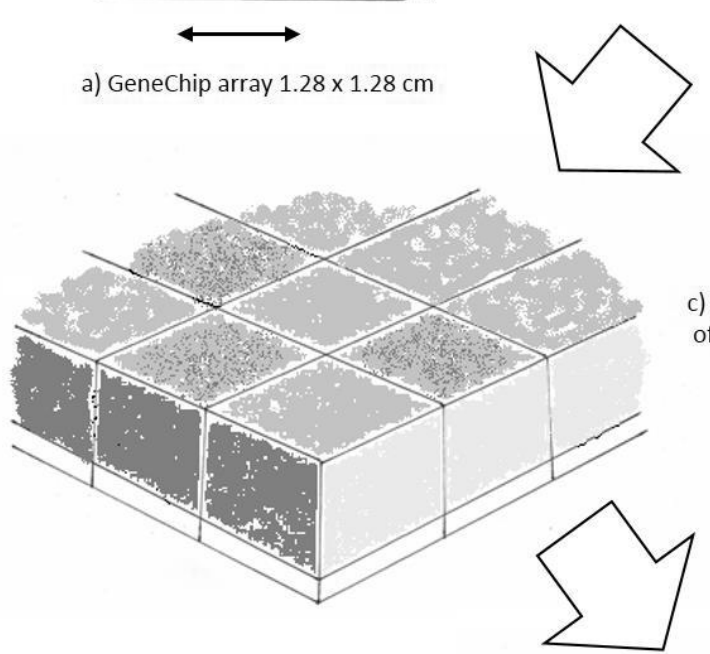

c) Each feature containing millions of identical oligonucleotides

d) 25-mer oligonucleotides
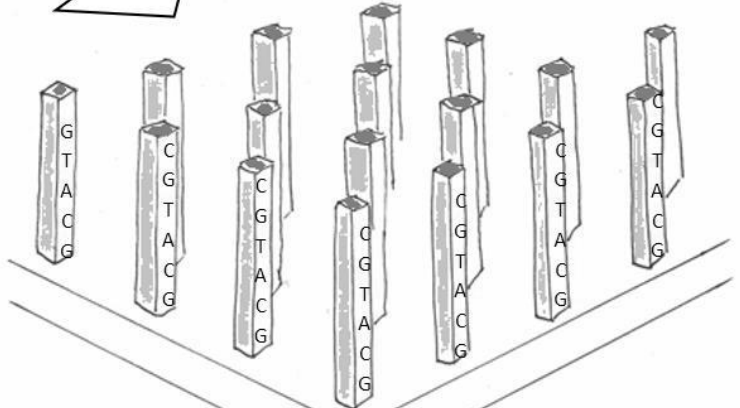

Figure 16 Dissection of a GeneChip array. Each array (a) contains millions of features (b). A feature is a location on the array containing more than one million copies of a specific nucleotide $(c, d)$. Each specific sequence is represented on the chip by several features spread on the array (photo: Tomas Tollwé). 


\section{Bioinformatics databases and analysis tools}

The modern biology with rapidly evolving techniques allowing whole-genome studies, has resulted in a need for large libraries storing and integrating the enormous volumes of produced data, as well as available tools for different types of data analysis. The bioinformatics field is continuously and rapidly expanding. In the following section, an overview of the largest databases will be presented, and a description of tools used in our present analyses.

\section{Genomic and protein databases}

There are today three large main facilities in the US, Europe and Japan for storage and integration of genome sequence information, represented by the National Center for Biotechnology (NCBI) at the National Library of Medicine (NLM), the European Bioinformatics Institute (EMBL-EBI) and DNA database of Japan (DDBJ) [260]. These databases allow all published genomic data to be freely available and they work tightly in cooperation for sharing information between each other. These databases are also linked with corresponding mRNA and protein databases, where NCBI/protein and Uniprot/Swissprot are the largest. For 3D structures of large biological molecules, including proteins and nucleic acids, the Protein Data Bank (PDB) is the only worldwide database of information and coordinates. For development and maintenance of a standardised terminology, the Gene Ontology (GO) bioinformatics initiative was started in 1998 [261]. The GO annotations also classify proteins into its molecular functions, involvement in biological processes and cellular compartments, further promoting data analysis and interpretation. The public web server g:Profiler [262, 263] was used to predict pathways and cellular functions enriched in the array data in Study 4.

\section{Tools for browsing genomic data, sequence alignments and PCR primer design}

Bioinformatics tools for browsing genomic data, as well as sequence alignments of DNA and protein are utilised in many biological fields and several genomic browsers for easy viewing of genomic data have been developed. In Study 2, the NCBI tool MapViewer was applied for whole-genome mapping of known genes within regions of gains and losses based on SNP array data, whereby positions of genes were received from the Human genome build 36, ver3. For sequence alignments, three main algorithms are at present used for this purpose; basic local alignment search tool (BLAST), Fast All (FASTA) and ClustalW [260]. In the present project, the BLAST tool was used in Study 1, whereas both BLAST and and ClustalW were used in Study 4. Primers and probes for S6K1 and S6K2 gene amplification analysis were designed (Study 1) using the software Primer Express version 1.5a (Applied Biosystems) and specificity was controlled by comparison to sequences in the Genebank using BLAST. Protein sequence alignment of S6K1 and S6K2 (Study 4) was performed in order to pinpoint similarities and differences between the primary structures of the proteins. 


\section{Homology modelling of protein 3D structures}

For a protein that is homologous to $>50 \%$ to another protein with known $3 \mathrm{D}$ structure, it is possible to predict its structure with a high degree of confidence using in silico homology modelling [264]. Basically, the method is built on the assumption that native proteins form the most stable conformation in a state where the free-energy is minimised [265, 266]. In addition, tertiary structures are often more conserved than primary structures, thus similarities at the sequence level are often indicative of higher homologous three-dimensional structures. As previously mentioned, in the S6K1 and S6K2 kinase domains approximately $80 \%$ of the residues are conserved. In Study 4, the threedimensional structure of the S6K2 kinase domain was modelled according to standard procedures with the two different S6K1 crystal structures, (PDB ID: 3A62 and 4L3J) as templates, using ICM (Molsoft LLC, San Diego, CA, USA) and Prime (Prime version 3.1, Schrödinger LLC, New York, NY, UA, 2012), respectively. Briefly, the sequences of the templates and S6K2 were initially aligned using Prime and the alignment was then refined using ClustalW. Then a secondary structure prediction was performed and subsequently the three-dimensional model was built using an energybased algorithm. The last step was refinement of loops that lacked electron density in the template (one in this case). The ligands that were co-crystallised with S6K1 were removed prior to homology modelling. The proteins were visualised using PyMol (Schrödinger LLC) and the pairwise root-meansquare-deviation (RMSD) between the template (S6K1) and model (S6K2) was calculated using the SuperPose webserver [267].

\section{Public microarray datasets}

The intervention of microarrays, allowing whole genome studies, resulted in a need for large libraries storing the enormous volumes of produced data. Subsequently, in 2000, NCBI at NLM established the Gene Expression Omnibus (GEO) database [268]. This was followed by the ArrayExpress database, founded by the EMBL-EBI [269, 270]. The use of microarrays soon became routine in medical research, and to standardise the data for publication, the Minimum Information About a Microarray Experiment (MIAME) guidelines were proposed [270]. These guidelines were in 2002 accepted by the Nature journals, proposing that all microarray data should be deposited in either the GEO or ArrayExpress databases were they should be publically available. This allows critical evaluation of published microarray data, as well as free accession for use in other studies. To date, GEO and Array Express are the largest microarray data archives, containing more than 40000 experiments each.

In Study 3 and 4, public available datasets encompassing preprocessed mRNA expression data were used for three cohorts, further referred to as van de Vijver cohort $(\mathrm{n}=295)$ (available at: http://bioinformatics.nki.nl/data.php), Uppsala cohort (n=236) (NCBI/GEO: GSE3494) and Karolinska Institute cohort ( $\mathrm{n}=159)$ (NCBI/GEO: GSE1456) [271-273]. The van de Vijver cohort comprises 295 consecutive patients, primary stage I or stage II breast cancers from the fresh-frozentissue bank of the Netherlands Cancer Institute, and were initially used for evaluation of the 70-gene profile for predicting prognosis [273]. The Uppsala cohort included 236 consecutive patients mainly from the Uppsala County, Sweden, 1987-1989 [271]. The original study aimed to investigate the transcriptional fingerprint of p53 mutated tumours. The 159 samples available in the Karolinska cohort, derived from a larger cohort including all breast cancers operated at the Karolinska Hospital 
1994-1996, where the aim was to identify gene expression profiles of poor prognosis for the addition of adjuvant therapies [272].

\section{Statistics}

For estimation of relationships between different variables in two groups, Fishers exact test [274], or Chi2 tests were used (Study 1). For ranked variables, Spearman's Rank order correlation [275] was applied (Study 1-4). Multiple regression analysis was utilised in cases where one dependent variable was correlated to multiple independent variables (Study 3). The Kaplan-Meier product limit method [276] was used to estimate the cumulative probabilities of local recurrence-free survival (LRFS), distant recurrence-free survival (DRFS) or breast cancer survival (BCS) (Study 1-4). Differences between the curves were evaluated with the Log-rank (Mantel-Cox) test [277] (Study 1-4) or Gehan's test for multiple groups (Study 3). For univariate and multivariate analysis of event rates, as well as interaction analysis, Cox proportional hazard regression was used [278] (Study 1-4).

To give a comprehensive view of the 11q13 amplification patterns across all samples and identify regions of significant aberrations, the principles of a statistical approach referred to as Genomic Identification of Significant Targets in Cancer (GISTIC) was applied [279] (Study 2). GISTIC methodology is based on the assumption that both the frequencies of occurrence, and the amplitude of the aberrations, are corner stones in the development of functionally important genomic changes. In this case, the mean value of the smoothed $\log 2$ ratio of each SNP was calculated across all samples and plotted against genomic location. Resulting peaks of maximal amplitude are indicative of regions harbouring driving oncogenes.

Student's t-test was utilised when comparing differences in gene expression levels in public datasets between different patient groups (Study 4). To evaluate differences in gene expression after in vitro siRNA knock-down of S6K1 and/or S6K2 (Study 4), cell intensity data was processed by the software Genespring (Agilent). Raw data was normalised to the median of the control samples using the Robust Multi-array Average (RMA) summarisation algorithm [280]. Paired t-test was used to estimate significant differences in gene expression after silencing of S6K1 and/or S6K2 in comparison to scrambled siRNA. 


\section{RESULTS AND DISCUSSION}

The prognosis of patients diagnosed with breast cancer has been considerably improved in the latest 25 years, as a result of continuous development of diagnostics and treatment regimens. However, tumour diseases, for woman mainly lung cancer and breast cancer, still constitute the second most common causes of death in developed countries, following heart diseases. A future utopia is to develop more individualised therapy strategies, to further increase breast cancer survival, but also to decrease the risk of severe side-effects of unnecessary treatments. As previously mentioned, humans carrying mutations leading to reduced IGF $1 / \mathrm{mTOR}$ signalling have been shown resistant to development of malignant tumours as well as diabetes, implicating this pathway as one major factor at the cross-road of these diseases [94]. A new treatment regimen constituting a combination of mTOR inhibitor with endocrine therapy was recently clinically approved for advanced breast cancers [7]. Although significant benefit for this combination treatment is evident, counteracting feedback mechanisms are assumed to diminish the effects of the present day's rapaloges. An increased knowledge of the intracellular pathways involved in the mTOR signalling circuit, and its role in different tissues and during different conditions and states, would be potentially helpful in the future prevention and treatment of cancer as well as diabetes. In the present project, we have evaluated downstream effectors of mTOR signalling, mainly S6K1, S6K2 and 4EBP1, with the purpose of finding new potential disease-specific clinical markers and treatment targets for breast cancer.

\section{The mTOR effector $S 6 K 2$ is a new candidate oncogene in the 11 113 amplicon in breast cancer}

When the present project was initiated, previous investigations focusing on S6K1 had showed its corresponding gene $R P S 6 K B 1$, located at the chromosomal region $17 \mathrm{q}$ in the neighbourhood of $E R B B 2$, to be amplified in breast cancer [61, 62, 281]. RPS6KB1 amplification and high protein expression had also been associated with an adverse prognosis [61-63, 281], indicating the mTOR target as a new candidate driver oncogene in the $17 \mathrm{q}$ amplicon. Regarding the homologous protein S6K2, few studies had been conducted, but two reports indicated that the protein levels of S6K2 may be increased in breast tumours [181,282]. The location of the corresponding gene RPS6KB2 in the $11 \mathrm{q} 13$ area, harbouring several known oncogenes amplified in breast cancer, prompted us to investigate the possibility of this gene to be amplified in breast cancer (Study 1). In this project involving 207 stage II breast cancers, RPS6KB2 gain was found in 21.3\% (44/207) of the tumours, whereof amplification defined as at least four gene copies was detected in $4.3 \%(9 / 207)$ of the tumours. Univariate and multivariate analyses indicated a connection between RPS6KB2 amplification and poor outcome in the patient group, independently of other clinicopathological factors and amplification of the neighbouring oncogene CCND1 (Figure 17). In a small cohort of 23 patients, the correlation between $R P S 6 K B 2$ gene copy number and mRNA expression levels was also confirmed. Consequently, RPS6KB2 may be considered a new candidate oncogene in the 11q13 amplicon in breast cancer. In a recent publication, similar results have been shown in gastric cancer, where RPS6KB2 amplification was detected and associated with a poor prognosis [283]. 
Previous results regarding RPS6KB1 in breast cancer were also confirmed in our study, showing $R P S 6 K B 1$ gain in $21.4 \%(44 / 206)$ of the tumours, including amplification in $10.7 \%(22 / 206)$, where the former was also predictive of a poor prognosis. Notably, RPS6KB1 and RPS6KB2 gene amplification were mutually exclusive events, suggesting compensatory tumourigenic roles. The combination variable $R P S 6 K B 1$ or $R P S 6 K B 2$ amplification was inversely correlated to the presence of

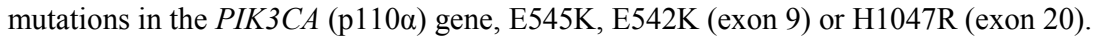



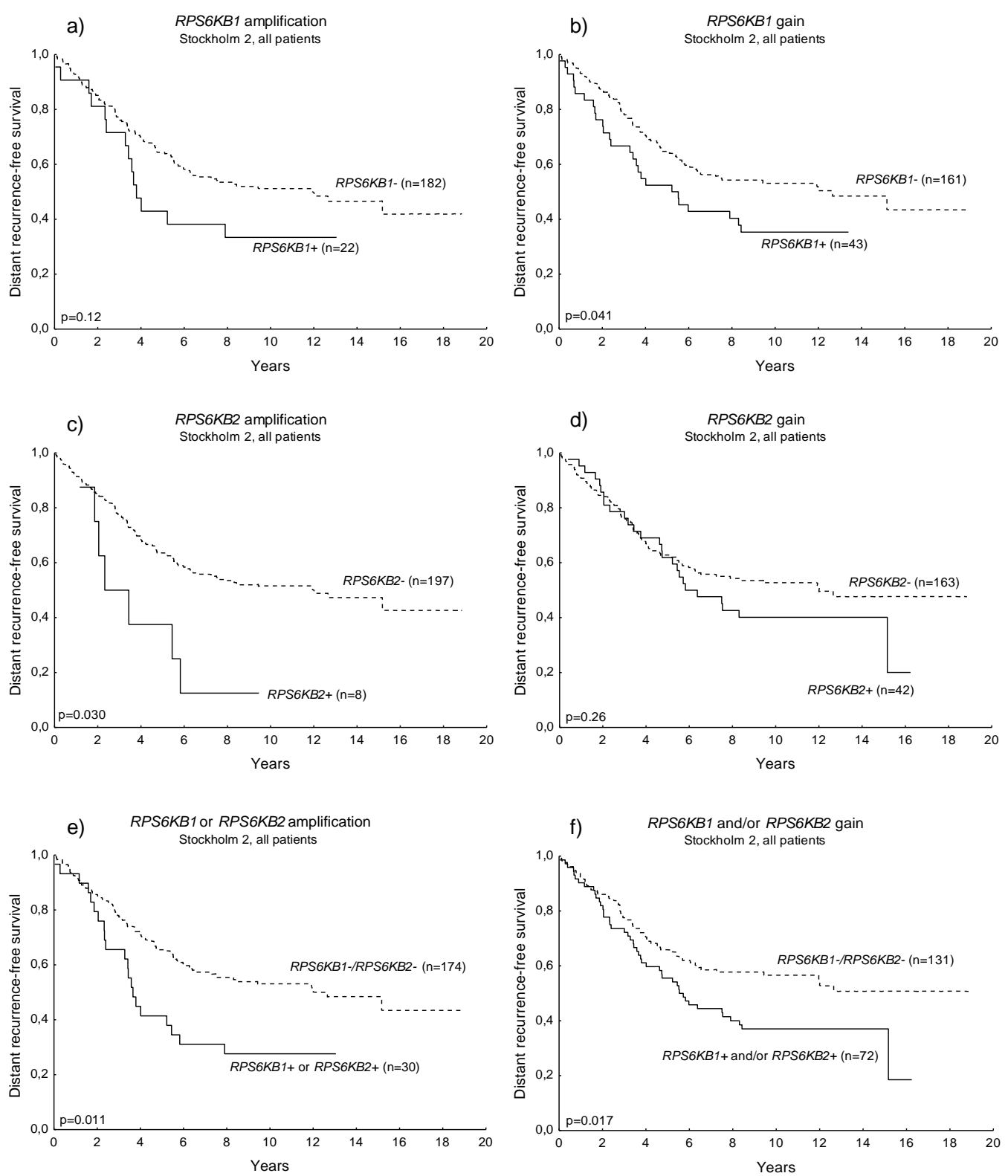

Figure 17 Distant recurrence-free survival among all patients in the Stockholm 2 cohort, in relation to RPS6KB1 amplification (a), RPS6KB1 gain (b), RPS6KB2 amplification (c), RPS6KB2 gain (d), the combination variables RPS6KB1 or RPS6KB2 amplification (e) and RPS6KB1 and/or RPS6KB2 gain (f). (amplification $\geq 4$ gene copies, gain $\geq 3$ gene copies) 
RTK/PI3K signalling is tightly regulated by several feedback mechanisms among others acting on IRS1 [284]. Downstream AKT activation induces a positive loop, enhancing IRS signalling through phosphorylations at certain Serine residues. On the contrary, activated mTOR and S6K1, as well as IKK and JNK diminishes further IRS signalling through phosphorylations at other sites, by reducing RTK and PI3K binding and increasing IRS1 degradation. A new study has investigated the consequences of mutations at different sites of PIK3CA, showing that the E545 mutation in exon 9 leads to downstream signalling through IRS1 independent of upstream receptor activation [285]. When the RPS6KB1 and RPS6KB2 gene amplifications were correlated to the E545K and H1047R mutations, separately, RPS6KB1 was inversely correlated to E545K only (Table 5). This was not evident for RPS6KB2. S6K1, but not $\mathrm{S} 6 \mathrm{~K} 2$, has been shown involved in the negative feedback described above, inhibiting AKT-signalling by phosphorylating IRS1 [222], supporting this finding and indicating separate cellular functions for S6K1 and S6K2.

Possibly partly due to its chromosomal locations, RPS6KB1 gene amplification was associated with HER2-amplification and protein expression, whereas RPS6KB2 was rather connected to ER-positive breast cancer. PI3K/AKT/mTOR signalling has been shown to interfere with ER pathways, affecting the response to ER-targeted therapies in breast cancer [286]. Consequently, the predictive value of RPS6KB1 and RPS6KB2 gene amplification for the outcome of tamoxifen treatment was investigated in the cohort. Benefit from tamoxifen was seen in the group of patients with tumours harbouring $R P S 6 K B 2$ gene gain, whereas no significant response was seen in the RPS6KB2 negative group (Figure 18). For RPS6KB1 amplification or gain, no connection to benefit from endocrine treatment was evident (Figure 18), further supporting a clinical relevant difference between S6K1 and S6K2.

Table 5 Correlations between S6K1 and S6K2 amplification, and mutations in PIK3CA exon 9 or exon 20.

\begin{tabular}{|c|c|c|c|c|c|c|}
\hline \multirow{2}{*}{$\begin{array}{c}\text { PIK3CA } \\
\text { mutation }\end{array}$} & \multicolumn{2}{|c|}{ RPS6KB1 gain n (\%) } & \multicolumn{4}{|c|}{ RPS6KB2 gain n (\%) } \\
\hline & 0 & 1 & & 0 & 1 & \\
\hline Exon 9 & & & & & & \\
\hline 0 & $137(86.2)$ & $44(100.0)$ & & $142(88.2)$ & $40(93.0)$ & \\
\hline 1 & $22(13.8)$ & $0(0)$ & $\mathrm{p}=0.0090$ & $19(11.8)$ & $3(7.0)$ & $\mathrm{P}=0.36$ \\
\hline Exon 20 & & & & & & \\
\hline 0 & $137(86.2)$ & $38(86.4)$ & & $139(86.3)$ & $37(86.1)$ & \\
\hline 1 & $22(13.8)$ & $6(13.6)$ & $\mathrm{p}=0.97$ & $22(13.7)$ & $6(14.0)$ & $\mathrm{P}=0.96$ \\
\hline
\end{tabular}




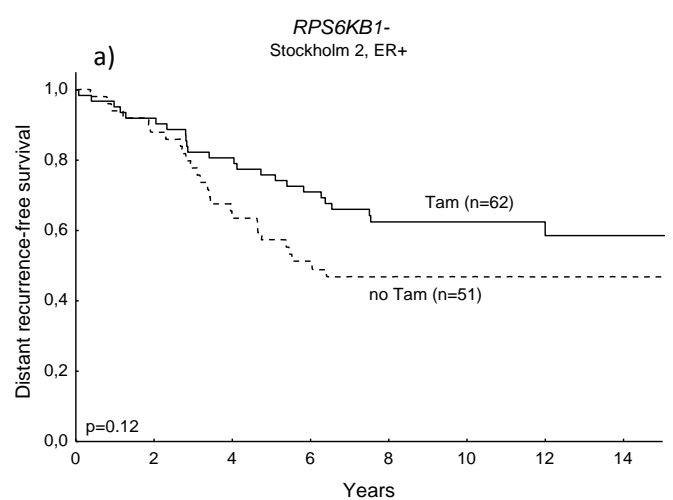

c)

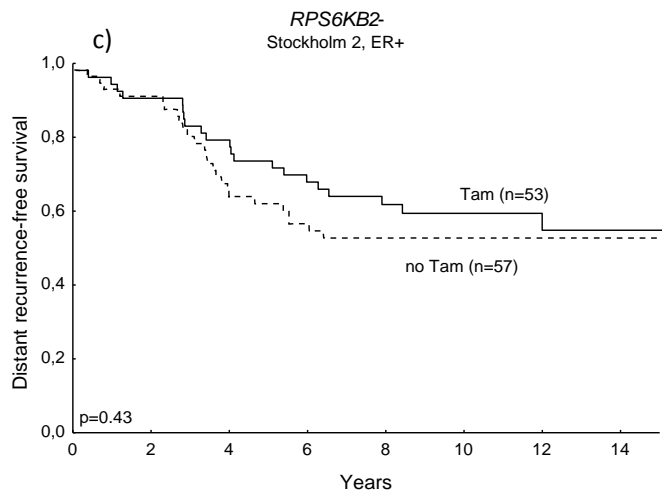

RPS6KB1+

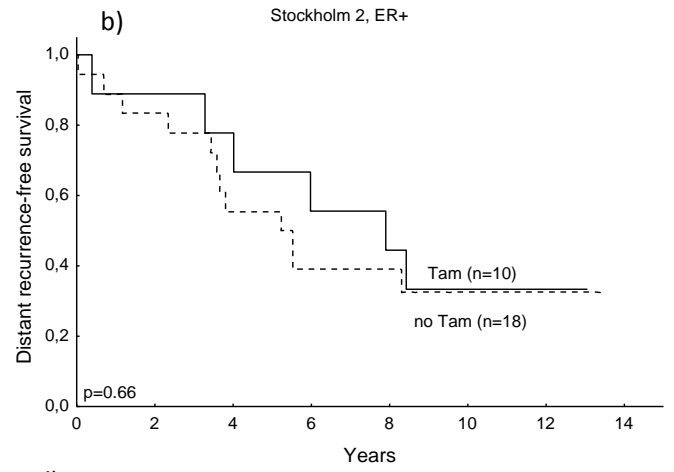

d)

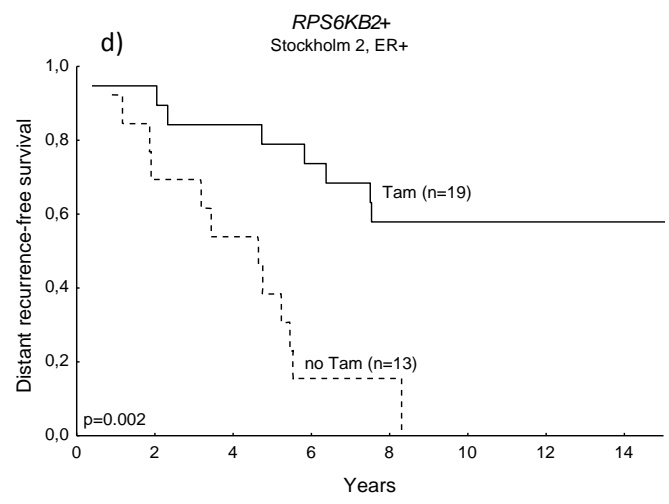

Figure 18 Distant recurrence-free survival among patients treated (Tam) or not treated (no Tam) with tamoxifen, in the groups of patients with ER-positive tumours harbouring normal RPS6KB1 gene copy number (a), RPS6KB1 gain (b), normal RPS6KB2 gene copy number (c) or RPS6KB2 gain (d). (Tests for interaction: RPS6KB1gain vs. tam: $p=0.63$, RPS6KB2 vs. tam: $p=0.065$ ). 


\section{S6K2/4EBP1 coamplification and $m R N A$ coexpression is associated with a poor outcome, indicating a synergy between mTOR targets}

Gene amplification of the 11q13 chromosomal region has been shown clinically relevant in breast cancer, connected to ER positive status, poor prognosis and diverse response to endocrine treatment $[58,72-74,76,78,87]$. Numerous studies of 11q13-amplified tumours have revealed a complex and heterogeneous amplification pattern, likely as a result of several driving oncogenes. In a following project, we aimed to further investigate the clinical relevance of RPS6KB2 amplification in the context of the 11q13 amplicon, as well as its relations to genome-wide copy number alterations (Study 2). Whole-genome SNP arrays were used in a screen of 29 breast tumours with 11q13 amplification, representing a cohort of approximately 200 patients. Assuming that the frequencies of occurrence as well as the amplitude of the aberrations are essential, the statistical method GISTIC [279] allowed estimation of important amplicon cores in the region. Resulting data showed three main amplicon cores in the 11q13 area of these tumours (Figure 19 a). Depending on methodology, selection of tumour samples or cell lines, several different cores in the $11 \mathrm{q} 13$ have been suggested [76, 85, 87, 287-290], but a recent review summing up previous high-resolution studies of the amplicon, confirms our present findings of these three cores, but also an additional one between core 2 and 3 [72]. The selection on tumours harbouring amplification of RPS6KB2, CCND1 and/or PAK1 may explain the absence of the fourth core in our study.

Previous connections between positive ER status and 11q13 amplification were suggested to be restricted to the core including among others CCND1, FGF3, FGF4 and FGF19 in Study 2, using multivariate analysis. The indicated role of cyclin D1 as a cofactor of the ER [291], and CCND1 as an ER-targeted gene [292] support these findings. This also confirmed the previous observation of 11qter loss, including among others the PgR gene along with 11q13 amplification, likely resulting from the breakage-fusion-bridge cycles suggested to form the amplicon [85].

Intriguingly, amplifications at 11q13 are commonly cooccurring with amplifications at $8 \mathrm{p} 12$, and the $11 \mathrm{q} 13 / 8$ p12 coamplification has been associated with a worse outcome than either amplicon alone, suggesting a tumourigenic synergy [59, 77]. In line with this, 8 p12 gain or amplifications were detected in 55.2\% (16/29) of the tumours in the present study (Figure 19 b). Interestingly, when restricting the GISTIC analysis of the $11 \mathrm{q} 13$ amplicon to tumours with or without $8 \mathrm{p} 12$ gain/amplification, the most proximal amplicon, containing among other genes RPS6KB2, mainly appeared in the $8 \mathrm{p} 12$ positive tumours (Figure 20). Multivariate analysis confirmed the $11 \mathrm{q} 13 / 8 \mathrm{p} 12$ coamplification to mainly include the proximal $11 \mathrm{q} 13$ core. 

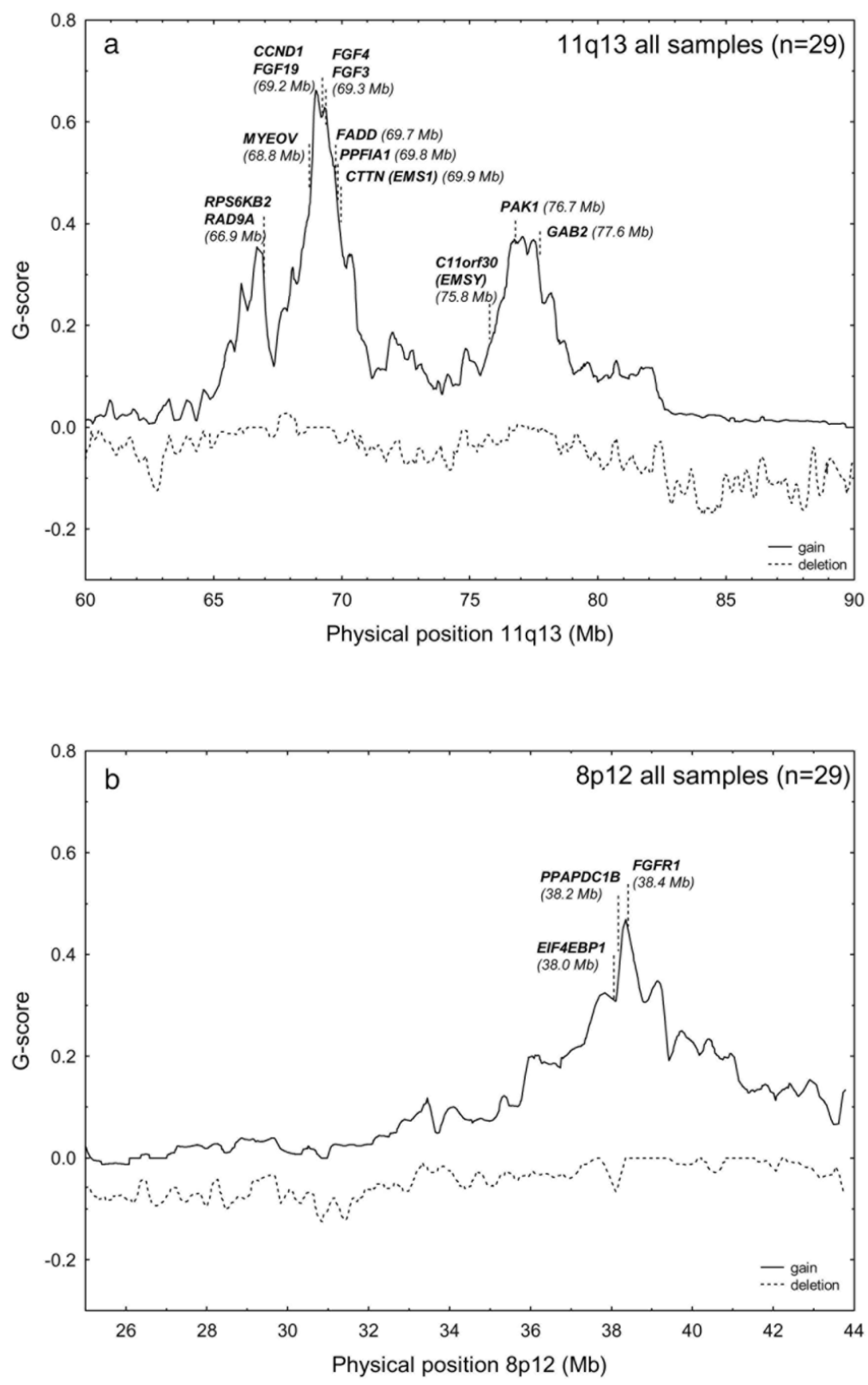

Figure 19 Comprehensive view of the 11q13 amplicon (a) and the 8p12 amplicon (b) in 29 breast cancers and identification of the most significant amplifications, using the principles of GISTIC methodology. 

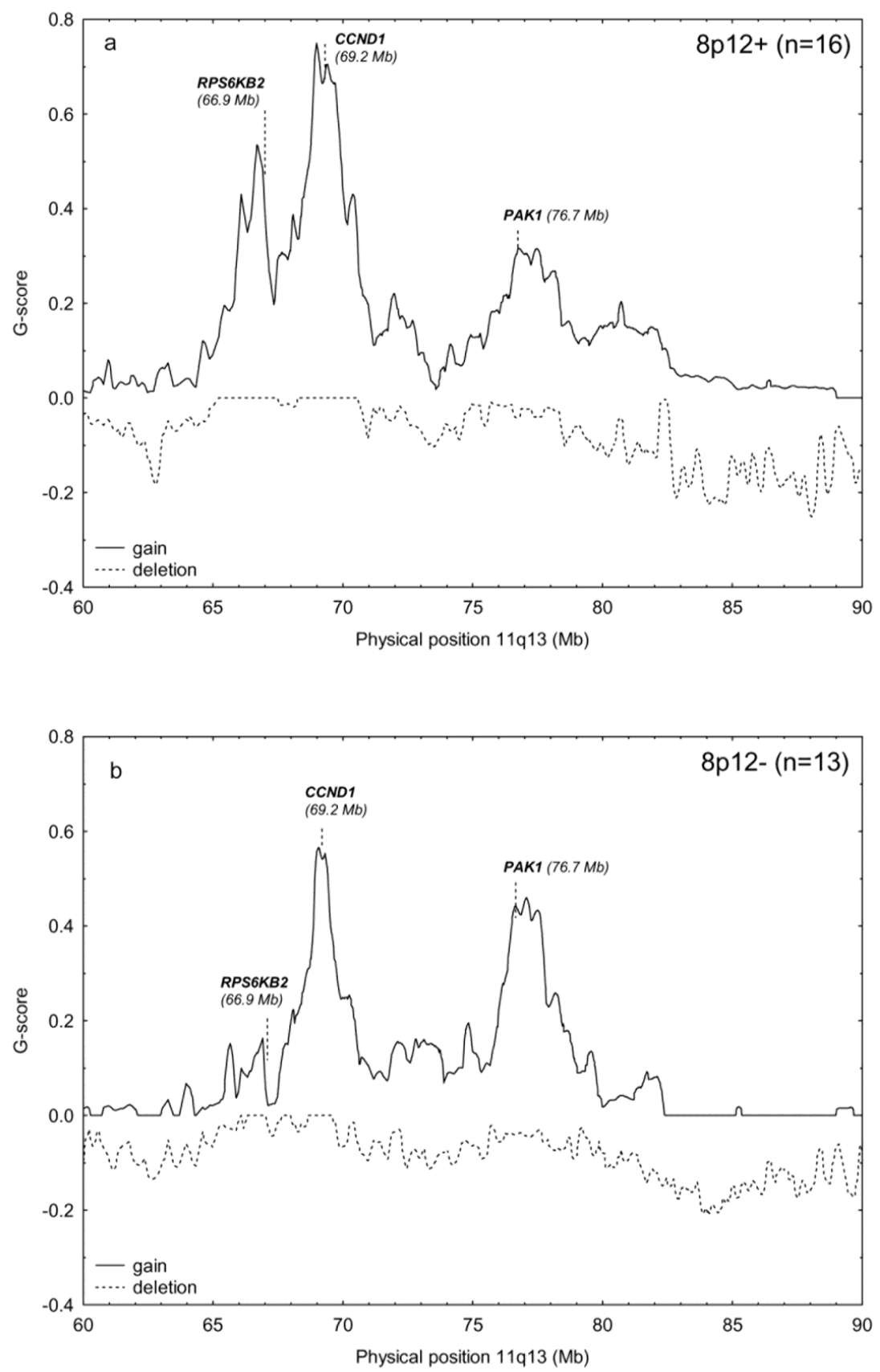

Figure 20 GISTIC view of the 11q13 amplicon in the 29 tumours divided into two groups based on 8p12 status: $8 p 12+\geq 3$ DNA copies (a), 8p12- $<3$ DNA copies (b). 
Previous suggested drivers of the 8p12 amplicon formation are mainly the FGFRI and PPAPDC1B genes $[184,293]$. Due to the location of the mTOR target RPS6KB2 in the $11 \mathrm{q} 13$ core coamplified with $8 \mathrm{p} 12$, one may also deem the EIF4EBP1 gene at $8 \mathrm{p} 12$, encoding the mTOR effector 4EBP1, as interesting. Consequently, 4EBP1 mRNA expression was evaluated along with FGFR1 and PPAPDC1B in the cohort of 11q13 amplified tumours. Correlations between gene copy number and mRNA levels were confirmed for these three genes, as also reported previously [184, 185, 187]. In addition, these were also significantly correlated to S6K2 mRNA. Interestingly, FGFR1 mRNA was also correlated to FGF3, FGF4 and FGF19 mRNA. 8p12 gene amplification, as well as high FGFR1 or 4EBP1 mRNA expression, but not PPAPDC1B was predictive of a poor prognosis in the cohort of patients with 11q13 positive tumours (Figure 21). As previously discussed [72], this confirmed several genes in the 11q13/8p12 amplicons as potential drivers, but also adds the mTOR targets S6K2 and 4EBP1 to this list.
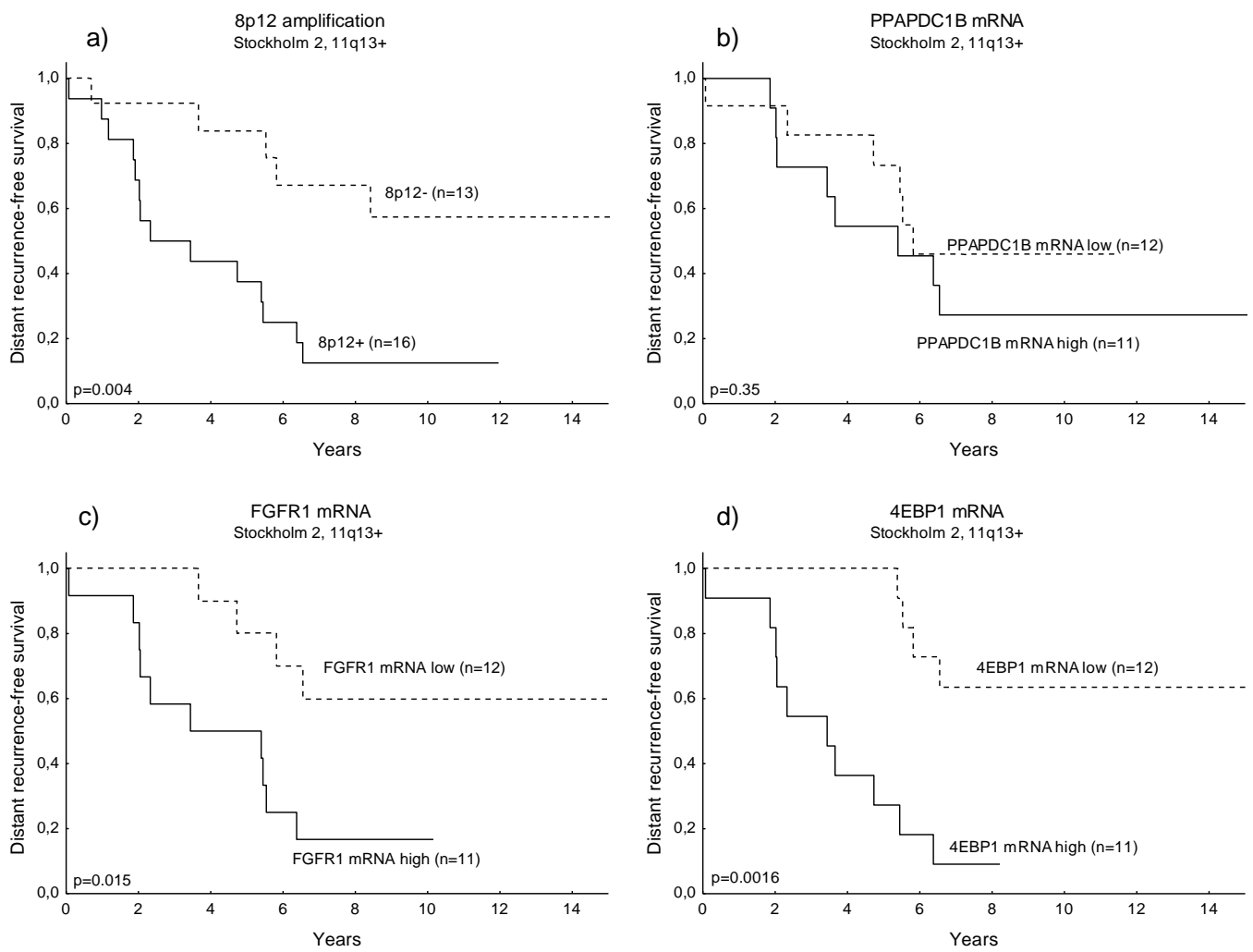

Figure 21 Distant recurrence-free survival among patients with 11 19 positive tumours in relation to; $8 p 12$ gain/amplification (8p12+: $\geq 3$ DNA copies, 8p12-: < 3 DNA copies) (a), high mRNA expression of PPAPDC1B (b), FGFR1 (c) and $4 E B P 1$ (d). The limit for high $\mathrm{mRNA}$ levels was defined by the median for each gene. 
To further evaluate the clinical implications of the S6K2/4EBP1 coexpression, as well as possible divergence between S6K1 and S6K2 in this sense, the mRNA expression levels of S6K1, S6K2 and 4EBP1 were assessed in a larger cohort from the Stockholm 2 material, consisting of 93 available patient samples (Study 3). Here, the strong correlation between S6K2 and 4EBP1 mRNA levels was confirmed, whereas this could not be seen for S6K1 and 4EBP1 (Figure 22).

High mRNA levels of S6K2 and/or 4EBP1 were also associated with an adverse outcome in the patient group (Figure 23). As a validation, the same analyses were performed in three publically available datasets, were the correlations as well as the prognostic value of S6K2/4EBP1 could be confirmed (Figure 22 and Figure 23). For S6K1 mRNA, no prognostic value was evident.

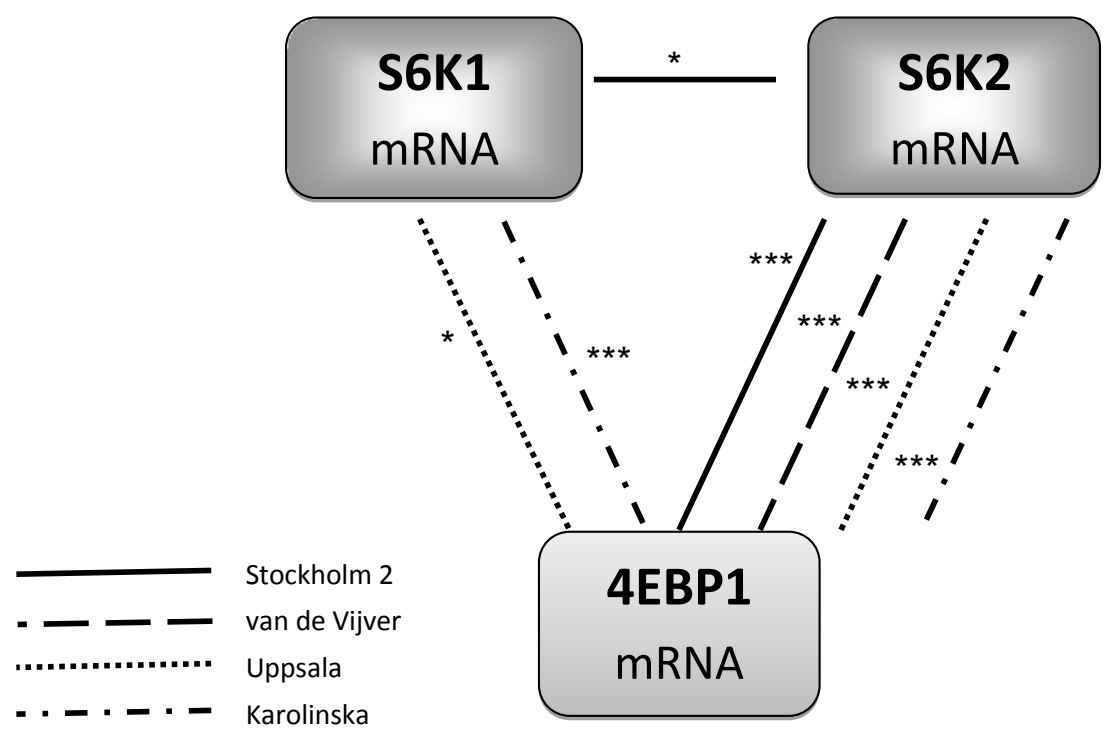

Figure 22 Spearman's rank order correlation evaluating associations between S6K1, S6K2 and $4 E B P 1 \mathrm{mRNA}$ expression (continuous values) in four breast cancer cohorts $\left({ }^{*} p<0.05,{ }^{* *} p<0.01\right.$, $* * * p<0.001)$.

Consequently, the results of these studies suggest an essential distinction between S6K1 and S6K2 as clinical markers, which may result from partly functional differences between these kinases. The role of 4EBP1 in breast cancer may seem contradictive. Phosphorylation of 4EBP1 by mTORC1 promotes dissociation of 4EBP1 from eIF4E, enabling eIF4E to induce protein translation, consequently, 4EBP1 has been considered as a tumour suppressor. However, phosphorylated 4EBP1 has been generally accepted as a marker of activated mTOR signalling and high levels in tumours have been associated with a worse outcome in several malignancies [189, 190, 294, 295]. Results from our studies regarding EIF4EBP1 gene amplification and high mRNA levels as associated with a poor prognosis, indicate a dual role for 4EBP1. Supporting this finding, 4EBP1 has been shown able to bind and stabilise mTORC1, promoting activation of the signalling pathway [192]. 

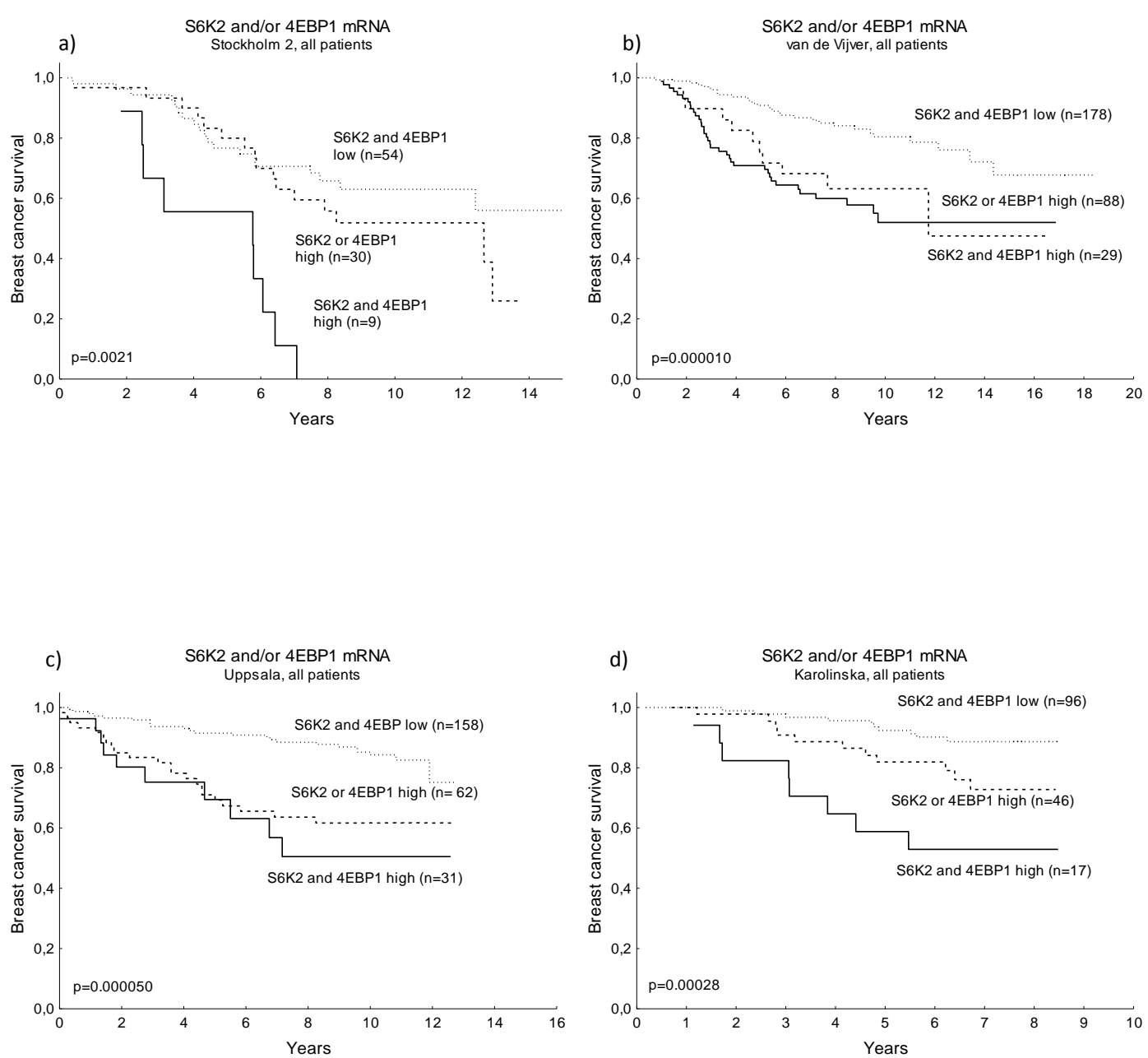

Figure 23 Breast cancer survival (BCS) in relation to high $S 6 K 2$ and/or $4 E B P 1$ mRNA in four cohorts; Stockholm 2 (a); van de Vijver (b); Uppsala (c); and Karolinska (d). 


\section{Whole-genome $m R N A$ profiles of $S 6 K 2$ and $4 E B P 1$ positive tumours are highly overlapping, revealing associations with cell cycle regulators and IGF signalling}

To further evaluate the implicated differences between S6K1 and S6K2 at the transcriptional level, as well as the association between S6K2 and 4EBP1, whole genome-transcriptional profiles of breast tumours expressing high levels of these genes, respectively were investigated (Study 4). The whole genome-profiles of S6K2 and 4EBP1 high tumours showed significant overlaps, whereas few similarities were seen between these profiles and the profiles for S6K1 high tumours. Correlations, positive or negative, of expression levels for certain genes may have several reasons [296, 297]. A causal relationship may be present where one of the factors regulates the expression levels of the other. Coamplification, perhaps as a result of chromosomal colocalisation may be present as well as coregulation by the same transcription factors. In malignancies, coexpression of genes involved in the same biological processes may be a result of an evolutionary tumour promoting advantage, whereas an inverse relationship could result from mutually exclusive roles of the factors. Overlapping expression profiles of S6K2 and 4EBP1, included several factors involved in cell cycle promotion and proliferation, among these CCNB1, CCNB2, CENPA, CDC20 and CDC25B. Notably, the transcription factor E2F1 and several of its targets were strongly associated with S6K2/4EBP1, but not with S6K1, and this could be confirmed in two additional data sets (Figure 24).

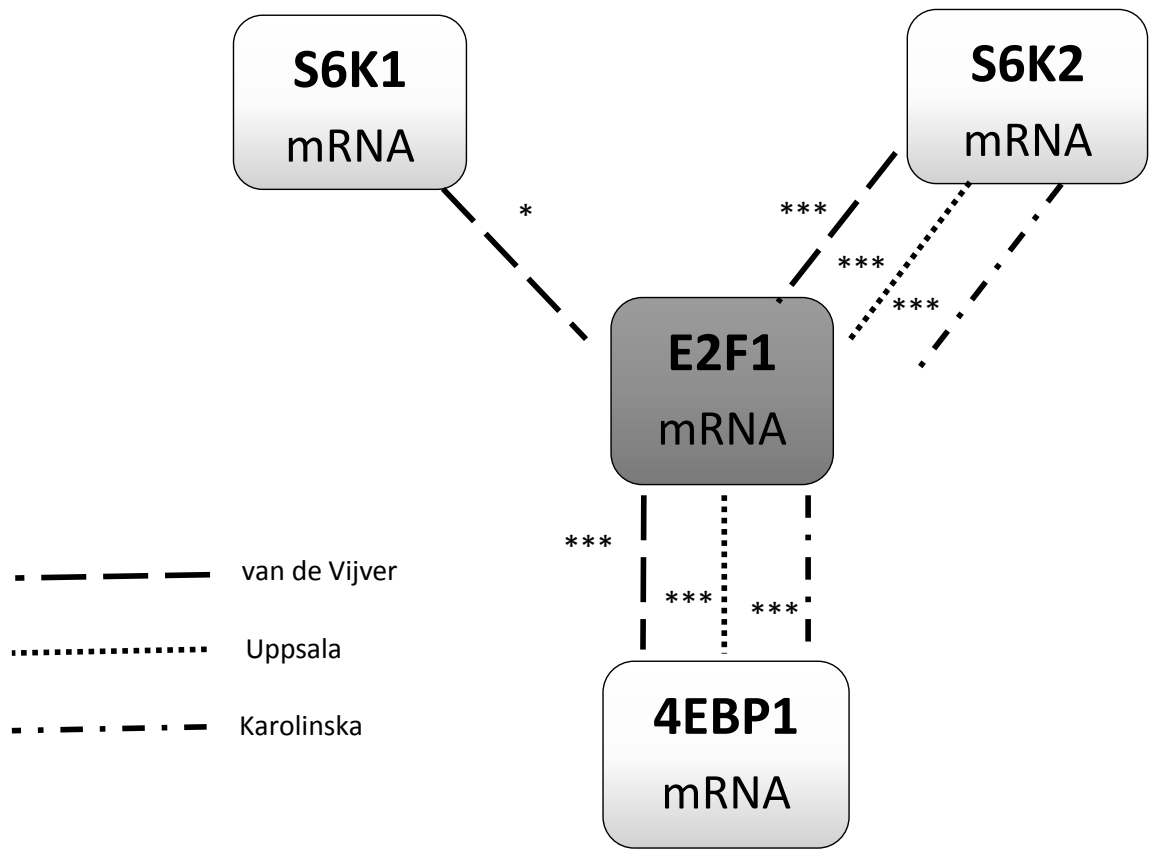

Figure 24 Spearman's rank order correlation evaluating associations between S6K1, S6K2, 4EBP1 and E2F1 mRNA expression (continuous values) in three breast cancer cohorts $\left({ }^{*} p<0.05\right.$, ${ }^{* *} p<0.01$, $* * * p<0.001)$. 
Loss of function of the tumour suppressor $\mathrm{pRb}$, leads to aberrant activity of E2F1, promoting cellular proliferation. The $\mathrm{pRb}$ was the first identified tumour suppressor and its function is lost in many cancers, including $20-35 \%$ of breast cancers [298]. pRb associates directly with the transcription factor E2F1, regulating its activity, through $\mathrm{pRb}$ phosphorylation by cyclin-dependent kinases (CDKs), mainly associated to cyclin D and cyclin E (Figure 5). However, the role of E2F1 in cell cycle regulation and tumourigenesis has long been under debate [299-301] and E2F1 has also been shown a trigger of apoptosis in response to DNA damage. High E2F1 mRNA levels have been associated with a poor outcome in breast cancer [302]. E2F1 activity is regulated by upstream growth factor signalling pathways, and Ras/MAPK as well as PI3K/AKT pathways have been shown to inhibit E2F1 proapoptotic gene transcription [301]. In addition, AKT has been shown a transcriptional target of E2F1, suggesting a positive feedback inhibiting apoptosis. A recent study has linked E2F1 to mTOR signalling, showing that E2F1 can activate mTORC1 and downstream signalling probably by recruiting $\mathrm{mTORC} 1$ to endosomes, independent of AKT and insulin signalling [303].

One of the main genes inversely correlated to both S6K2 and 4EBP1 was IGF1. The inverse relationship with several factors in the IGF signalling pathway could be confirmed in three datasets (Table 6). The IGF/IGFR axis is a main activator of AKT/mTOR signalling [304], indicating that the inverse correlation may reflect a compensatory relationship and mutually exclusive IGF1 or S6K2/4EBP1 levels. IGF1 and IGF2 can signal in an endocrine, paracrine or autocrine manner [304]. IGF1 and IGF2 bind and activate IGF1R, leading to homodimerisation as well as heterodimerisation with the INSR, promoting IRS1 recruiting and downstream PI3K/AKT/mTOR activation. IGF2R, in turn, is considered an inhibitor or IGF signalling by binding IGF2, promoting its degradation. As mentioned in the introduction, absence of systemic IGF signalling seems to protect from cancer as well as diabetes, highlighting this pathway as an interesting therapeutic target. However, side-effects on the normal physiology have so far impeded the use of IGFR inhibitors in the clinic.

The cellular metabolic control performed by the IGF system in normal cells, is directly linked to mTOR activity. Upregulation of intracellular pathways may render the tumour cells insensitive to systemic proliferative and metabolic control through IGF, supporting the inverse correlation between S6K2/4EBP1 and IGF levels. However, in the present study, the earlier implicated prognostic value of S6K2/4EBP1 was shown restricted to tumours expressing high levels of IGFRs or HER2, indicating an important role in growth factor driven tumours (Figure 25). The prognostic value was also confirmed in a multivariate analysis, including several clinicopathological factors as well as E2F1. 
Table 6 Spearman's rank order correlation evaluating associations between S6K1, S6K2, 4EBP1 and $m R N A$ expression of factors in the IGFR signalling pathway (continuous values) in three breast cancer cohorts ( $R$-values for $p<0.01$ highlighted, positive correlations in grey).

\begin{tabular}{|c|c|c|c|c|c|c|c|c|c|}
\hline & & IRS1 & IRS2 & IRS4 & IGF1 & IGF2 & IGF1R & IGF2R & INSR \\
\hline \multirow[t]{3}{*}{ S6K1 } & van de Vijver & -0.09 & -0.05 & 0.09 & 0.02 & -0.23 & 0.02 & -0.08 & 0.03 \\
\hline & Karolinska & 0.12 & -0.11 & -0.21 & -0.13 & -0.18 & 0.05 & 0.08 & 0.01 \\
\hline & Uppsala & -0.05 & -0.10 & -0.10 & -0.13 & -0.14 & 0.03 & 0.25 & -0.04 \\
\hline \multirow[t]{3}{*}{ S6K2 } & van de Vijver & -0.20 & 0.22 & 0.10 & -0.43 & -0.28 & -0.06 & 0.02 & 0.14 \\
\hline & Karolinska & -0.12 & -0.16 & 0.13 & -0.30 & -0.29 & -0.23 & 0.18 & -0.04 \\
\hline & Uppsala & -0.31 & -0.19 & 0.03 & -0.27 & -0.24 & -0.22 & -0.06 & -0.20 \\
\hline \multirow[t]{3}{*}{ 4EBP1 } & van de Vijver & -0.32 & 0.08 & 0.14 & -0.36 & -0.27 & -0.18 & 0.08 & 0.03 \\
\hline & Karolinska & -0.25 & -0.36 & 0.00 & -0.49 & -0.46 & -0.30 & 0.24 & -0.11 \\
\hline & Uppsala & -0.19 & -0.14 & -0.03 & -0.48 & -0.43 & -0.26 & -0.06 & -0.14 \\
\hline
\end{tabular}


a) S6K2 and/or 4EBP1 mRNA IGF1R+ and/or IGF2R+ and/or HER2+

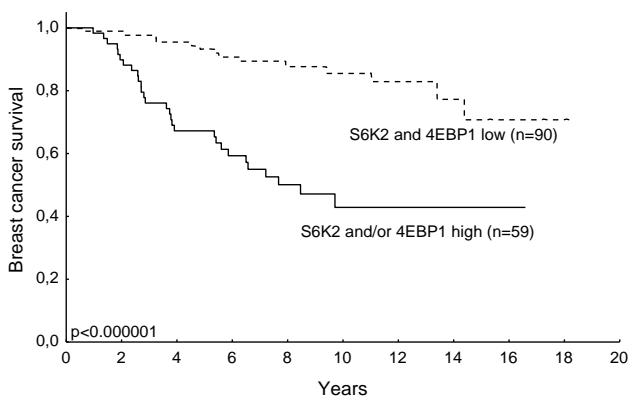

c)

S6K2 and/or 4EBP1 mRNA Karolinska

IGF1R+ and/or IGF2R+ and/or HER2+

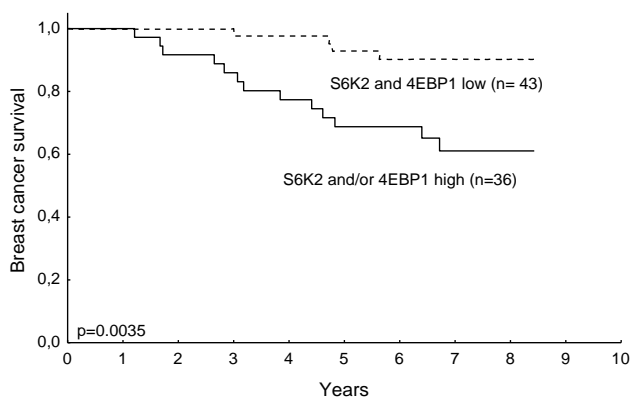

e)

S6K2 and/or 4EBP1 mRNA

Uppsala
IGF1R+ and/or IGF2R+ and/or HER2+

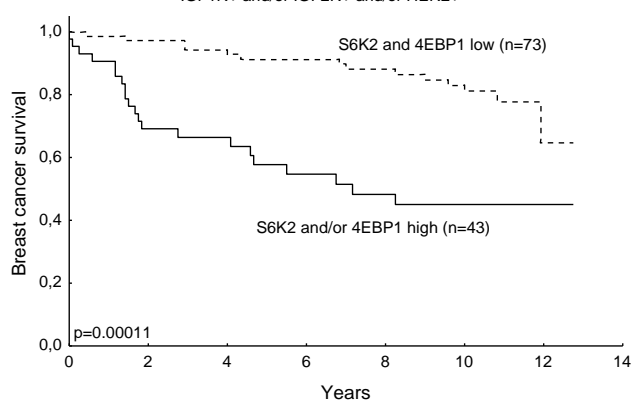

b) S6K2 and/or 4EBP1 mRNA

IGF1R- and IGF2R- and HER2-

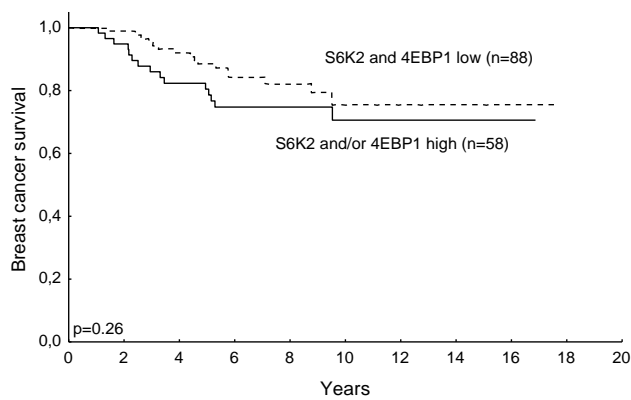

d) S6K2 and/or 4EBP1 mRNA

IGF1R- and IGF2R- and HER2

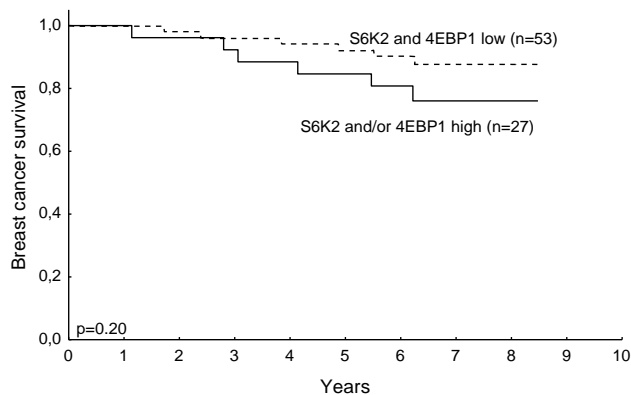

S6K2 and/or 4EBP1 mRNA

Uppsala

IGF1R- and IGF2R- and HER2

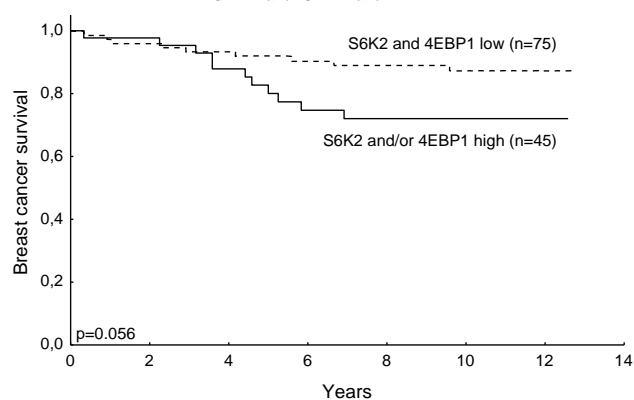

Figure 25 Breast cancer survival in relation to $S 6 K 2$ and/4EBP1 $m R N A$, in the van de Vijver, Karolinska and Uppsala patient cohorts respectively; IGF1R and/or IGF2R and/or HER2 high (a, c, e); IGF1R and IGF2R and HER2 low (b, $d, f)$. 
For S6K1 mRNA, no prognostic value in the HER2/IGFR positive patient group was seen. IGF1R and IGF2R are thought to have opposite roles in IGFR signalling, but addition of IGF2R to the IGF1R and HER2 positive subgroup improved the prognostic value of S6K2/4EBP1, through reasons that need to be further evaluated. Likely, autocrine or paracrine growth factor signalling is still essential for the activation of intracellular signalling via S6K2/4EBP1. S6K1, in turn, may inhibit RTK signalling via the feedback regulation of IRS1.

Differences between S6K2 and 4EBP1 profiles could also be seen. One interesting finding was the strong inverse correlation between 4EBP1 and ESR1 mRNA, encoding the ER $\alpha$ (Study 4). When restricting the analysis to ER negative tumours, 4EBP1 was positively associated with mRNA expression of oestrogen-related receptor alpha (ESRRA). ESRRA was identified as a result of its close homology to the ER, and is preferably expressed in ER negative breast cancers $[305,306]$. It is considered an orphan receptor, and its activity has been shown regulated by the PI3K/AKT signalling pathway. The 4EBP1 correlation to ESRRA could also be confirmed in two additional datasets, preferably in the ER negative subcohort. These results may suggest an alternative signalling through ESRRA, independent of the ER, in tumours expressing high levels of 4EBP1.

Analyses of mRNA levels in tumour tissue in general reflect the combined mRNA expression in several types of cells in addition to the tumour cells, including stromal and normal epithelial cells. The mRNA-levels are in turn a result of a complex gene regulation on multiple levels, intracellular as well as determined by a cross-talk between different cells. It has been shown that cancer stem cells may go through a metamorphosis, allowing a differentiation to several cell types [21], suggesting that all cells in a tumour may stem from the same progenitor. In the light of this, analysing the combined mRNA expression of tumours may be defendable.

\section{Intracellular localisation and expression levels of S6K1, S6K2 and $4 E B P 1$ protein determine associations with clinicopathological factors and predictive role for endocrine treatment benefit}

Studies on gene copy number and mRNA levels of the mTOR targets S6K1, S6K2 and 4EBP1 have resulted in data pointing towards a clinical potential of these factors in breast cancer, and indicated partly divergent roles for the homologous S6 kinases in this context. The relationship between gene copy number/mRNA and corresponding protein expression levels and functions is debated. A recent review on this issue conclude that the abundance of mRNA in general highly reflects the ability to detect protein expression in cells [307]. Unfortunately, we have not been able to investigate the possible relation between gene/mRNA levels and its corresponding protein expression for S6K1, S6K2 and 4EBP1. However, access to material from the large randomised Stockholm 3 trial allowed us to explore the clinical potential of these factors on the protein level.

S6K1, S6K2 and 4EBP1 protein expression could be ubiquitously detected in the nucleus and cytoplasm of breast tumours (Study 1, 3 and [249]. Furthermore, S6K phosphorylated at the mTORC1 activation site T389 (pS6K) and 4EBP1 phosphorylated at S65 were evaluated in the material (Study 3 and [249]). Earlier implicated associations between S6K1, S6K2 and 4EBP1 and clinicopathological factors as well as prognosis seem to be dependent on the cellular localisation of the protein. In line 
with the findings for RPS6KB1 amplification, cytoplasmic S6K1 (S6K1cyt) was also associated with HER2 positivity. S6K1 in the nucleus (S6K1nucleus) could be weakly connected to both HER2 and ER positivity. On the contrary, S6K2, especially nuclear, was strongly correlated to ER and PgR expression. Nuclear pS6K was also weakly associated to ER. Cytoplasmic 4EBP1 and p4EBP1 was inversely connected to ER-positivity, as earlier shown for 4EBP1 mRNA, and positively associated to high grade. Table 7 shows the correlations of S6K and 4EBP1 to breast cancer subtypes.

$\mathrm{S} 6 \mathrm{~K} 1$ in the cytoplasm was associated with high grade and a poor prognosis, whereas no prognostic value was evident for S6K2 or pS6K, regardless of cellular location (Study 1 and [249]. High cytoplasmic levels of p4EBP1 have been shown to predict a poor prognosis in several cancer types [189, 190, 294, 295] and this could also be confirmed in the present study (Figure 26). Interestingly, 4EBP1 independent of phosphorylation status was also indicated to associate with prognosis, and the variable 4EBP1cytoplasm>nucleus, indicating higher cytoplasmic than nuclear levels of 4EBP1, was significantly predictive of an adverse outcome among systemically untreated breast cancer patients (Figure 26).

Table 7 Protein expression of S6K1, S6K2, pS6K, 4EBP1 and p4EBP1 in relation to Breast Cancer Subtypes, assessed by Spearman's Rank order correlation. Breast cancer subtypes in Stockholm 3 were defined according to St Gallen 2011 [9] as follows: Luminal A (ER+ and/or PgR+, Her2-, grade I-II), Luminal B1 (ER+ and/or PR+, HER2-, grade III), Luminal B2 (ER+ and/or PR+, HER2+), HER2-like (ER- and PR- and HER2+), and Basal-like (ER- and PR- and HER2-) (R-values for $p<0.01$ highlighted, positive correlations in grey).

Breast cancer subtype

\begin{tabular}{|c|c|c|c|c|c|}
\hline & $\begin{array}{l}\text { Luminal A } \\
\mathrm{n}=457(63.0 \%)\end{array}$ & $\begin{array}{l}\text { Luminal B1 } \\
\mathrm{n}=54(7.4 \%)\end{array}$ & $\begin{array}{l}\text { Luminal B2 } \\
\mathrm{n}=37(5.1 \%)\end{array}$ & $\begin{array}{l}\text { HER2-like } \\
\mathrm{n}=55(7.6 \%)\end{array}$ & $\begin{array}{l}\text { Triple negative } \\
\mathrm{n}=123(16.9 \%)\end{array}$ \\
\hline S6K1cyt & -0.09 & 0.09 & 0.16 & 0.04 & -0.07 \\
\hline S6K1nucleus & 0.01 & 0.02 & 0.17 & 0.00 & -0.12 \\
\hline S6K2cyt & 0.09 & -0.04 & 0.02 & -0.02 & -0.08 \\
\hline S6K2nucleus & 0.32 & -0.16 & -0.04 & -0.08 & -0.22 \\
\hline pS6Kcyt & -0.01 & 0.05 & 0.00 & -0.01 & -0.03 \\
\hline pS6Knисleus & 0.07 & 0.00 & 0.06 & -0.03 & -0.13 \\
\hline 4EBP1cyt & -0.23 & 0.17 & 0.06 & 0.10 & 0.04 \\
\hline 4EBPInucleus & -0.11 & 0.11 & 0.04 & 0.03 & 0.01 \\
\hline p4EBPIcyt & -0.28 & 0.15 & 0.08 & 0.06 & 0.13 \\
\hline p4EBP1nucleus & 0.08 & -0.05 & -0.09 & -0.04 & 0.01 \\
\hline
\end{tabular}



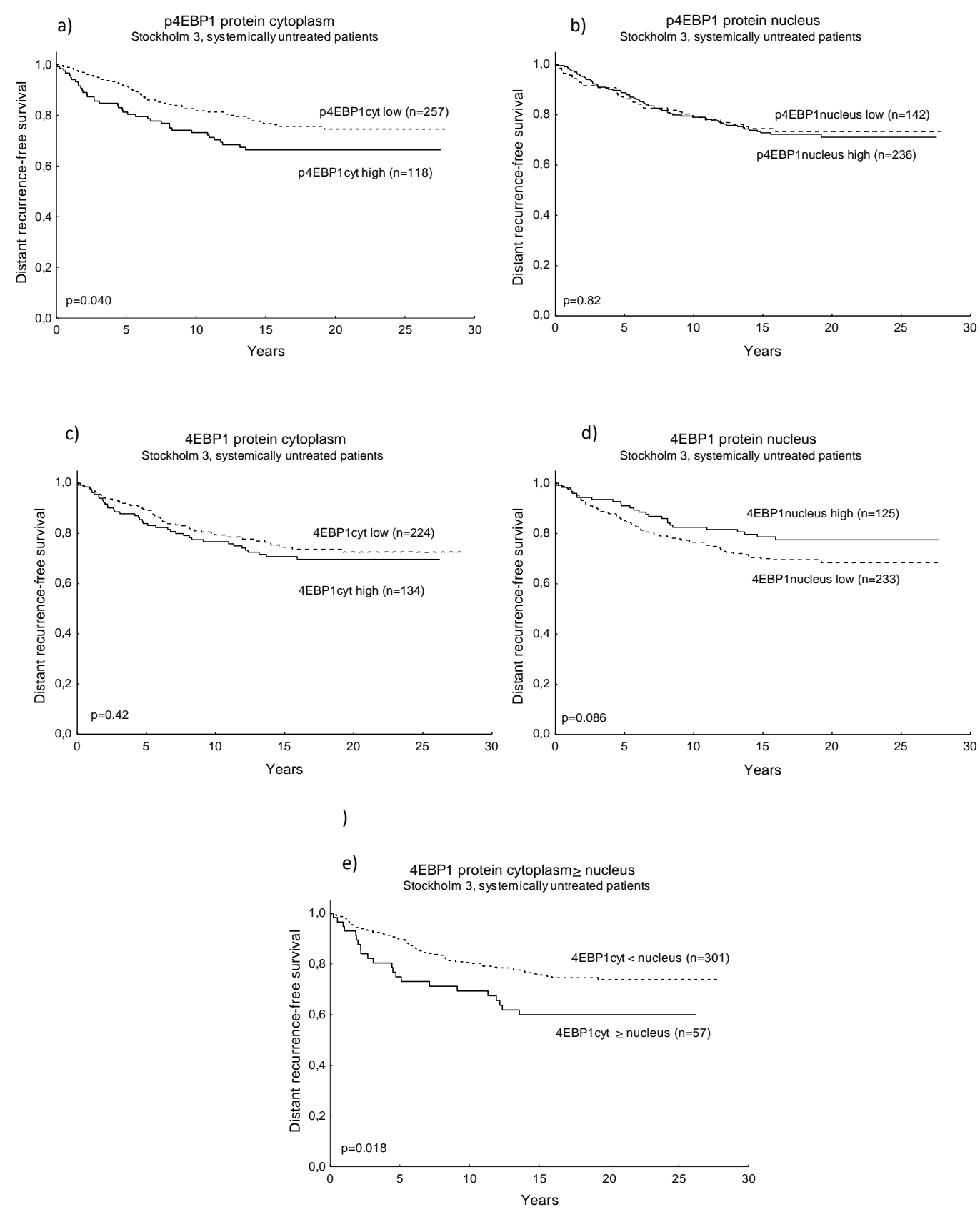

Figure 26 Distant recurrence-free survival among systemically untreated patients in the Stockholm 3 cohort, in relation to $p 4 E B P 1$ cytoplasmic protein (a), p4EBP1 nuclear protein (b), 4EBP1 cytoplasmic protein (c), 4EBP1 nuclear protein (d), 4EBP1cyt $\geq$ nucleus (e). 
New clinical markers predicting the benefit from different treatments are essential to improve the possibilities to further individualised therapies. As mentioned above, RPS6KB2 gene gain was shown able to predict benefit from tamoxifen treatment in the studied cohort. In line with this, high levels of nuclear S6K2 protein was also associated with tamoxifen benefit, although restricted to the ER/PgR positive subgroup (Figure 27). On the other hand, in the ER positive/PgR negative cohort, nuclear S6K2 with borderline significance predicted a poor benefit from tamoxifen. Also nuclear S6K1 was associated with less tamoxifen benefit, and this reached significance for the variable S6K1nucleus $>$ cyt in the ER positive subgroup. A similar trend was seen for $\mathrm{pS6K}$, but here the variable $\mathrm{pS} 6 \mathrm{Kcyt}$ and/or nucleus was needed to reach significance in the analysis [249]. The associations of S6K1, S6K2 and pS6K with endocrine resistance, for S6K2 restricted to the ER positive/PgR negative subgroup, allow for the speculation that ER signalling in this subgroup may be driven in a hormone-independent manner, via cross-talk to intracellular signalling pathways including mTOR/S6K. In vitro studies have shown that S6K1 may phosphorylate ER at S167, resulting in ligand-independent activation of the receptor [165]. Accordingly, nuclear pS6K, S6K1 as well as S6K2 was correlated with pER_S167, whereas the latter was also associated with pER_S305 in the material (unpublished data).

The correlation between S6K2 and 4EBP1 could be confirmed at the protein level, for both the cytoplasmic and nuclear compartments. In addition S6K1 and 4EBP1 was associated, and pS6K correlated to S6K1 and S6K2 in both compartments. Cytoplasmic 4EBP1 was predictive of a poor benefit from tamoxifen in the ER positive/PgR positive subgroup (Figure 27) and a similar trend could be seen for p4EBP1. 

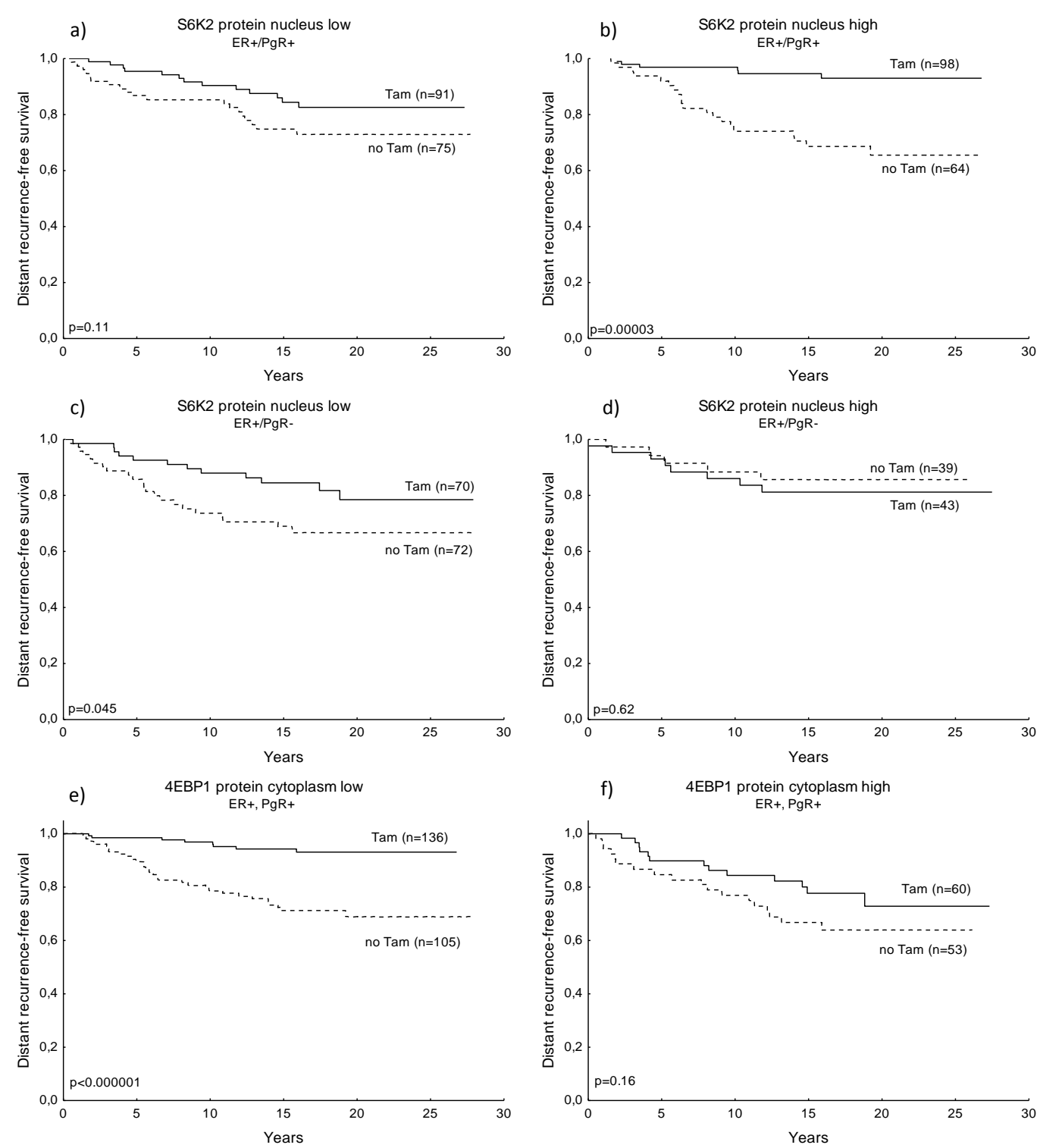

Figure 27 Distant recurrence-free survival among patients in the Stockholm 3 cohort treated or not treated with tamoxifen, in the groups of patients with ER-positive tumours harbouring: low S6K2nucleus, PgR+ (a), high S6K2nucleus, PgR+ (b), low S6K2nucleus, PgR- (c), high S6K2nucleus, $P g R-(d)$, low 4EBPlcyt, PgR+ (e), high 4EBPlcyt, PgR+. Tests for interaction: PgR+ S6K2nucleus vs. tam $p=0.034$, PgR-S6K2nucleus vs. tam: $p=0.13$, PgR+ 4EBP1cytoplasm: $p=0.034$. 
4EBP1 is regulated by phosphorylation at multiple sites, and the roles for the different sites are not totally established. The 4EBP1 antibody was raised towards a sequence surrounding S112, thus at the very $\mathrm{C}$-terminus of 4EBP1, and recognises both unphosphorylated as well as 4EBP1 phosphorylated at different sites. In addition, the 4EBP1 and p4EBP1_S65 stainings were highly correlated, especially for the cytoplasmic pools of the proteins, indicating that to some extent, the same proteins are detected. This may also reflect that an increase in total protein expression often is accompanied with an increased phosphorylation and activation of the proteins. Therefore, it is possible that 4EBP1 activation (by foremost mTOR) may be the reason behind its role in endocrine resistance. Interestingly, in a recent publication, both phosphorylated and total 4EBP1 was related to a poor outcome among patients with ER positive breast cancers, treated with tamoxifen [308], in keeping with our findings. In that study, protein expression was determined by reverse-phase protein arrays, ruling out the possibility to distinguish between cytoplasmic and nuclear expression.

In the present study, the endocrine treatment predictive value for 4EBP1, as well as S6K2, was especially evident in the ER/PgR expressing subgroup. In addition, the prognostic significance of 4EBP1 was most prominent in combination with PgR expression, suggesting a possible cross-talk to nuclear receptors. The role of progesterone signalling in breast cancer remains controversial. In general, circulating progesterone is considered as a risk factor for breast cancer development by promoting cellular proliferation. However, in primary breast cancer, PgR expression is associated with differentiated, less aggressive tumours and a favourable prognosis [31]. Upregulation of the IGF/PI3K/AKT/mTOR pathway is one suggested mechanism behind PgR downregulation in breast cancer, despite a functional ER. In agreement, our study showed an inverse association between S6K2/4EBP1 and PgR mRNA levels, in the three available cohorts. Furthermore, the gene encoding $\mathrm{PgR}$ is located at the proximal part of the 11q chromosomal arm, which is commonly deleted in 11q13/8p12 amplified tumours (Study 2). However, 4EBP1 was recently described as a possible target gene for PgR[309], suggesting the presence of a negative feedback loop downregulating PgR after growth factor pathway stimulation. The function of $\mathrm{PgR}$ can be regulated by receptor phosphorylation at multiple sites, through growth factor receptor signalling pathways, and a subpopulation of cytoplasmic PgR has also been shown able to activate kinase cascades, including PI3K/AKT [31]. It is tempting to speculate that a coordinated expression of $\mathrm{PgR}$ and cytoplasmic growth signalling factors including S6K2/4EBP1 may facilitate the proliferative and oncogenic role of PgR, promoting tumour progression and therapy resistance. In addition, PgR may in the long run be downregulated through $\mathrm{PI} 3 \mathrm{~K} / \mathrm{AKT} / \mathrm{mTOR}$ pathway stimulation and subsequent aberrant ER signalling, leading to acquired endocrine resistance among patients with initially ER/PgR positive breast cancers.

Altogether, the expression levels and possibly the phosphorylation status of the mTOR effectors S6K1, S6K2 and 4EBP1 may be potential candidates as markers for prediction of benefit from tamoxifen treatment. In combination with their role in prognosis, or with the addition of other prognostic markers, these factors may consequently enable a more secure judgement on whether tamoxifen therapy is needed, when this therapy only may be useful, or when a combination of endocrine treatment with other therapies may be essential. A similar role for the prediction of other endocrine therapies may be conceivable, however, this needs further investigation. Studies have also shown several other factors, among others in the PI3K/AKT pathway, to be useful in the prediction of endocrine treatment benefit [286, 310,311]. Meta-studies comparing the usefulness, functionality and reproducibility of different markers, are needed to pin-point the optimal factor or combination of markers for this important purpose. 


\section{Structural comparison and in vitro silencing of S6K1 and S6K2 suggest a possibility to develop isoform specific inhibitors}

The presented findings indicate clinically relevant differences between the mTOR effectors S6K1 and S6K2, and that amplification and overexpression may be present in partly different breast cancer subtypes. A recent review also highlights these indications [118]. In the light of this, it may be conceivable that isoform-specific targeting could be useful, allowing further tumour-specific therapies. Consequently, a study was performed using RNA interference for in vitro silencing of S6K1 and S6K2 separate or along, with subsequent transcriptional analysis to explore their effects on global mRNA expression profiles (Study 4). The breast cancer cell line ZR751 was used as a model due to the relatively high expression levels of both S6K1 and S6K2 ([240] and unpublished observations). Data indicated that silencing of S6K1 and S6K2 results in a partly compensatory upregulation of the other isoform, respectively. A feedback upregulation of mTOR, Raptor and Rictor in the two mTOR-complexes was evident, especially during simultaneously silencing of S6K1 and S6K2. Also an upregulation of p90 S6 kinases could be seen, likely compensating for the loss in rpS6 phosphorylation.

Isoform-specific silencing of S6K1 and S6K2, respectively, also resulted in significant differences in global expression profiles. The genes upregulated by S6K1siRNA, but not S6K2siRNA, included EIF4E2, CCND1, FGFR1, INSRR and the mitogen activated kinases MAP3K4 and MAPK3. Downregulated genes after S6K1 knock-down included EIF4EBP1 and EIF4G2, as well as the adaptor $G A B 3$. Enriched upregulated genes were coupled to membrane-bounded organelle, targets of miR144 and the transcription factors USF and CDPCR1.

S6K2 silencing resulted in upregulation of genes enriched in several cellular processes. These represented cell cycle/mitosis; among others CCNE1, CCNE2, CCNG2, CCNL1, CCNT1, CDK12 and $C D K N 3$, prostate cancer; including the androgen receptor (AR), metabolism as well as targets of the transcription factors $E 2 F, A P 2, S P 1, E T F$ and $P a x 3$. Other upregulated genes upon S6K2 knock-down included the PI3K/AKT associated genes IGF2R, PTEN, PIK3R3, EIF2AK2 and EIF5B, the oncogenes $A B L 1$ and MDM2, and the tumour suppressor candidates DGDHS, TSSC1 and TUSC3. Downregulated genes after S6K2siRNA included genes in ion and monoamine transport as well as ClassA1 Rhodopsin-like receptors. Downregulated genes of note included ABL2 and PTPN22. Eight genes were downregulated with S6K1 knock-down, but upregulated after S6K2 silencing (Study 4). In line with previous data, these results support the indices of compensatory as well as specific roles for S6K1 and S6K2. We and others ([222] and unpublished data) have noticed that the compensatory upregulation of pAKT via mTORC1/S6K1 silencing, seems not to occur after S6K2 silencing, further supporting this. Additional studies are needed to explore the effect of S6K1 and S6K2 silencing/inhibition on compensatory mechanisms on the protein level, as well as possible antitumourigenic effects under different circumstances in vitro and in vivo.

Whole-genome profiling of S6K1 and S6K2 high tumours, as well as after in vitro knock-down of the two kinases both resulted in significant differences, suggesting that isoform specific targeting of S6K1 or S6K2 may be valuable to allow further individualised breast cancer treatment regimens. The development of specific kinase inhibitors for therapeutic purposes is desirable [312]. However, the conserved homology between kinases has obstructed this process. The high similarity between the S6K1 and S6K2 primary structures allowed us to use the crystal structures of S6K1 for in silico 
predictions of the S6K2 kinase structure (Study 4). The resulting models point towards some essential differences in the ATP-binding site of the two kinases, possibly allowing design of isoform-specific inhibitors (Figure 28).

Initially, to evaluate structural differences between S6K1 and S6K2, the primary structures were aligned using BLAST. S6K1 and S6K2 are both composed of 15 exons, were 1-14 are homologous, resulting from gene duplication. In contrast, exon 15 is different between the two kinases; S6K1 has a C-terminal PDZ-binding motif whereas S6K2 contains a proline-rich domain (Study 4). The amino acid sequences in the domains are highly similar. The residues in the active site, substrate binding site and hydrophobic motif are identical between the two kinases. The ATP binding site contains one amino acid shift, S6K1-S6K2: Y151C (S6K1 residue numbering), resulting in a slightly different size of the ATP-binding pockets between the two kinases (Figure 28). The corresponding residue in other members of the AGC family are commonly aromatic (Tyrosine or Phenylalanine) and to our knowledge, S6K2 and the 35\% homologous SGK494 are the only members of the AGC family of kinases to have a non-aromatic residue at this site, facilitating development of S6K2-specific inhibitors. Two amino acid shifts are also present in the activation loop S6K1-S6K2: D223E and T225A, possibly allowing differences in the mechanisms of kinase activation

The S6K1 kinase structure bound to staurosporin (Figure 28 a) showed relatively low electron density in the $\alpha \mathrm{C}$-helix as well as the activation loop both regulating kinase activity, indicating highly dynamic structures, although the phosphorylation at T229 partly stabilised the structure. When S6K2 was modelled against this structure, the $\alpha \mathrm{C}$-helix was assumed to be even shorter in S6K2 (residues R120-S125) than in S6K1 (residues A111-E126) (Figure 28 b), possibly as a result of differences in neighbouring amino acids, leading to an even more dynamic structure of S6K2.

The S6K1 kinase structure bound to the specific inhibitor PF-4708671 (Figure 28 c) showed significantly higher electron density in the $\alpha \mathrm{C}$-helix compared to the staurosporin bound structure, suggesting that the inhibitor stabilises the kinase domain in a non-activating conformation. Consequently, when using this structure as a template, also the S6K2 showed a longer, apparently less dynamic, $\alpha \mathrm{C}$-helix (Figure $28 \mathrm{~d}$ ). Altogether, the structure comparisons between S6K1 and S6K2 indicate highly similar secondary and tertiary structures, but also dynamic structures. The similarities are likely more pronounced than in reality and the modelled structures are clearly biased towards the used template. It is for instance noteworthy that the two different S6K1 structures that were used as templates deviate more from each other with an RMSD of $1.25 \AA$ (backbone), $1.35 \AA$ (all heavy atoms) than the respective templates and the models. However, even if the structures of S6K1 and S6K2 kinase domains are as similar as the models suggest, there are differences that can be exploited for development of S6K2 specific inhibitors. The unique presence of the amino acid Cysteine in S6K2 at a position occupied by Tyrosine in S6K1, creates a significantly different environment in the ATPbinding domain, suggesting an opportunity for future development of isoform-specific inhibitors.

This study sheds further light on downstream mTOR signalling in breast cancer, supporting that S6K1 and $\mathrm{S} 6 \mathrm{~K} 2$ signalling may in part possess different roles in tumourigenesis. A structural comparison of the S6K1 and S6K2 kinase domains revealed significant differences that could be of importance for differences in regulation and function of the two kinases and also useful for future development of isoform-specific inhibitors. At the global tissue level, the expressions of S6K1 and S6K2 have been shown inversely correlated [118]. S6K1 are ubiquitously expressed, whereas the levels of S6K2 vary between tissue and the protein is mainly found in the central nervous system, lungs and gastrointestinal tracts. S6K2 levels in tumour tissue are in general high compared with corresponding 
normal tissue. The emerging different roles of S6K1 and S6K2 suggest that specific targeting of either isoform may be valuable in different tumour subtypes, and in comparison to present day's mTOR inhibitors, further promote individualised therapies. 

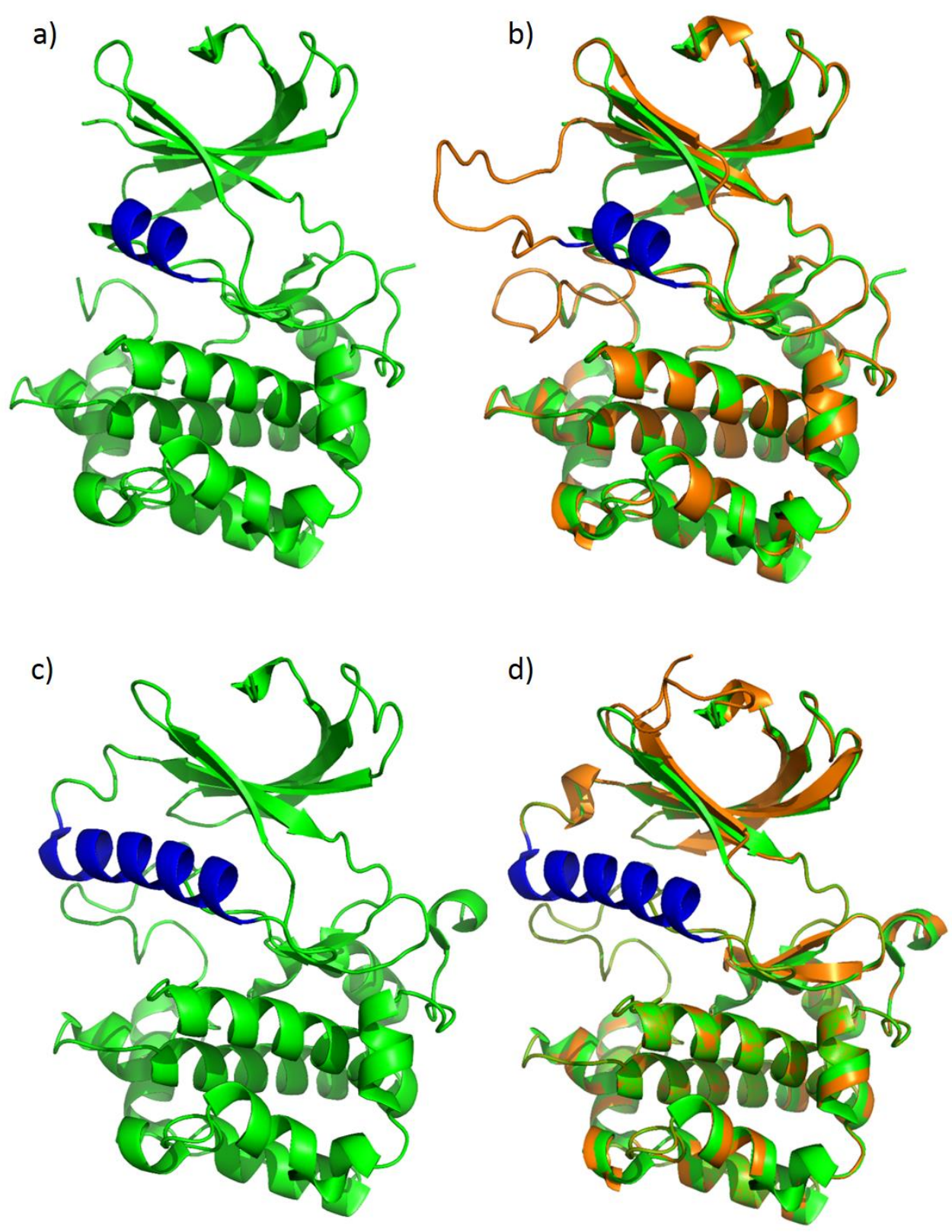

Figure 28 Structural comparison of S6K1 PDB ID $3 A 62$ (a) and S6K1 PDB ID 4L3J (c) and overlaid with the corresponding $S 6 K 2$ homology models (b) and (d). 


\section{CONCLUSIONS}

In summary, the results of the present project suggest that:

- The mTOR effector S6K2 is a new candidate oncogene in the 11q13 amplicon in breast cancer (Study 1).

- S6K2/4EBP1 coamplification and mRNA coexpression is associated with a poor outcome, indicating a synergy between mTOR targets (Study 2 and 3).

- Whole-genome profiles of S6K2 and 4EBP1 positive tumours are highly overlapping and associated with cell cycle regulators and IGF signalling, indicating different roles of S6K1 and S6K2 (Study 4)

- Intracellular localisation and expression levels of S6K1, S6K2 and 4EBP1 determines associations with clinicopathological factors and indicate possible predictive roles for endocrine treatment benefit (Study 1 and 3)

- Structural comparison and in vitro silencing of S6K1 and S6K2 suggest a possibility to develop isoform specific inhibitors, which may facilitate further individualised therapies for breast cancer (Study 4). 


\section{PERSPECTIVES}

"A good gene in one time and place may be a bad gene in another time or place"

This quotation proposed by Woolhouse et al. (2002) in the context of spread of viruses, may actually be well applied to conclude the riddle of cancer. In one way or another, this may explain the main questions; why does cancer arise? How can it be prevented? And, when developed, how can it be treated? No doubt, cancer arises as a result of a sustained long-term complex interplay between the outer and inner environment and an extensive number of genetic and epigenetic factors, throughout all tissues and cells of the body. These comprehensive interactions also determine the faith of the malignant progression, as well as the quality of response to applied treatment, shortly and long-term. To distinguish the underlying patterns and mechanisms explaining which gene is good or bad in which time and place would possibly be essential to find the answers.

During the last years, cancer research has moved the first, trembling steps towards this future utopia. For a number of cancer diseases, specific environmental features have been identified as risk factors, to a lower or higher degree, for malignant development. Also, certain genetic variants have been shown predisposing for cancer, or protective against the disease. The extensive numbers of alterations found in tumours have been proposed to result in a few underlying capabilities, or "hallmarks" of cancer. For breast cancer, whole genome-studies have been able to distinguish a limited number of tumour subtypes. These have in turn been possible to relate to different states of the normal gland development, giving a further dimension of breast cancer development.

An integration of the comprehensive amount of present data resulting from studies in different fields of cancer research and a large common effort in objective analysis, is certainly needed in continuation towards the utopia proposed by Hanahan and Weinberg that cancer biology will develop into a logical science where the complexities of the disease can be described in a few, underlying principles, finally allowing prevention of malignant development, patients specific diagnosis and tailored treatment strategies. Our hope is, that in the light of other findings, this present study may be able to be a contribution in some way to this comprehensive puzzle and its future solution. 


\section{ABBREVIATIONS}

The lists of abbreviations contains two parts, the first one including genes and gene products which are not spelt out in the text, and a second part of other scientific terms, which are as normally explained with full names in the text.

\section{Genes and gene products}

4EBP1

$\mathrm{ABL}$

ACTB

AIB1

AKT

ALDH1

ALK

AMPK

AP1

Apaf1

AR

AREG

Axl

BCL1

BCL2

BRCA 1 and 2

C11 orf30

$\mathrm{C} / \mathrm{EBP} \beta$.

$\mathrm{C} 1, \mathrm{C} 2$

CBP

$\mathrm{CCN}$

CCNA

$\mathrm{CCNB}$

CCND1 (BCL1, PRAD1)

$\mathrm{CCT} \beta$

$\mathrm{CD} 24$

$\mathrm{CD} 44$

CDC42, CDC25B, CDC20

CDH1

Cdk

CENPA

CHEK2

CK2

c-Myc (Myc)

CREM $\tau$

CTTN (EMS1)
EIF4E-binding protein 1

Abelson tyrosine-protein kinase

beta-actin

amplified in breast cancer 1

protein kinase $\mathrm{B} / \mathrm{v}$-akt murine thymoma viral oncogene homologue

aldehyde dehydrogenase

Anaplastic lymphoma kinase

AMP-activated protein kinase

activator protein 1(transcription factor)

apoptosis activating factor 1

androgen receptor

amphiregulin

tyrosine-protein kinase receptor UFO

B-cell lymphoma 1 (CCND1)

B-cell lymphoma 2

Breast Cancer gene 1 and 2.

chr 11 open reading frame 30, encoding the EMSY protein

CCAAT/ enhancer transcription factor

PKC activating domains 1 and 2

cap binding protein

cyclin gene

cyclin A

cyclin B gene

cyclin D1 gene

chaperonin containing $\mathrm{T}$-complex protein

cluster of differentiation 24

cluster of differentiation 44

cell division cycle

cadherin 1 , e-cadherin

cyclin-dependent kinase

Centromere protein A

CHK2 checkpoint homolog

casein kinase 2

cellular myocytosis gene

cAMP responsive element modulator

cortactin 


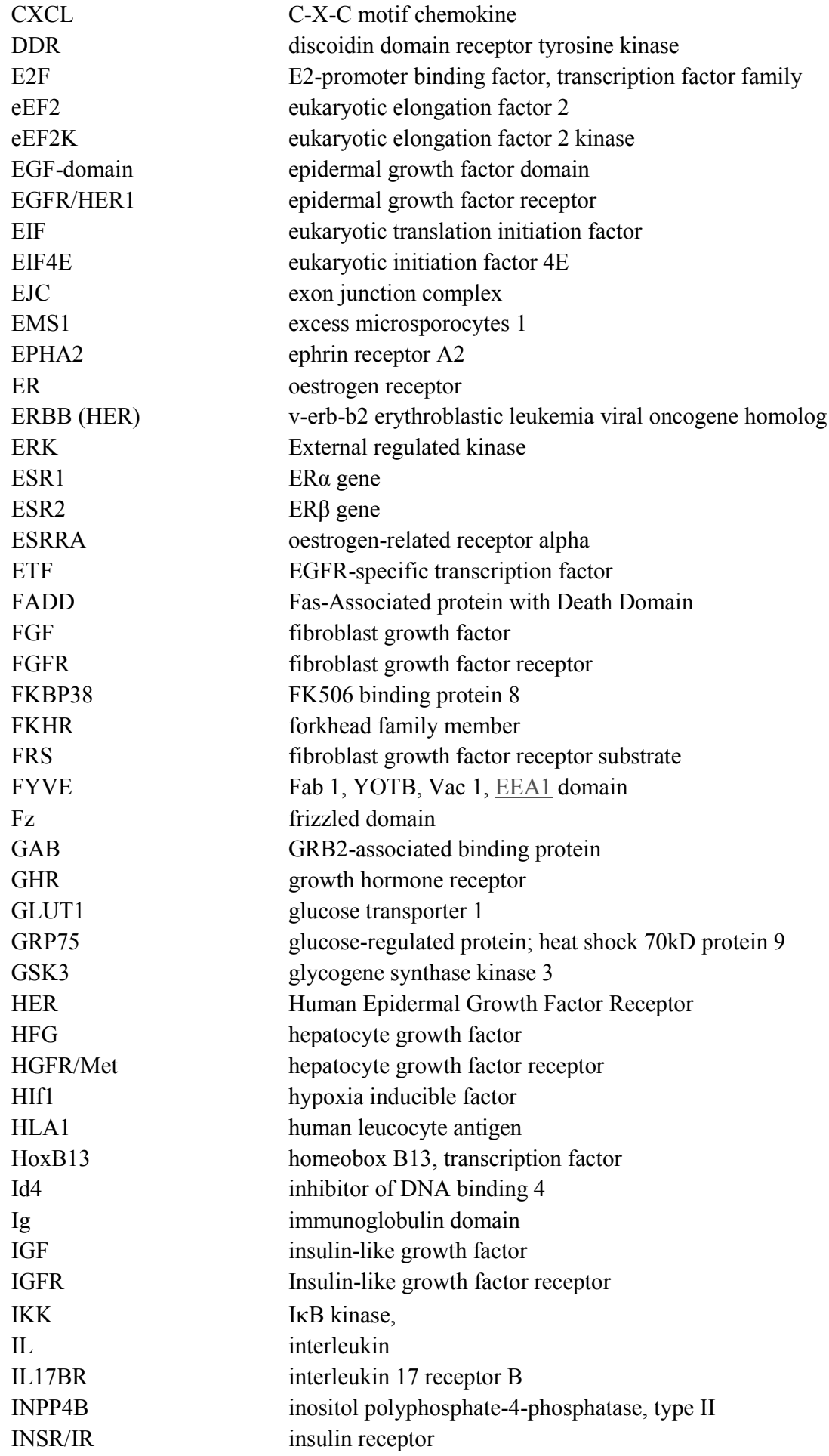




\begin{tabular}{|c|c|}
\hline INSRR & insulin-related receptor 1 \\
\hline IRS & insulin-receptor substrate \\
\hline JNK & c-Jun N-terminal kinase \\
\hline Ki-67 & Antigen identified by monoclonal antibody Ki-67 \\
\hline Ldla & Low Density Lipoprotein Receptor Class A domain \\
\hline LKB1 (STK11) & Serine/threonine kinase 11 \\
\hline LMR & Lemur \\
\hline Mam & meprin/A5-protein/PTPmu \\
\hline MAPK & mitogen activated protein kinase \\
\hline MAPKAP & Target of rapamycin complex 2 subunit \\
\hline MDM2 & Mouse double minute 2 homolog \\
\hline MICB & MHC class I polypeptide-related sequence B \\
\hline mLST8 & MTOR associated protein, lethal with SEC13 protein 8 homolog \\
\hline MMP & matrix metalloprotease \\
\hline mTOR & mammalian/mechanistic target of rapamycin \\
\hline mTORC & mTOR complex \\
\hline Musk & Muscle-Specific Kinase \\
\hline MYEOV & myeloma overexpressed \\
\hline p4EBP1 & phosphorylated 4EBP1 \\
\hline P53 & Tumour protein $53(\mathrm{p} 53)$ \\
\hline PAI-1 & plasminogen activator inhibitor type 1 \\
\hline PAK1 & p21-activated kinase 1 \\
\hline PAPB1 & polyA binding protein complex \\
\hline PAX & paired box transcription factor \\
\hline PCNA & proliferating cell nuclear antigen \\
\hline PDCD4 & programmed cell death 4 \\
\hline PDK1 & phosphoinositide dependent protein kinase 1 \\
\hline PDZ & $\begin{array}{l}\text { post synaptic density protein (PSD95), Drosophila disc large tumor } \\
\text { suppressor (Dlg1), and zonula occludens-1 protein (zo-1) domain }\end{array}$ \\
\hline PGK1 & Phosphoglycerate kinase 1 \\
\hline PgR & progesterone receptor \\
\hline $\mathrm{PH}$ & pleckstrin homology \\
\hline PHLPP & PH domain and Leucine rich repeat Protein Phosphatase \\
\hline PI3K & phosphatidyl inositol 3-kinase \\
\hline PIK3C & PI3K subunit p1 10 genes \\
\hline PIK3R & PI3K subunit $\mathrm{p} 85$ genes \\
\hline PIKK & phosphoinositide kinase related kinase \\
\hline $\mathrm{PIP}_{2}$ & phosphatidylinositol-4,5-bisphosphate \\
\hline $\mathrm{PIP}_{3}$ & phosphatidylinositol-3,4,5,-trisphosphate \\
\hline PKA & protein kinase A \\
\hline PKB (AKT) & protein kinase B \\
\hline PP2A & protein phosphatase $2 \mathrm{~A}$ \\
\hline PPAPDC1B & Phosphatidic acid phosphatase type 2 domain-containing protein $1 \mathrm{~B}$ \\
\hline PPFIA1 & $\begin{array}{l}\text { Protein Tyrosine Phosphatase, Receptor Type, F Polypeptide (PTPRF), } \\
\text { Interacting Protein (Liprin), Alpha } 1\end{array}$ \\
\hline РPH3 & phosphohistone $\mathrm{H} 3$ \\
\hline PPIA & peptidylprolyl isomerase A (cyclophilin A) \\
\hline
\end{tabular}




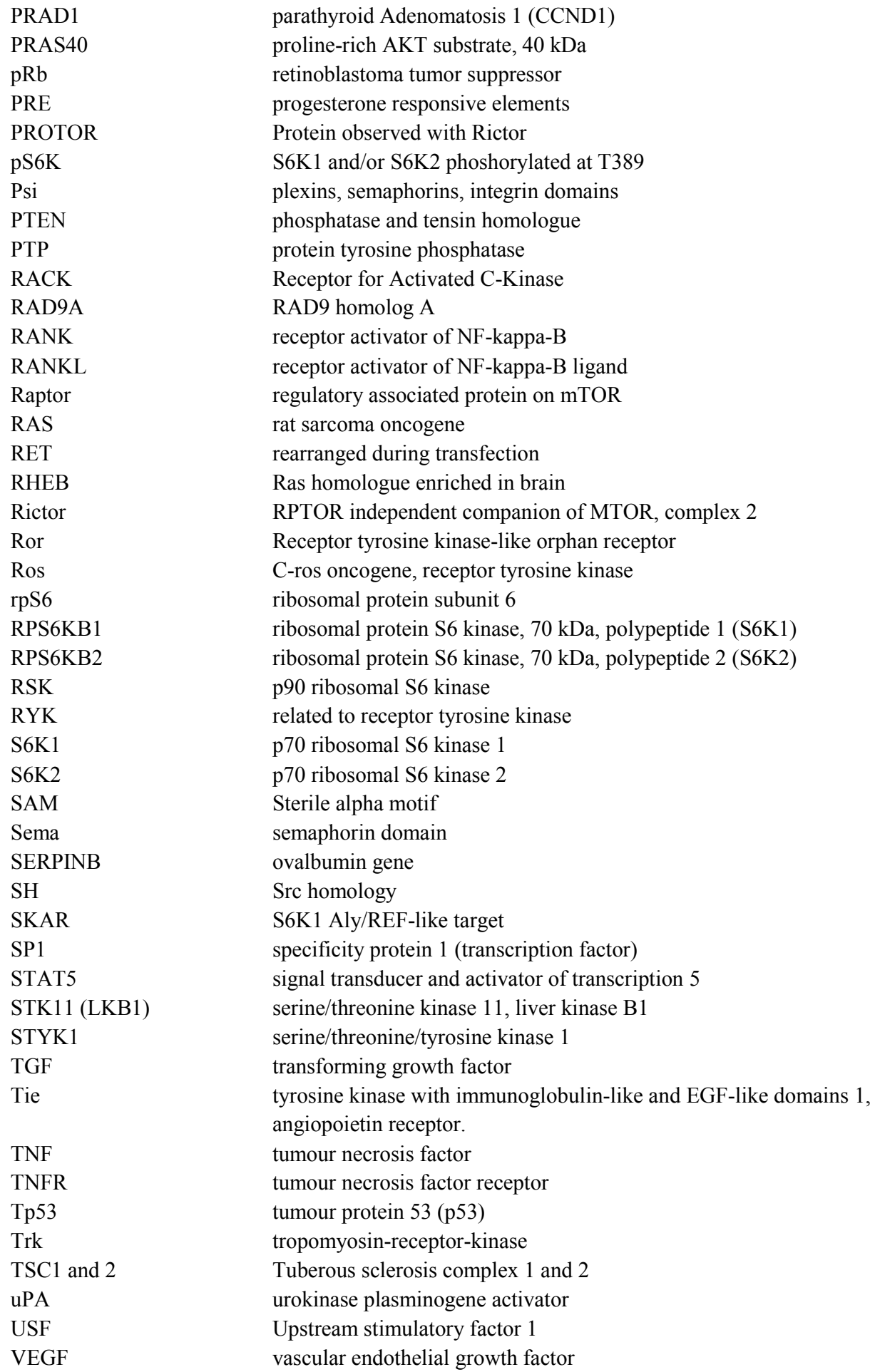


WIF

Wnt

WW

YWTD

YY1

ZEB
Wnt-inhibitory factor

wingless-type MMTV integration site family

Trp-Trp repeat motif domain

Tyr-Trp-Thr-Asp repeat

Yin Yang 1

zinc finger E-box binding homeobox 1 


\section{Scientific terms}

AGC cAMP-dependent, cGMP dependent and protein kinase $\mathrm{C}$ family

ASCO American society of clinical oncology

ATCC American Type Culture Collection

BCS breast cancer survival

BLAST Basic local alignment search tool

BMI body mass index

BRLMM Bayesian Robust Linear Model with Mahalanobis distance classifier

CAF cancer associated fibroblast

cDNA complementary DNA

CI confidence interval

CISH chromogenic in situ hybridisation

CMF cyclophosphamide methotrexate 5-fluorouracil chemotherapy

CR C-terminal regulatory region

CSC cancer stem cell

$\mathrm{Ct} \quad$ threshold cycle

CTC circulating tumour cell

DAB 3.3'-diaminobenzidine hydrochloride

DCIS ductal carcinoma in situ

DDBJ DNA database of Japan

DM double minutes

DRFS distant recurrence-free survival

E2 oestradiol

ECM extracellular matrix

EIA enzyme immunoassay

EMBL-EBI European Bioinformatics Institute

EMT epithelial to mesenchymal transition

ERE oestrogen-responsive elements

FASTA fast all

FEC fluorouracil/5FU, epirubicin cyclophosphamide

FFPE formalin fixated paraffin embedded

FISH fluorescent in situ hybridization

G1 gap1 phase of cell cycle

G2 gap2 phase of cell cycle

gDNA genomic DNA

GEO gene expression omnibus

GISTIC genomic identification of significant targets in cancer

GnRH gonadotropin-releasing hormone

GO gene ontology

Gy Gray (unit)

HeLa cervix carcinoma cell line, from the patient Henrietta Lacks

HMM Hidden Markov Model

hnRNP heterogeneous ribonucleoprotein

HNSCC head and neck squamous cell carcinoma

HR hazard ratio

HSR Homogeneously staining regions 


$\begin{array}{ll}\text { ICM } & \text { Internal Coordinate Mechanics (software) } \\ \text { IDC } & \text { Invasive Ductal Carcinoma } \\ \text { IHC } & \text { immunohistochemistry } \\ \text { ILC } & \text { Invasive Lobular Carcinomas } \\ \text { IRES } & \text { internal ribosomal entry sites } \\ \text { KD } & \text { kinase domain } \\ \text { kDa } & \text { kilodalton (unit) } \\ \text { KE } & \text { kinase extension domain } \\ \text { LCIS } & \text { Lobular carcinoma in situ } \\ \text { LRFS } & \text { local recurrence-free survival } \\ \text { Mb } & \text { megabase (million base pairs) } \\ \text { MCF7 } & \text { Michigan Cancer Foundation-7, breast cancer cell line } \\ \text { MEF } & \text { mouse embryonic fibroblast } \\ \text { MIAME } & \text { minimum information about a microarray experiment } \\ \text { miR } & \text { microRNA } \\ \text { MM } & \text { mismatch } \\ \text { M-phase } & \text { mitotic phase of cell cycle } \\ \text { NCBI } & \text { National Center for Biotechnology } \\ \text { NLM } & \text { National Library of Medicine } \\ \text { NLS } & \text { nuclear localisation sequence } \\ \text { NR } & \text { N-terminal regulatory region } \\ \text { NTC } & \text { no template control } \\ \text { OSCC } & \text { oral squamous cell carcinoma } \\ \text { PCNA } & \text { proliferating cell nuclear antigen } \\ \text { PCR } & \text { polymerase chain reaction } \\ \text { PDB } & \text { protein databank } \\ \text { PH } & \text { plekstrin homology } \\ \text { PM } & \text { perfect match } \\ \text { PyMOL } & \text { Python-enhanced molecular graphics tool (software) } \\ \text { REMARK } & \text { reporting recommendations for tumour marker prognostic studies } \\ \text { RIN } & \text { RNA integrity number } \\ \text { RMA } & \text { robust multiarray average } \\ \text { RT } & \text { radiation therapy } \\ \text { RTK } & \text { receptor tyrosine kinase } \\ \text { S } & \text { Svedberg Unit, sedimentation } \\ \text { siRNA } & \text { small interfering RNA } \\ \text { SNP } & \text { single nucleotide polymorphism } \\ \text { SPF } & \text { s-phase fraction } \\ \text { SSCP } & \text { single-strand conformational polymorphism } \\ \text { TMA } & \text { tissue microarray } \\ \text { TNM } & \text { tumour, node, metastasis } \\ \text { TOP } & \text { 5'terminal oligopyrimidine tract } \\ \text { TOS } & \text { tor signalling motif } \\ \text { UICC } & \text { Union for International Cancer Control } \\ \text { ZR751 } & \text { breast cancer cell line } \\ & \end{array}$


1. Vogelstein B, Kinzler KW: The genetic basis of human cancer. McGraw-Hill companies 1998 1998, ISBN 0-07-067596-1.

2. Cancerfondsrapporten. 2012.

3. Fackenthal JD, Olopade OI: Breast cancer risk associated with BRCA1 and BRCA2 in diverse populations. Nat Rev Cancer 2007, 7(12):937-948.

4. Svensk förening för medicinsk genetik, SFMG. http://sfmgse/ 2013.

5. Lakhani SR, Ellis I, S.J S, Tan PH, van de Vijver MJ: WHO Classification of Tumours of the Breast, Fourth Edition. IARC Press 2012:13-59.

6. Svenska bröstcancergruppens nationella riktlinjer för behandling av bröstcancer. 2013.

7. Baselga J, Campone M, Piccart M, Burris HA, 3rd, Rugo HS, Sahmoud T, Noguchi S, Gnant $\mathrm{M}$, Pritchard KI, Lebrun F et al: Everolimus in postmenopausal hormone-receptorpositive advanced breast cancer. $N$ Engl J Med 2012, 366(6):520-529.

8. Simpson PT, Reis-Filho JS, Gale T, Lakhani SR: Molecular evolution of breast cancer. The Journal of pathology 2005, 205(2):248-254.

9. Goldhirsch A, Wood WC, Coates AS, Gelber RD, Thurlimann B, Senn HJ: Strategies for subtypes--dealing with the diversity of breast cancer: highlights of the St. Gallen International Expert Consensus on the Primary Therapy of Early Breast Cancer 2011. Annals of oncology : official journal of the European Society for Medical Oncology / ESMO 2011, 22(8):1736-1747.

10. Prat A, Cheang MC, Martin M, Parker JS, Carrasco E, Caballero R, Tyldesley S, Gelmon K, Bernard PS, Nielsen TO et al: Prognostic significance of progesterone receptor-positive tumor cells within immunohistochemically defined luminal A breast cancer. Journal of clinical oncology : official journal of the American Society of Clinical Oncology 2013, 31(2):203-209.

11. Lindström L, Howell S, Åström G, Wilking U, Lidbrink E, Armstron A, Karlsson E, Hatschek T, Bergh J: Contoversies in the management of metastatic breast cancer: biologic evaluation of breast cancer-should metastases be biopsied? ASCO, 20102010.

12. Musgrove EA, Sutherland RL: Biological determinants of endocrine resistance in breast cancer. Nat Rev Cancer 2009, 9(9):631-643.

13. Miller TW: Endocrine resistance. American Society of Clinical Oncology educational book / ASCO American Society of Clinical Oncology Meeting 2013, 2013:37-42.

14. Perou CM, Sorlie T, Eisen MB, van de Rijn M, Jeffrey SS, Rees CA, Pollack JR, Ross DT, Johnsen H, Akslen LA et al: Molecular portraits of human breast tumours. Nature 2000, 406(6797):747-752.

15. Polyak K: Breast cancer: origins and evolution. The Journal of clinical investigation 2007 , 117(11):3155-3163.

16. Sorlie T: Molecular portraits of breast cancer: tumour subtypes as distinct disease entities. European journal of cancer 2004, 40(18):2667-2675.

17. Patani N, Martin LA, Dowsett M: Biomarkers for the clinical management of breast cancer: international perspective. International journal of cancer Journal international du cancer 2013, 133(1):1-13.

18. Wood LD, Parsons DW, Jones S, Lin J, Sjoblom T, Leary RJ, Shen D, Boca SM, Barber T, Ptak $\mathrm{J}$ et al: The genomic landscapes of human breast and colorectal cancers. Science 2007, 318(5853):1108-1113.

19. Bergamaschi A, Kim YH, Wang P, Sorlie T, Hernandez-Boussard T, Lonning PE, Tibshirani R, Borresen-Dale AL, Pollack JR: Distinct patterns of DNA copy number alteration are associated with different clinicopathological features and gene-expression subtypes of breast cancer. Genes, chromosomes \& cancer 2006, 45(11):1033-1040. 
20. Hanahan D, Weinberg RA: The hallmarks of cancer. Cell 2000, 100(1):57-70.

21. Hanahan D, Weinberg RA: Hallmarks of cancer: the next generation. Cell 2011, 144(5):646-674.

22. Prat A, Perou CM: Mammary development meets cancer genomics. Nature medicine 2009, 15(8):842-844.

23. Lim E, Vaillant F, Wu D, Forrest NC, Pal B, Hart AH, Asselin-Labat ML, Gyorki DE, Ward $\mathrm{T}$, Partanen A et al: Aberrant luminal progenitors as the candidate target population for basal tumor development in BRCA1 mutation carriers. Nature medicine 2009, 15(8):907913.

24. Gjorevski N, Nelson CM: Integrated morphodynamic signalling of the mammary gland. Nat Rev Mol Cell Biol 2011, 12(9):581-593.

25. Shackleton M, Quintana E, Fearon ER, Morrison SJ: Heterogeneity in cancer: cancer stem cells versus clonal evolution. Cell 2009, 138(5):822-829.

26. Badve S, Nakshatri H: Breast-cancer stem cells-beyond semantics. The lancet oncology 2012, 13(1):e43-48.

27. Beatson GT: On the treatment of inoperable cases of carcinoma of the mamma: suggestions for a new method of treatment with illustrative cases. Lancet 1896, 2:104107.

28. Jensen EV, Jacobson HI: Basic guides to the mechanism of estrogen action. Recent Prog Horm Res 1962, 18:318-414.

29. Jensen EV, Block GE, Smith S, Kyser K, DeSombre ER: Estrogen receptors and breast cancer response to adrenalectomy. National Cancer Institute monograph 1971, 34:55-70.

30. Björnström L, Sjöberg M: Mechanisms of estrogen receptor signaling: convergence of genomic and nongenomic actions on target genes. Molecular endocrinology 2005, 19(4):833-842.

31. Obr AE, Edwards DP: The biology of progesterone receptor in the normal mammary gland and in breast cancer. Molecular and cellular endocrinology 2012, 357(1-2):4-17.

32. Brisken C: Progesterone signalling in breast cancer: a neglected hormone coming into the limelight. Nat Rev Cancer 2013, 13(6):385-396.

33. Prall OW, Rogan EM, Musgrove EA, Watts CK, Sutherland RL: c-Myc or cyclin D1 mimics estrogen effects on cyclin E-Cdk2 activation and cell cycle reentry. Mol Cell Biol 1998, 18(8):4499-4508.

34. Massarweh S, Schiff R: Resistance to endocrine therapy in breast cancer: exploiting estrogen receptor/growth factor signaling crosstalk. Endocrine-related cancer 2006, 13 Suppl 1:S15-24.

35. Aka JA, Mazumdar M, Lin SX: Reductive 17beta-hydroxysteroid dehydrogenases in the sulfatase pathway: critical in the cell proliferation of breast cancer. Molecular and cellular endocrinology 2009, 301(1-2):183-190.

36. Fresno Vara JA, Casado E, de Castro J, Cejas P, Belda-Iniesta C, Gonzalez-Baron M: PI3K/Akt signalling pathway and cancer. Cancer treatment reviews 2004, 30(2):193-204.

37. Osborne $\mathrm{C}$, Wilson $\mathrm{P}$, Tripathy $\mathrm{D}$ : Oncogenes and tumor suppressor genes in breast cancer: potential diagnostic and therapeutic applications. The oncologist 2004, 9(4):361377.

38. Warburg O: On respiratory impairment in cancer cells. Science 1956, 124(3215):269-270.

39. Warburg O: On the origin of cancer cells. Science 1956, 123(3191):309-314.

40. Hsu PP, Sabatini DM: Cancer cell metabolism: Warburg and beyond. Cell 2008, 134(5):703-707.

41. Koppenol WH, Bounds PL, Dang CV: Otto Warburg's contributions to current concepts of cancer metabolism. Nat Rev Cancer 2011, 11(5):325-337.

42. Tait SW, Green DR: Mitochondria and cell death: outer membrane permeabilization and beyond. Nat Rev Mol Cell Biol 2010, 11(9):621-632.

43. Bailey ST, Shin H, Westerling T, Liu XS, Brown M: Estrogen receptor prevents p53dependent apoptosis in breast cancer. Proc Natl Acad Sci U S A 2012, 109(44):1806018065 . 
44. Hayflick L: Mortality and immortality at the cellular level. A review. Biochemistry Biokhimiia 1997, 62(11):1180-1190.

45. Mocellin S, Pooley KA, Nitti D: Telomerase and the search for the end of cancer. Trends in molecular medicine 2013, 19(2):125-133.

46. Barcellos-Hoff MH, Medina D: New highlights on stroma-epithelial interactions in breast cancer. Breast Cancer Res 2005, 7(1):33-36.

47. Smith AL, Robin TP, Ford HL: Molecular pathways: targeting the TGF-beta pathway for cancer therapy. Clinical cancer research : an official journal of the American Association for Cancer Research 2012, 18(17):4514-4521.

48. Sasano H, Miki Y, Nagasaki S, Suzuki T: In situ estrogen production and its regulation in human breast carcinoma: from endocrinology to intracrinology. Pathology international 2009, 59(11):777-789.

49. Nelson LR, Bulun SE: Estrogen production and action. Journal of the American Academy of Dermatology 2001, 45(3 Suppl):S116-124.

50. Jiang X, Shapiro DJ: The immune system and inflammation in breast cancer. Molecular and cellular endocrinology 2013.

51. Sporn MB: The war on cancer. Lancet 1996, 347(9012):1377-1381.

52. Chui MH: Insights into cancer metastasis from a clinicopathologic perspective:

Epithelial-Mesenchymal Transition is not a necessary step. International journal of cancer Journal international du cancer 2013, 132(7):1487-1495.

53. Albertson DG: Gene amplification in cancer. Trends in genetics : TIG 2006, 22(8):447-455.

54. Myllykangas S, Bohling T, Knuutila S: Specificity, selection and significance of gene amplifications in cancer. Seminars in cancer biology 2007, 17(1):42-55.

55. Schwab M: Amplification of oncogenes in human cancer cells. BioEssays : news and reviews in molecular, cellular and developmental biology 1998, 20(6):473-479.

56. Hyman E, Kauraniemi P, Hautaniemi S, Wolf M, Mousses S, Rozenblum E, Ringner M, Sauter G, Monni O, Elkahloun A et al: Impact of DNA amplification on gene expression patterns in breast cancer. Cancer Res 2002, 62(21):6240-6245.

57. Pollack JR, Sorlie T, Perou CM, Rees CA, Jeffrey SS, Lonning PE, Tibshirani R, Botstein D, Borresen-Dale AL, Brown PO: Microarray analysis reveals a major direct role of DNA copy number alteration in the transcriptional program of human breast tumors. Proc Natl Acad Sci U S A 2002, 99(20):12963-12968.

58. Al-Kuraya K, Schraml P, Torhorst J, Tapia C, Zaharieva B, Novotny H, Spichtin H, Maurer $\mathrm{R}$, Mirlacher M, Kochli $\mathrm{O}$ et al: Prognostic relevance of gene amplifications and coamplifications in breast cancer. Cancer Res 2004, 64(23):8534-8540.

59. Cuny M, Kramar A, Courjal F, Johannsdottir V, Iacopetta B, Fontaine H, Grenier J, Culine S, Theillet $C$ : Relating genotype and phenotype in breast cancer: an analysis of the prognostic significance of amplification at eight different genes or loci and of p53 mutations. Cancer Res 2000, 60(4):1077-1083.

60. Couch FJ, Wang XY, Wu GJ, Qian J, Jenkins RB, James CD: Localization of PS6K to chromosomal region $17 \mathrm{q} 23$ and determination of its amplification in breast cancer. Cancer Res 1999, 59(7):1408-1411.

61. Bärlund M, Monni O, Kononen J, Cornelison R, Torhorst J, Sauter G, Kallioniemi O-P, Kallioniemi A: Multiple genes at 17q23 undergo amplification and overexpression in breast cancer. Cancer Res 2000, 60(19):5340-5344.

62. van der Hage JA, van den Broek LJ, Legrand C, Clahsen PC, Bosch CJ, Robanus-Maandag EC, van de Velde CJ, van de Vijver MJ: Overexpression of P70 S6 kinase protein is associated with increased risk of locoregional recurrence in node-negative premenopausal early breast cancer patients. Br J Cancer 2004, 90(8):1543-1550.

63. Noh WC, Kim YH, Kim MS, Koh JS, Kim HA, Moon NM, Paik NS: Activation of the mTOR signaling pathway in breast cancer and its correlation with the clinicopathologic variables. Breast Cancer Res Treat 2008, 110(3):477-483.

64. Myllykangas S, Knuutila S: Manifestation, mechanisms and mysteries of gene amplifications. Cancer letters 2006, 232(1):79-89. 
65. McClintock B: The Fusion of Broken Ends of Chromosomes Following Nuclear Fusion. Proc Natl Acad Sci U S A 1942, 28(11):458-463.

66. Myllykangas S, Himberg J, Bohling T, Nagy B, Hollmen J, Knuutila S: DNA copy number amplification profiling of human neoplasms. Oncogene 2006, 25(55):7324-7332.

67. Wahl GM, Padgett RA, Stark GR: Gene amplification causes overproduction of the first three enzymes of UMP synthesis in N-(phosphonacetyl)-L-aspartate-resistant hamster cells. J Biol Chem 1979, 254(17):8679-8689.

68. Dickson C, Fantl V, Gillett C, Brookes S, Bartek J, Smith R, Fisher C, Barnes D, Peters G: Amplification of chromosome band 11q13 and a role for cyclin D1 in human breast cancer. Cancer letters 1995, 90(1):43-50.

69. Tsujimoto Y, Yunis J, Onorato-Showe L, Erikson J, Nowell PC, Croce CM: Molecular cloning of the chromosomal breakpoint of B-cell lymphomas and leukemias with the t(11;14) chromosome translocation. Science 1984, 224(4656):1403-1406.

70. Arnold A, Kim HG, Gaz RD, Eddy RL, Fukushima Y, Byers MG, Shows TB, Kronenberg HM: Molecular cloning and chromosomal mapping of DNA rearranged with the parathyroid hormone gene in a parathyroid adenoma. The Journal of clinical investigation 1989, 83(6):2034-2040.

71. Schuuring E, Verhoeven E, Mooi WJ, Michalides RJ: Identification and cloning of two overexpressed genes, U21B31/PRAD1 and EMS1, within the amplified chromosome $11 q 13$ region in human carcinomas. Oncogene 1992, 7(2):355-361.

72. Wilkerson PM, Reis-Filho JS: The 11q13-q14 amplicon: clinicopathological correlations and potential drivers. Genes, chromosomes \& cancer 2013, 52(4):333-355.

73. Borg Å, Sigurdsson H, Clark GM, Fernö M, Fuqua SA, Olsson H, Killander D, McGurie WL: Association of INT2/HST1 coamplification in primary breast cancer with hormonedependent phenotype and poor prognosis. Br J Cancer 1991, 63(1):136-142.

74. Bostner J, Ahnström Waltersson M, Fornander T, Skoog L, Nordenskjöld B, Stål O: Amplification of CCND1 and PAK1 as predictors of recurrence and tamoxifen resistance in postmenopausal breast cancer. Oncogene 2007, 26(49):6997-7005.

75. Henry JA, Hennessy C, Levett DL, Lennard TW, Westley BR, May FE: int-2 amplification in breast cancer: association with decreased survival and relationship to amplification of c-erbB-2 and c-myc. International journal of cancer Journal international du cancer 1993, 53(5):774-780.

76. Karlseder J, Zeillinger R, Schneeberger C, Czerwenka K, Speiser P, Kubista E, Birnbaum D, Gaudray P, Theillet C: Patterns of DNA amplification at band q13 of chromosome 11 in human breast cancer. Genes, chromosomes \& cancer 1994, 9(1):42-48.

77. Letessier A, Sircoulomb F, Ginestier C, Cervera N, Monville F, Gelsi-Boyer V, Esterni B, Geneix J, Finetti $\mathrm{P}$, Zemmour $\mathrm{C}$ et al: Frequency, prognostic impact, and subtype association of 8p12, 8q24, 11q13, 12p13, 17q12, and $20 q 13$ amplifications in breast cancers. BMC cancer 2006, 6:245.

78. Schuuring E, Verhoeven E, van Tinteren H, Peterse JL, Nunnink B, Thunnissen FB, Devilee $\mathrm{P}$, Cornelisse CJ, van de Vijver MJ, Mooi WJ et al: Amplification of genes within the chromosome $11 \mathrm{q} 13$ region is indicative of poor prognosis in patients with operable breast cancer. Cancer Res 1992, 52(19):5229-5234.

79. Alle KM, Henshall SM, Field AS, Sutherland RL: Cyclin D1 protein is overexpressed in hyperplasia and intraductal carcinoma of the breast. Clinical cancer research : an official journal of the American Association for Cancer Research 1998, 4(4):847-854.

80. Simpson JF, Quan DE, O'Malley F, Odom-Maryon T, Clarke PE: Amplification of CCND1 and expression of its protein product, cyclin D1, in ductal carcinoma in situ of the breast. The American journal of pathology 1997, 151(1):161-168.

81. Weinstat-Saslow D, Merino MJ, Manrow RE, Lawrence JA, Bluth RF, Wittenbel KD, Simpson JF, Page DL, Steeg PS: Overexpression of cyclin D mRNA distinguishes invasive and in situ breast carcinomas from non-malignant lesions. Nature medicine 1995, 1(12):1257-1260. 
82. Schuuring E: The involvement of the chromosome 11q13 region in human malignancies: cyclin D1 and EMS1 are two new candidate oncogenes--a review. Gene 1995, 159(1):8396.

83. Brown LA, Kalloger SE, Miller MA, Shih Ie M, McKinney SE, Santos JL, Swenerton K, Spellman PT, Gray J, Gilks CB et al: Amplification of 11q13 in ovarian carcinoma. Genes, chromosomes \& cancer 2008, 47(6):481-489.

84. Ormandy CJ, Musgrove EA, Hui R, Daly RJ, Sutherland RL: Cyclin D1, EMS1 and $11 q 13$ amplification in breast cancer. Breast Cancer Res Treat 2003, 78(3):323-335.

85. Gibcus JH, Kok K, Menkema L, Hermsen MA, Mastik M, Kluin PM, van der Wal JE, Schuuring E: High-resolution mapping identifies a commonly amplified 11q13.3 region containing multiple genes flanked by segmental duplications. Hum Genet 2007, 121(2):187-201.

86. Elsheikh S, Green AR, Aleskandarany MA, Grainge M, Paish CE, Lambros MB, Reis-Filho JS, Ellis IO: CCND1 amplification and cyclin D1 expression in breast cancer and their relation with proteomic subgroups and patient outcome. Breast Cancer Res Treat 2008, 109(2):325-335.

87. Courjal F, Cuny M, Simony-Lafontaine J, Louason G, Speiser P, Zeillinger R, Rodriguez C, Theillet C: Mapping of DNA amplifications at 15 chromosomal localizations in 1875 breast tumors: definition of phenotypic groups. Cancer Res 1997, 57(19):4360-4367.

88. Reis-Filho JS, Savage K, Lambros MB, James M, Steele D, Jones RL, Dowsett M: Cyclin D1 protein overexpression and CCND1 amplification in breast carcinomas: an immunohistochemical and chromogenic in situ hybridisation analysis. Modern pathology : an official journal of the United States and Canadian Academy of Pathology, Inc 2006, 19(7):999-1009.

89. Dib A, Adelaide J, Chaffanet M, Imbert A, Le Paslier D, Jacquemier J, Gaudray P, Theillet C, Birnbaum D, Pebusque MJ: Characterization of the region of the short arm of chromosome 8 amplified in breast carcinoma. Oncogene 1995, 10(5):995-1001.

90. Garcia MJ, Pole JC, Chin SF, Teschendorff A, Naderi A, Ozdag H, Vias M, Kranjac T, Subkhankulova T, Paish $\mathrm{C}$ et al: A 1 Mb minimal amplicon at 8p11-12 in breast cancer identifies new candidate oncogenes. Oncogene 2005, 24(33):5235-5245.

91. Paterson AL, Pole JC, Blood KA, Garcia MJ, Cooke SL, Teschendorff AE, Wang Y, Chin $\mathrm{SF}$, Ylstra B, Caldas $\mathrm{C}$ et al: Co-amplification of 8p12 and 11q13 in breast cancers is not the result of a single genomic event. Genes, chromosomes \& cancer 2007, 46(5):427-439.

92. Guertin DA, Sabatini DM: Defining the role of mTOR in cancer. Cancer Cell 2007, 12(1):9-22.

93. Miller TW, Balko JM, Arteaga CL: Phosphatidylinositol 3-kinase and antiestrogen resistance in breast cancer. Journal of clinical oncology : official journal of the American Society of Clinical Oncology 2011, 29(33):4452-4461.

94. Guevara-Aguirre J, Balasubramanian P, Guevara-Aguirre M, Wei M, Madia F, Cheng CW, Hwang D, Martin-Montalvo A, Saavedra J, Ingles S et al: Growth hormone receptor deficiency is associated with a major reduction in pro-aging signaling, cancer, and diabetes in humans. Sci Transl Med 2011, 3(70):70ra13.

95. Manning G, Whyte DB, Martinez R, Hunter T, Sudarsanam S: The protein kinase complement of the human genome. Science 2002, 298(5600):1912-1934.

96. Taylor SS, Kornev AP: Protein kinases: evolution of dynamic regulatory proteins. Trends Biochem Sci 2011, 36(2):65-77.

97. Gschwind A, Fischer OM, Ullrich A: The discovery of receptor tyrosine kinases: targets for cancer therapy. Nat Rev Cancer 2004, 4(5):361-370.

98. Lemmon MA, Schlessinger J: Cell signaling by receptor tyrosine kinases. Cell 2010, 141(7):1117-1134.

99. Whitman M, Kaplan DR, Schaffhausen B, Cantley L, Roberts TM: Association of phosphatidylinositol kinase activity with polyoma middle-T competent for transformation. Nature 1985, 315(6016):239-242. 
100. Whitman M, Downes CP, Keeler M, Keller T, Cantley L: Type I phosphatidylinositol kinase makes a novel inositol phospholipid, phosphatidylinositol-3-phosphate. Nature 1988, 332(6165):644-646.

101. Volinia S, Hiles I, Ormondroyd E, Nizetic D, Antonacci R, Rocchi M, Waterfield MD: Molecular cloning, cDNA sequence, and chromosomal localization of the human phosphatidylinositol 3-kinase p110 alpha (PIK3CA) gene. Genomics 1994, 24(3):472-477.

102. Cidado J, Park BH: Targeting the PI3K/Akt/mTOR pathway for breast cancer therapy. Journal of mammary gland biology and neoplasia 2012, 17(3-4):205-216.

103. Stambolic V, Woodgett JR: Functional distinctions of protein kinase B/Akt isoforms defined by their influence on cell migration. Trends in cell biology 2006, 16(9):461-466.

104. Manning BD, Cantley LC: AKT/PKB signaling: navigating downstream. Cell 2007, 129(7):1261-1274.

105. Heitman J, Movva NR, Hall MN: Targets for cell cycle arrest by the immunosuppressant rapamycin in yeast. Science 1991, 253(5022):905-909.

106. Yang H, Rudge DG, Koos JD, Vaidialingam B, Yang HJ, Pavletich NP: mTOR kinase structure, mechanism and regulation. Nature 2013, 497(7448):217-223.

107. Kim DH, Sarbassov DD, Ali SM, King JE, Latek RR, Erdjument-Bromage H, Tempst P, Sabatini DM: mTOR interacts with raptor to form a nutrient-sensitive complex that signals to the cell growth machinery. Cell 2002, 110(2):163-175.

108. Loewith R, Jacinto E, Wullschleger S, Lorberg A, Crespo JL, Bonenfant D, Oppliger W, Jenoe $\mathrm{P}$, Hall MN: Two TOR complexes, only one of which is rapamycin sensitive, have distinct roles in cell growth control. Mol Cell 2002, 10(3):457-468.

109. Dunlop EA, Tee AR: Mammalian target of rapamycin complex 1: signalling inputs, substrates and feedback mechanisms. Cellular signalling 2009, 21(6):827-835.

110. Chiang GG, Abraham RT: Phosphorylation of mammalian target of rapamycin (mTOR) at Ser-2448 is mediated by p70S6 kinase. $J$ Biol Chem 2005, 280(27):25485-25490.

111. Holz MK, Blenis J: Identification of S6 kinase 1 as a novel mammalian target of rapamycin (mTOR)-phosphorylating kinase. J Biol Chem 2005, 280(28):26089-26093.

112. Inoki K, Ouyang H, Zhu T, Lindvall C, Wang Y, Zhang X, Yang Q, Bennett C, Harada Y, Stankunas $\mathrm{K}$ et al: TSC2 integrates Wnt and energy signals via a coordinated phosphorylation by AMPK and GSK3 to regulate cell growth. Cell 2006, 126(5):955-968.

113. Ma XM, Blenis J: Molecular mechanisms of mTOR-mediated translational control. Nat Rev Mol Cell Biol 2009, 10(5):307-318.

114. Sarbassov DD, Guertin DA, Ali SM, Sabatini DM: Phosphorylation and regulation of Akt/PKB by the rictor-mTOR complex. Science 2005, 307(5712):1098-1101.

115. Fenton TR, Gout IT: Functions and regulation of the 70kDa ribosomal S6 kinases. Int $J$ Biochem Cell Biol 2010, 43(1):47-59.

116. Ismail HM, Myronova O, Tsuchiya Y, Niewiarowski A, Tsaneva I, Gout I: Identification of the general transcription factor Yin Yang 1 as a novel and specific binding partner for S6 kinase 2. Cellular signalling 2013, 25(5):1054-1063.

117. Pardo OE, Arcaro A, Salerno G, Tetley TD, Valovka T, Gout I, Seckl MJ: Novel cross talk between MEK and S6K2 in FGF-2 induced proliferation of SCLC cells. Oncogene 2001, 20(52):7658-7667.

118. Pardo OE, Seckl MJ: S6K2: The Neglected S6 Kinase Family Member. Frontiers in oncology 2013, 3:191.

119. Pardo OE, Wellbrock C, Khanzada UK, Aubert M, Arozarena I, Davidson S, Bowen F, Parker PJ, Filonenko VV, Gout IT et al: FGF-2 protects small cell lung cancer cells from apoptosis through a complex involving PKCepsilon, B-Raf and S6K2. EMBO J 2006, 25(13):3078-3088.

120. Goh ET, Pardo OE, Michael N, Niewiarowski A, Totty N, Volkova D, Tsaneva IR, Seckl MJ, Gout I: Involvement of heterogeneous ribonucleoprotein $\mathrm{F}$ in the regulation of cell proliferation via the mammalian target of rapamycin/S6 kinase 2 pathway. J Biol Chem 2010, 285(22):17065-17076. 
121. Magnuson B, Ekim B, Fingar DC: Regulation and function of ribosomal protein S6 kinase (S6K) within mTOR signalling networks. Biochem J 2012, 441(1):1-21.

122. Silvera D, Formenti SC, Schneider RJ: Translational control in cancer. Nat Rev Cancer 2010, 10(4):254-266.

123. Lempiainen H, Shore D: Growth control and ribosome biogenesis. Current opinion in cell biology 2009, 21(6):855-863.

124. Ma XM, Yoon SO, Richardson CJ, Julich K, Blenis J: SKAR links pre-mRNA splicing to mTOR/S6K1-mediated enhanced translation efficiency of spliced mRNAs. Cell 2008, 133(2):303-313.

125. Ruvinsky I, Meyuhas O: Ribosomal protein S6 phosphorylation: from protein synthesis to cell size. Trends Biochem Sci 2006, 31(6):342-348.

126. Jeno P, Ballou LM, Novak-Hofer I, Thomas G: Identification and characterization of a mitogen-activated S6 kinase. Proc Natl Acad Sci U S A 1988, 85(2):406-410.

127. Nemenoff RA, Price DJ, Mendelsohn MJ, Carter EA, Avruch J: An S6 kinase activated during liver regeneration is related to the insulin-stimulated $\mathrm{S6}$ kinase in $\mathrm{H4}$ hepatoma cells. J Biol Chem 1988, 263(36):19455-19460.

128. Harmann B, Kilimann MW: cDNA encoding a 59 kDa homolog of ribosomal protein S6 kinase from rabbit liver. FEBS Lett 1990, 273(1-2):248-252.

129. Banerjee P, Ahmad MF, Grove JR, Kozlosky C, Price DJ, Avruch J: Molecular structure of a major insulin/mitogen-activated 70-kDa S6 protein kinase. Proc Natl Acad Sci U S A 1990, 87(21):8550-8554.

130. Kozma SC, Ferrari S, Bassand P, Siegmann M, Totty N, Thomas G: Cloning of the mitogenactivated $S 6$ kinase from rat liver reveals an enzyme of the second messenger subfamily. Proc Natl Acad Sci U S A 1990, 87(19):7365-7369.

131. Grove JR, Banerjee P, Balasubramanyam A, Coffer PJ, Price DJ, Avruch J, Woodgett JR: Cloning and expression of two human p70 S6 kinase polypeptides differing only at their amino termini. Mol Cell Biol 1991, 11(11):5541-5550.

132. Gout I, Minami T, Hara K, Tsujishita Y, Filonenko V, Waterfield MD, Yonezawa K: Molecular cloning and characterization of a novel p70 S6 kinase, p70 S6 kinase beta containing a proline-rich region. $J$ Biol Chem 1998, 273(46):30061-30064.

133. Koh H, Jee K, Lee B, Kim J, Kim D, Yun YH, Kim JW, Choi HS, Chung J: Cloning and characterization of a nuclear S6 kinase, S6 kinase-related kinase (SRK); a novel nuclear target of Akt. Oncogene 1999, 18(36):5115-5119.

134. Lee-Fruman KK, Kuo CJ, Lippincott J, Terada N, Blenis J: Characterization of S6K2, a novel kinase homologous to S6K1. Oncogene 1999, 18(36):5108-5114.

135. Saitoh M, ten Dijke P, Miyazono K, Ichijo H: Cloning and characterization of p70(S6K beta) defines a novel family of $\mathbf{p 7 0} \mathrm{S6}$ kinases. Biochemical and biophysical research communications 1998, 253(2):470-476.

136. Karni R, de Stanchina E, Lowe SW, Sinha R, Mu D, Krainer AR: The gene encoding the splicing factor SF2/ASF is a proto-oncogene. Nat Struct Mol Biol 2007, 14(3):185-193.

137. Burnett PE, Blackshaw S, Lai MM, Qureshi IA, Burnett AF, Sabatini DM, Snyder SH: Neurabin is a synaptic protein linking p70 $\mathbf{S 6}$ kinase and the neuronal cytoskeleton. Proc Natl Acad Sci U S A 1998, 95(14):8351-8356.

138. Zhou D, Ye JJ, Li Y, Lui K, Chen S: The molecular basis of the interaction between the proline-rich SH3-binding motif of PNRC and estrogen receptor alpha. Nucleic Acids Res 2006, 34(20):5974-5986.

139. Jastrzebski K, Hannan KM, Tchoubrieva EB, Hannan RD, Pearson RB: Coordinate regulation of ribosome biogenesis and function by the ribosomal protein S6 kinase, a key mediator of mTOR function. Growth Factors 2007, 25(4):209-226.

140. Shima H, Pende M, Chen Y, Fumagalli S, Thomas G, Kozma SC: Disruption of the p70(s6k)/p85(s6k) gene reveals a small mouse phenotype and a new functional S6 kinase. Embo J 1998, 17(22):6649-6659.

141. Nardella C, Lunardi A, Fedele G, Clohessy JG, Alimonti A, Kozma SC, Thomas G, Loda M, Pandolfi PP: Differential expression of S6K2 dictates tissue-specific requirement for 
S6K1 in mediating aberrant mTORC1 signaling and tumorigenesis. Cancer Res 2011, 71(10):3669-3675.

142. Sunami T, Byrne N, Diehl RE, Funabashi K, Hall DL, Ikuta M, Patel SB, Shipman JM, Smith $\mathrm{RF}$, Takahashi I et al: Structural basis of human p70 ribosomal $\mathbf{S 6}$ kinase-1 regulation by activation loop phosphorylation. $J$ Biol Chem 2010, 285(7):4587-4594.

143. Wang J, Zhong C, Wang F, Qu F, Ding J: Crystal structures of S6K1 provide insights into the regulation mechanism of S6K1 by the hydrophobic motif. Biochem J 2013, 454(1):3947.

144. Pearce LR, Alton GR, Richter DT, Kath JC, Lingardo L, Chapman J, Hwang C, Alessi DR: Characterization of PF-4708671, a novel and highly specific inhibitor of p70 ribosomal S6 kinase (S6K1). Biochem J 2010, 431(2):245-255.

145. Chung J, Kuo CJ, Crabtree GR, Blenis J: Rapamycin-FKBP specifically blocks growthdependent activation of and signaling by the $70 \mathrm{kd}$ S6 protein kinases. Cell 1992, 69(7):1227-1236.

146. Kuo CJ, Chung J, Fiorentino DF, Flanagan WM, Blenis J, Crabtree GR: Rapamycin selectively inhibits interleukin-2 activation of p70 S6 kinase. Nature 1992, 358(6381):7073.

147. Price DJ, Grove JR, Calvo V, Avruch J, Bierer BE: Rapamycin-induced inhibition of the 70-kilodalton S6 protein kinase. Science 1992, 257(5072):973-977.

148. Isotani S, Hara K, Tokunaga C, Inoue H, Avruch J, Yonezawa K: Immunopurified mammalian target of rapamycin phosphorylates and activates p70 S6 kinase alpha in vitro. J Biol Chem 1999, 274(48):34493-34498.

149. Phin S, Kupferwasser D, Lam J, Lee-Fruman KK: Mutational analysis of ribosomal S6 kinase 2 shows differential regulation of its kinase activity from that of ribosomal S6 kinase 1. Biochem J 2003, 373(Pt 2):583-591.

150. Panasyuk G, Nemazanyy I, Zhyvoloup A, Bretner M, Litchfield DW, Filonenko V, Gout IT: Nuclear export of S6K1 II is regulated by protein kinase CK2 phosphorylation at Ser17. J Biol Chem 2006, 281(42):31188-31201.

151. Rosner M, Schipany K, Hengstschlager M: p70 S6K1 nuclear localization depends on its mTOR-mediated phosphorylation at T389, but not on its kinase activity towards S6. Amino Acids 2011.

152. Panasyuk G, Nemazanyy I, Filonenko V, Gout I: Ribosomal protein S6 kinase 1 interacts with and is ubiquitinated by ubiquitin ligase ROC1. Biochem Biophys Res Commun 2008, 369(2):339-343.

153. Fenton TR, Gwalter J, Cramer R, Gout IT: S6K1 is acetylated at lysine 516 in response to growth factor stimulation. Biochem Biophys Res Commun 2010, 398(3):400-405.

154. Gwalter J, Wang ML, Gout I: The ubiquitination of ribosomal S6 kinases is independent from the mitogen-induced phosphorylation/activation of the kinase. Int J Biochem Cell Biol 2009, 41(4):828-833.

155. Rychlik W, Domier LL, Gardner PR, Hellmann GM, Rhoads RE: Amino acid sequence of the mRNA cap-binding protein from human tissues. Proc Natl Acad Sci U S A 1987, 84(4):945-949.

156. Pause A, Belsham GJ, Gingras AC, Donze O, Lin TA, Lawrence JC, Jr., Sonenberg N: Insulin-dependent stimulation of protein synthesis by phosphorylation of a regulator of 5'-cap function. Nature 1994, 371(6500):762-767.

157. Tsukiyama-Kohara K, Vidal SM, Gingras AC, Glover TW, Hanash SM, Heng H, Sonenberg $\mathrm{N}$ : Tissue distribution, genomic structure, and chromosome mapping of mouse and human eukaryotic initiation factor 4E-binding proteins 1 and 2. Genomics 1996, 38(3):353-363.

158. Poulin F, Gingras AC, Olsen H, Chevalier S, Sonenberg N: 4E-BP3, a new member of the eukaryotic initiation factor 4E-binding protein family. J Biol Chem 1998, 273(22):1400214007.

159. Poulin F, Brueschke A, Sonenberg N: Gene fusion and overlapping reading frames in the mammalian genes for 4E-BP3 and MASK. J Biol Chem 2003, 278(52):52290-52297. 
160. Sikalidis AK, Mazor KM, Kang M, Liu H, Stipanuk MH: Total 4EBP1 Is Elevated in Liver of Rats in Response to Low Sulfur Amino Acid Intake. Journal of amino acids 2013, 2013:864757.

161. Wang X, Li W, Williams M, Terada N, Alessi DR, Proud CG: Regulation of elongation factor 2 kinase by p90(RSK1) and p70 S6 kinase. Embo $J$ 2001, 20(16):4370-4379.

162. Carmo CR, Lyons-Lewis J, Seckl MJ, Costa-Pereira AP: A novel requirement for Janus kinases as mediators of drug resistance induced by fibroblast growth factor-2 in human cancer cells. PLoS One 2011, 6(5):e19861.

163. Pende M, Um SH, Mieulet V, Sticker M, Goss VL, Mestan J, Mueller M, Fumagalli S, Kozma SC, Thomas G: S6K1(-/-)/S6K2(-/-) mice exhibit perinatal lethality and rapamycin-sensitive 5'-terminal oligopyrimidine mRNA translation and reveal a mitogen-activated protein kinase-dependent S6 kinase pathway. Mol Cell Biol 2004, 24(8):3112-3124.

164. Ruvinsky I, Sharon N, Lerer T, Cohen H, Stolovich-Rain M, Nir T, Dor Y, Zisman P, Meyuhas O: Ribosomal protein $\mathrm{S} 6$ phosphorylation is a determinant of cell size and glucose homeostasis. Genes Dev 2005, 19(18):2199-2211.

165. Yamnik RL, Digilova A, Davis DC, Brodt ZN, Murphy CJ, Holz MK: S6 kinase 1 regulates estrogen receptor alpha in control of breast cancer cell proliferation. J Biol Chem 2009, 284(10):6361-6369.

166. Jastrzebski K, Hannan KM, House CM, Hung SS, Pearson RB, Hannan RD: A phosphoproteomic screen identifies novel S6K1 and mTORC1 substrates revealing additional complexity in the signaling network regulating cell growth. Cell Signal, 23(8):1338-1347.

167. Han SP, Tang YH, Smith R: Functional diversity of the hnRNPs: past, present and perspectives. Biochem J 2010, 430(3):379-392.

168. Boyer D, Quintanilla R, Lee-Fruman KK: Regulation of catalytic activity of S6 kinase 2 during cell cycle. Molecular and cellular biochemistry 2008, 307(1-2):59-64.

169. Rosner M, Hengstschlager M: Nucleocytoplasmic localization of p70 S6K1, but not of its isoforms p85 and p31, is regulated by TSC2/mTOR. Oncogene 2011.

170. Rossi R, Pester JM, McDowell M, Soza S, Montecucco A, Lee-Fruman KK: Identification of S6K2 as a centrosome-located kinase. FEBS letters 2007, 581(21):4058-4064.

171. Zhu J, Blenis J, Yuan J: Activation of PI3K/Akt and MAPK pathways regulates Mycmediated transcription by phosphorylating and promoting the degradation of Mad1. Proc Natl Acad Sci U S A 2008, 105(18):6584-6589.

172. Ben-Sahra I, Howell JJ, Asara JM, Manning BD: Stimulation of de novo pyrimidine synthesis by growth signaling through mTOR and S6K1. Science 2013, 339(6125):13231328.

173. Robitaille AM, Christen S, Shimobayashi M, Cornu M, Fava LL, Moes S, PrescianottoBaschong C, Sauer U, Jenoe P, Hall MN: Quantitative phosphoproteomics reveal mTORC1 activates de novo pyrimidine synthesis. Science 2013, 339(6125):1320-1323.

174. Montagne J, Stewart MJ, Stocker H, Hafen E, Kozma SC, Thomas G: Drosophila S6 kinase: a regulator of cell size. Science 1999, 285(5436):2126-2129.

175. Harada H, Andersen JS, Mann M, Terada N, Korsmeyer SJ: p70S6 kinase signals cell survival as well as growth, inactivating the pro-apoptotic molecule BAD. Proc Natl Acad Sci U S A 2001, 98(17):9666-9670.

176. Pende M, Kozma SC, Jaquet M, Oorschot V, Burcelin R, Le Marchand-Brustel Y, Klumperman J, Thorens B, Thomas G: Hypoinsulinaemia, glucose intolerance and diminished beta-cell size in S6K1-deficient mice. Nature 2000, 408(6815):994-997.

177. Um SH, Frigerio F, Watanabe M, Picard F, Joaquin M, Sticker M, Fumagalli S, Allegrini PR, Kozma SC, Auwerx J et al: Absence of S6K1 protects against age- and diet-induced obesity while enhancing insulin sensitivity. Nature 2004, 431(7005):200-205.

178. Miller TW, Rexer BN, Garrett JT, Arteaga CL: Mutations in the phosphatidylinositol 3kinase pathway: role in tumor progression and therapeutic implications in breast cancer. Breast Cancer Res 2011, 13(6):224. 
179. Perez-Tenorio G, Alkhori L, Olsson B, Waltersson MA, Nordenskjöld B, Rutqvist LE, Skoog L, Stål O: PIK3CA mutations and PTEN loss correlate with similar prognostic factors and are not mutually exclusive in breast cancer. Clinical cancer research : an official journal of the American Association for Cancer Research 2007, 13(12):3577-3584.

180. Kim EK, Kim HA, Koh JS, Kim MS, Kim KI, Lee JI, Moon NM, Ko E, Noh WC:

Phosphorylated S6K1 is a possible marker for endocrine therapy resistance in hormone receptor-positive breast cancer. Breast Cancer Res Treat 2011, 126(1):93-99.

181. Lyzogubov V, Khozhaenko Y, Usenko V, Antonjuk S, Ovcharenko G, Tikhonkova I, Filonenko V: Immunohistochemical analysis of Ki-67, PCNA and S6K1/2 expression in human breast cancer. Exp Oncol 2005, 27(2):141-144.

182. Ip CK, Cheung AN, Ngan HY, Wong AS: p70 S6 kinase in the control of actin cytoskeleton dynamics and directed migration of ovarian cancer cells. Oncogene 2011, 30(21):2420-2432.

183. Pon YL, Zhou HY, Cheung AN, Ngan HY, Wong AS: p70 S6 kinase promotes epithelial to mesenchymal transition through snail induction in ovarian cancer cells. Cancer Res 2008, 68(16):6524-6532.

184. Bernard-Pierrot I, Gruel N, Stransky N, Vincent-Salomon A, Reyal F, Raynal V, Vallot C, Pierron G, Radvanyi F, Delattre O: Characterization of the recurrent 8p11-12 amplicon identifies PPAPDC1B, a phosphatase protein, as a new therapeutic target in breast cancer. Cancer Res 2008, 68(17):7165-7175.

185. Ray ME, Yang ZQ, Albertson D, Kleer CG, Washburn JG, Macoska JA, Ethier SP: Genomic and expression analysis of the 8p11-12 amplicon in human breast cancer cell lines. Cancer Res 2004, 64(1):40-47.

186. Reyal F, Stransky N, Bernard-Pierrot I, Vincent-Salomon A, de Rycke Y, Elvin P, Cassidy A, Graham A, Spraggon C, Desille $Y$ et al: Visualizing chromosomes as transcriptome correlation maps: evidence of chromosomal domains containing co-expressed genes--a study of 130 invasive ductal breast carcinomas. Cancer Res 2005, 65(4):1376-1383.

187. Yang ZQ, Streicher KL, Ray ME, Abrams J, Ethier SP: Multiple interacting oncogenes on the 8p11-p12 amplicon in human breast cancer. Cancer Res 2006, 66(24):11632-11643.

188. Balakumaran BS, Porrello A, Hsu DS, Glover W, Foye A, Leung JY, Sullivan BA, Hahn WC, Loda M, Febbo PG: MYC activity mitigates response to rapamycin in prostate cancer through eukaryotic initiation factor $4 \mathrm{E}$-binding protein 1-mediated inhibition of autophagy. Cancer Res 2009, 69(19):7803-7810.

189. Castellvi J, Garcia A, Rojo F, Ruiz-Marcellan C, Gil A, Baselga J, Ramon y Cajal S: Phosphorylated 4E binding protein 1: a hallmark of cell signaling that correlates with survival in ovarian cancer. Cancer 2006, 107(8):1801-1811.

190. Rojo F, Najera L, Lirola J, Jimenez J, Guzman M, Sabadell MD, Baselga J, Ramon y Cajal S: 4E-binding protein 1 , a cell signaling hallmark in breast cancer that correlates with pathologic grade and prognosis. Clinical cancer research : an official journal of the American Association for Cancer Research 2007, 13(1):81-89.

191. Braunstein S, Karpisheva K, Pola C, Goldberg J, Hochman T, Yee H, Cangiarella J, Arju R, Formenti SC, Schneider RJ: A hypoxia-controlled cap-dependent to cap-independent translation switch in breast cancer. Mol Cell 2007, 28(3):501-512.

192. Wang L, Rhodes CJ, Lawrence JC, Jr.: Activation of mammalian target of rapamycin (mTOR) by insulin is associated with stimulation of $4 \mathrm{EBP1}$ binding to dimeric $\mathrm{mTOR}$ complex 1. J Biol Chem 2006, 281(34):24293-24303.

193. Rong L, Livingstone M, Sukarieh R, Petroulakis E, Gingras AC, Crosby K, Smith B, Polakiewicz RD, Pelletier J, Ferraiuolo MA et al: Control of eIF4E cellular localization by eIF4E-binding proteins, 4E-BPs. Rna 2008, 14(7):1318-1327.

194. Larsson SC, Mantzoros CS, Wolk A: Diabetes mellitus and risk of breast cancer: a metaanalysis. International journal of cancer Journal international du cancer 2007, 121(4):856862.

195. Peairs KS, Barone BB, Snyder CF, Yeh HC, Stein KB, Derr RL, Brancati FL, Wolff AC: Diabetes mellitus and breast cancer outcomes: a systematic review and meta-analysis. 
Journal of clinical oncology : official journal of the American Society of Clinical Oncology 2011, 29(1):40-46.

196. Potter DA, Yee D, Guo Z, Rodriguez M: Should diabetic women with breast cancer have their own intervention studies? Endocrine-related cancer 2012, 19(1):C13-17.

197. Olokoba AB, Obateru OA, Olokoba LB: Type 2 diabetes mellitus: a review of current trends. Oman medical journal 2012, 27(4):269-273.

198. Puri S, Hebrok M: Diabetic beta Cells: To Be or Not To Be? Cell 2012, 150(6):1103-1104

199. Talchai C, Xuan S, Lin HV, Sussel L, Accili D: Pancreatic beta cell dedifferentiation as a mechanism of diabetic beta cell failure. Cell 2012, 150(6):1223-1234.

200. Stattin P, Bjor O, Ferrari P, Lukanova A, Lenner P, Lindahl B, Hallmans G, Kaaks R: Prospective study of hyperglycemia and cancer risk. Diabetes care 2007, 30(3):561-567.

201. Villarreal-Garza C, Shaw-Dulin R, Lara-Medina F, Bacon L, Rivera D, Urzua L, Aguila C, Ramirez-Morales R, Santamaria J, Bargallo E et al: Impact of diabetes and hyperglycemia on survival in advanced breast cancer patients. Experimental diabetes research 2012, 2012:732027.

202. Tian J, Berton TR, Shirley SH, Lambertz I, Gimenez-Conti IB, DiGiovanni J, Korach KS, Conti CJ, Fuchs-Young R: Developmental stage determines estrogen receptor alpha expression and non-genomic mechanisms that control IGF-1 signaling and mammary proliferation in mice. The Journal of clinical investigation 2012, 122(1):192-204.

203. Viollet B, Guigas B, Sanz Garcia N, Leclerc J, Foretz M, Andreelli F: Cellular and molecular mechanisms of metformin: an overview. Clinical science 2012, 122(6):253-270.

204. Chlebowski RT, McTiernan A, Wactawski-Wende J, Manson JE, Aragaki AK, Rohan T, Ipp E, Kaklamani VG, Vitolins M, Wallace R et al: Diabetes, metformin, and breast cancer in postmenopausal women. Journal of clinical oncology : official journal of the American Society of Clinical Oncology 2012, 30(23):2844-2852.

205. Monami M, Colombi C, Balzi D, Dicembrini I, Giannini S, Melani C, Vitale V, Romano D, Barchielli A, Marchionni $\mathrm{N}$ et al: Metformin and cancer occurrence in insulin-treated type 2 diabetic patients. Diabetes care 2011, 34(1):129-131.

206. Bowker SL, Yasui Y, Veugelers P, Johnson JA: Glucose-lowering agents and cancer mortality rates in type 2 diabetes: assessing effects of time-varying exposure. Diabetologia 2010, 53(8):1631-1637.

207. Jalving M, Gietema JA, Lefrandt JD, de Jong S, Reyners AK, Gans RO, de Vries EG: Metformin: taking away the candy for cancer? European journal of cancer 2010, 46(13):2369-2380.

208. Dann SG, Selvaraj A, Thomas G: mTOR Complex1-S6K1 signaling: at the crossroads of obesity, diabetes and cancer. Trends in molecular medicine 2007, 13(6):252-259.

209. Campbell RA, Bhat-Nakshatri P, Patel NM, Constantinidou D, Ali S, Nakshatri H: Phosphatidylinositol 3-kinase/AKT-mediated activation of estrogen receptor alpha: a new model for anti-estrogen resistance. $J$ Biol Chem 2001, 276(13):9817-9824.

210. Kato S, Endoh H, Masuhiro Y, Kitamoto T, Uchiyama S, Sasaki H, Masushige S, Gotoh Y, Nishida E, Kawashima $\mathrm{H}$ et al: Activation of the estrogen receptor through

phosphorylation by mitogen-activated protein kinase. Science 1995, 270(5241):14911494.

211. Vartanian R, Masri J, Martin J, Cloninger C, Holmes B, Artinian N, Funk A, Ruegg T, Gera J: AP-1 regulates cyclin D1 and c-MYC transcription in an AKT-dependent manner in response to mTOR inhibition: role of AIP4/Itch-mediated JUNB degradation. Molecular cancer research: $M C R$ 2011, 9(1):115-130.

212. Bostner J, Karlsson E, Pandiyan MJ, Westman H, Skoog L, Fornander T, Nordenskjöld B, Stål O: Activation of Akt, mTOR, and the estrogen receptor as a signature to predict tamoxifen treatment benefit. Breast Cancer Res Treat 2013, 137(2):397-406.

213. Fox EM, Miller TW, Balko JM, Kuba MG, Sanchez V, Smith RA, Liu S, Gonzalez-Angulo AM, Mills GB, Ye F et al: A kinome-wide screen identifies the insulin/IGF-I receptor pathway as a mechanism of escape from hormone dependence in breast cancer. Cancer Res 2011, 71(21):6773-6784. 
214. Miller TW, Hennessy BT, Gonzalez-Angulo AM, Fox EM, Mills GB, Chen H, Higham C, Garcia-Echeverria C, Shyr Y, Arteaga CL: Hyperactivation of phosphatidylinositol-3 kinase promotes escape from hormone dependence in estrogen receptor-positive human breast cancer. The Journal of clinical investigation 2010, 120(7):2406-2413.

215. Perez-Tenorio G, Berglund F, Esguerra Merca A, Nordenskjöld B, Rutqvist LE, Skoog L, Stål O: Cytoplasmic p21WAF1/CIP1 correlates with Akt activation and poor response to tamoxifen in breast cancer. Int J Oncol 2006, 28(5):1031-1042.

216. Perez-Tenorio G, Stål O: Activation of AKT/PKB in breast cancer predicts a worse outcome among endocrine treated patients. Br J Cancer 2002, 86(4):540-545.

217. Stål O, Perez-Tenorio G, Åkerberg L, Olsson B, Nordenskjöld B, Skoog L, Rutqvist LE: Akt kinases in breast cancer and the results of adjuvant therapy. Breast Cancer Res 2003, 5(2):R37-44.

218. Khasraw M, Harvey S, Bell R: Hormonal Resistance in Breast Cancer: Evolving Treatment Strategies. Curr Breast Camcer Rep 2012, 4:66-74.

219. Harrington LS, Findlay GM, Gray A, Tolkacheva T, Wigfield S, Rebholz H, Barnett J, Leslie NR, Cheng S, Shepherd PR et al: The TSC1-2 tumor suppressor controls insulin-PI3K signaling via regulation of IRS proteins. $J$ Cell Biol 2004, 166(2):213-223.

220. Tremblay F, Brule S, Hee Um S, Li Y, Masuda K, Roden M, Sun XJ, Krebs M, Polakiewicz $\mathrm{RD}$, Thomas $\mathrm{G}$ et al: Identification of IRS-1 Ser-1101 as a target of S6K1 in nutrient- and obesity-induced insulin resistance. Proc Natl Acad Sci U S A 2007, 104(35):14056-14061.

221. Liu JL, Mao Z, LaFortune TA, Alonso MM, Gallick GE, Fueyo J, Yung WK: Cell cycledependent nuclear export of phosphatase and tensin homologue tumor suppressor is regulated by the phosphoinositide-3-kinase signaling cascade. Cancer Res 2007, 67(22):11054-11063.

222. Sridharan S, Basu A: S6 kinase 2 promotes breast cancer cell survival via Akt. Cancer Res 2011, 71(7):2590-2599.

223. Laplante M, Sabatini DM: mTOR signaling in growth control and disease. Cell 2012, 149(2):274-293.

224. Nawroth R, Stellwagen F, Schulz WA, Stoehr R, Hartmann A, Krause BJ, Gschwend JE, Retz $\mathrm{M}$ : S6K1 and 4E-BP1 are independent regulated and control cellular growth in bladder cancer. PLoS One 2011, 6(11):e27509.

225. Zhang Y, Zheng XF: mTOR-independent 4E-BP1 phosphorylation is associated with cancer resistance to mTOR kinase inhibitors. Cell cycle 2012, 11(3):594-603.

226. LeLorier J, Gregoire G, Benhaddad A, Lapierre J, Derderian F: Discrepancies between meta-analyses and subsequent large randomized, controlled trials. N Engl J Med 1997, 337(8):536-542.

227. Suresh K: An overview of randomization techniques: An unbiased assessment of outcome in clinical research. Journal of human reproductive sciences 2011, 4(1):8-11.

228. Rutqvist LE, Johansson H: Long-term follow-up of the Stockholm randomized trials of postoperative radiation therapy versus adjuvant chemotherapy among 'high risk' preand postmenopausal breast cancer patients. Acta oncologica 2006, 45(5):517-527.

229. Rutqvist LE, Johansson H, Stockholm Breast Cancer Study G: Long-term follow-up of the randomized Stockholm trial on adjuvant tamoxifen among postmenopausal patients with early stage breast cancer. Acta oncologica 2007, 46(2):133-145.

230. Jerevall PL, Ma XJ, Li H, Salunga R, Kesty NC, Erlander MG, Sgroi DC, Holmlund B, Skoog L, Fornander T et al: Prognostic utility of HOXB13:IL17BR and molecular grade index in early-stage breast cancer patients from the Stockholm trial. Br J Cancer 2011, 104(11):1762-1769.

231. Khoshnoud MR, Löfdahl B, Fohlin H, Fornander T, Stål O, Skoog L, Bergh J, Nordenskjöld $B$ : Immunohistochemistry compared to cytosol assays for determination of estrogen receptor and prediction of the long-term effect of adjuvant tamoxifen. Breast Cancer Res Treat 2011, 126(2):421-430. 
232. Stål O, Sullivan S, Sun XF, Wingren S, Nordenskjöld B: Simultaneous analysis of c-erbB-2 expression and DNA content in breast cancer using flow cytometry. Cytometry 1994, 16(2):160-168.

233. Gunnarsson C, Ahnström M, Kirschner K, Olsson B, Nordenskjöld B, Rutqvist LE, Skoog L, Stål O: Amplification of HSD17B1 and ERBB2 in primary breast cancer. Oncogene 2003, 22(1):34-40.

234. Jansson A, Delander L, Gunnarsson C, Fornander T, Skoog L, Nordenskjöld B, Stål O: Ratio of 17HSD1 to 17HSD2 protein expression predicts the outcome of tamoxifen treatment in postmenopausal breast cancer patients. Clinical cancer research : an official journal of the American Association for Cancer Research 2009, 15(10):3610-3616.

235. Stål O, Sullivan S, Wingren S, Skoog L, Rutqvist LE, Carstensen JM, Nordenskjöld B: cerbB-2 expression and benefit from adjuvant chemotherapy and radiotherapy of breast cancer. European journal of cancer 1995, 31A(13-14):2185-2190.

236. Organization ATCCSD: Cell line misidentification: the beginning of the end. Nat Rev Cancer 2010, 10.

237. Lucey BP, Nelson-Rees WA, Hutchins GM: Henrietta Lacks, HeLa cells, and cell culture contamination. Archives of pathology \& laboratory medicine 2009, 133(9):1463-1467.

238. Fire A, Xu S, Montgomery MK, Kostas SA, Driver SE, Mello CC: Potent and specific genetic interference by double-stranded RNA in Caenorhabditis elegans. Nature 1998, 391(6669):806-811.

239. Whitehead KA, Langer R, Anderson DG: Knocking down barriers: advances in siRNA delivery. Nature reviews Drug discovery 2009, 8(2):129-138.

240. Barretina J, Caponigro G, Stransky N, Venkatesan K, Margolin AA, Kim S, Wilson CJ, Lehar J, Kryukov GV, Sonkin D et al: The Cancer Cell Line Encyclopedia enables predictive modelling of anticancer drug sensitivity. Nature 2012, 483(7391):603-607.

241. Sauter G, Simon R, Hillan K: Tissue microarrays in drug discovery. Nature reviews Drug discovery 2003, 2(12):962-972.

242. Simon R, Mirlacher M, Sauter G: Tissue microarrays in cancer diagnosis. Expert review of molecular diagnostics 2003, 3(4):421-430.

243. Tan SC, Yiap BC: DNA, RNA, and protein extraction: the past and the present. Journal of biomedicine \& biotechnology 2009, 2009:574398.

244. Beer B: Bestimmung der Absorption des rothen Lichts in farbigen Flüssigkeiten" (Determination of the absorption of red light in colored liquids). Annalen der Physik und Chemie 1852, 86:78-88.

245. Lambert JH: Photometria sive de mensura et gradibus luminis, colorum et umbrae [Photometry, or, On the measure and gradations of light, colors, and shade]. Augsburg ("Augusta Vindelicorum"), Germany: Eberhardt Klet 1760.

246. Schroeder A, Mueller O, Stocker S, Salowsky R, Leiber M, Gassmann M, Lightfoot S, Menzel W, Granzow M, Ragg T: The RIN: an RNA integrity number for assigning integrity values to RNA measurements. BMC molecular biology 2006, 7:3.

247. Mullis K, Faloona F, Scharf S, Saiki R, Horn G, Erlich H: Specific enzymatic amplification of DNA in vitro: the polymerase chain reaction. Cold Spring Harbor symposia on quantitative biology 1986, 51 Pt 1:263-273.

248. Coons $\mathrm{AH}$, Creech $\mathrm{H}$, Jones R: Immunological properties of an antibody containing a fluorescent group. Proc Soc Exp Biol Med 1941, 47:200-202.

249. Bostner J: The Akt/mTOR Pathway and Estrogen Receptor Phosphorylations -a crosstalk with potential to predict tamoxifen resistance in breast cancer. Linköping University Medical Dissertations No 13792013.

250. Southern EM: Detection of specific sequences among DNA fragments separated by gel electrophoresis. Journal of molecular biology 1975, 98(3):503-517.

251. Towbin H, Staehelin T, Gordon J: Electrophoretic transfer of proteins from polyacrylamide gels to nitrocellulose sheets: procedure and some applications. Proc Natl Acad Sci U S A 1979, 76(9):4350-4354. 
252. Matos LL, Trufelli DC, de Matos MG, da Silva Pinhal MA: Immunohistochemistry as an important tool in biomarkers detection and clinical practice. Biomarker insights 2010, 5:9-20.

253. Lander ES, Linton LM, Birren B, Nusbaum C, Zody MC, Baldwin J, Devon K, Dewar K, Doyle $\mathrm{M}$, FitzHugh $\mathrm{W}$ et al: Initial sequencing and analysis of the human genome. Nature 2001, 409(6822):860-921.

254. Fodor SP, Read JL, Pirrung MC, Stryer L, Lu AT, Solas D: Light-directed, spatially addressable parallel chemical synthesis. Science 1991, 251(4995):767-773.

255. Dalma-Weiszhausz DD, Warrington J, Tanimoto EY, Miyada CG: The affymetrix GeneChip platform: an overview. Methods in enzymology 2006, 410:3-28.

256. Hicks J, Krasnitz A, Lakshmi B, Navin NE, Riggs M, Leibu E, Esposito D, Alexander J, Troge J, Grubor $\mathrm{V}$ et al: Novel patterns of genome rearrangement and their association with survival in breast cancer. Genome research 2006, 16(12):1465-1479.

257. Buckley PG, Mantripragada KK, Piotrowski A, Diaz de Stahl T, Dumanski JP: Copynumber polymorphisms: mining the tip of an iceberg. Trends in genetics : TIG 2005, 21(6):315-317.

258. Redon R, Ishikawa S, Fitch KR, Feuk L, Perry GH, Andrews TD, Fiegler H, Shapero MH, Carson $\mathrm{AR}$, Chen $\mathrm{W}$ et al: Global variation in copy number in the human genome. Nature 2006, 444(7118):444-454.

259. Lindblad-Toh K, Tanenbaum DM, Daly MJ, Winchester E, Lui WO, Villapakkam A, Stanton SE, Larsson C, Hudson TJ, Johnson BE et al: Loss-of-heterozygosity analysis of small-cell lung carcinomas using single-nucleotide polymorphism arrays. Nature biotechnology 2000, 18(9):1001-1005.

260. Teufel A, Krupp M, Weinmann A, Galle PR: Current bioinformatics tools in genomic biomedical research (Review). International journal of molecular medicine 2006, 17(6):967-973.

261. Gene Ontology C: Creating the gene ontology resource: design and implementation. Genome research 2001, 11(8):1425-1433.

262. Reimand J, Arak T, Vilo J: g:Profiler--a web server for functional interpretation of gene lists (2011 update). Nucleic acids research 2011, 39(Web Server issue):W307-315.

263. Reimand J, Kull M, Peterson H, Hansen J, Vilo J: g:Profiler--a web-based toolset for functional profiling of gene lists from large-scale experiments. Nucleic acids research 2007, 35(Web Server issue):W193-200.

264. Blundell TL, Sibanda BL, Sternberg MJ, Thornton JM: Knowledge-based prediction of protein structures and the design of novel molecules. Nature 1987, 326(6111):347-352.

265. Dill KA, MacCallum JL: The protein-folding problem, 50 years on. Science 2012, 338(6110):1042-1046.

266. Marti-Renom MA, Stuart AC, Fiser A, Sanchez R, Melo F, Sali A: Comparative protein structure modeling of genes and genomes. Annual review of biophysics and biomolecular structure 2000, 29:291-325.

267. Maiti R, Van Domselaar GH, Zhang H, Wishart DS: SuperPose: a simple server for sophisticated structural superposition. Nucleic acids research 2004, 32(Web Server issue):W590-594.

268. Barrett T, Troup DB, Wilhite SE, Ledoux P, Evangelista C, Kim IF, Tomashevsky M, Marshall KA, Phillippy KH, Sherman PM et al: NCBI GEO: archive for functional genomics data sets--10 years on. Nucleic acids research 2011, 39(Database issue):D10051010.

269. Rustici G, Kolesnikov N, Brandizi M, Burdett T, Dylag M, Emam I, Farne A, Hastings E, Ison J, Keays $\mathrm{M}$ et al: ArrayExpress update--trends in database growth and links to data analysis tools. Nucleic acids research 2013, 41(Database issue):D987-990.

270. Brazma A, Parkinson H, Sarkans U, Shojatalab M, Vilo J, Abeygunawardena N, Holloway E, Kapushesky M, Kemmeren P, Lara GG et al: ArrayExpress--a public repository for microarray gene expression data at the EBI. Nucleic acids research 2003, 31(1):68-71. 
271. Miller LD, Smeds J, George J, Vega VB, Vergara L, Ploner A, Pawitan Y, Hall P, Klaar S, Liu ET et al: An expression signature for p53 status in human breast cancer predicts mutation status, transcriptional effects, and patient survival. Proc Natl Acad Sci U S A 2005, 102(38):13550-13555.

272. Pawitan Y, Bjohle J, Amler L, Borg AL, Egyhazi S, Hall P, Han X, Holmberg L, Huang F, Klaar $\mathrm{S}$ et al: Gene expression profiling spares early breast cancer patients from adjuvant therapy: derived and validated in two population-based cohorts. Breast Cancer Res 2005, 7(6):R953-964.

273. van de Vijver MJ, He YD, van't Veer LJ, Dai H, Hart AA, Voskuil DW, Schreiber GJ, Peterse $\mathrm{JL}$, Roberts $\mathrm{C}$, Marton $\mathrm{MJ}$ et al: A gene-expression signature as a predictor of survival in breast cancer. $N$ Engl J Med 2002, 347(25):1999-2009.

274. Fisher RA: On the interpretation of $\chi \mathbf{2}$ from contingency tables, and the calculation of $\mathbf{P}$. Journal of the Royal Statistical Society 1922, 85(1):87-94.

275. Spearman C: The proof and measurement of association between two things. Amer $J$ Psychol 1904, 15:72-101.

276. Kaplan M, P M: Non-parametric estimation from incomplete observations. Journal of the American Statistical association 1958, 53:457-481.

277. Mantel N: Evaluation of survival data and two new rank order statistics arising in its consideration. Cancer chemotherapy reports Part 1 1966, 50(3):163-170.

278. Cox DR: Regression models and life tables. J R Stat Soc B 1972, 34:187-220

279. Beroukhim R, Getz G, Nghiemphu L, Barretina J, Hsueh T, Linhart D, Vivanco I, Lee JC, Huang $\mathrm{JH}$, Alexander S et al: Assessing the significance of chromosomal aberrations in cancer: methodology and application to glioma. Proc Natl Acad Sci U S A 2007, 104(50):20007-20012.

280. Irizarry RA, Hobbs B, Collin F, Beazer-Barclay YD, Antonellis KJ, Scherf U, Speed TP: Exploration, normalization, and summaries of high density oligonucleotide array probe level data. Biostatistics 2003, 4(2):249-264.

281. Barlund M, Forozan F, Kononen J, Bubendorf L, Chen Y, Bittner ML, Torhorst J, Haas P, Bucher C, Sauter G et al: Detecting activation of ribosomal protein S6 kinase by complementary DNA and tissue microarray analysis. Journal of the National Cancer Institute 2000, 92(15):1252-1259.

282. Filonenko VV, Tytarenko R, Azatjan SK, Savinska LO, Gaydar YA, Gout IT, Usenko VS, Lyzogubov VV: Immunohistochemical analysis of S6K1 and S6K2 localization in human breast tumors. Exp Oncol 2004, 26(4):294-299.

283. Yoshida S, Matsumoto K, Arao T, Taniguchi H, Goto I, Hanafusa T, Nishio K, Yamada Y: Gene amplification of ribosomal protein $\mathbf{S 6}$ kinase-1 and -2 in gastric cancer. Anticancer research 2013, 33(2):469-475.

284. Gual P, Le Marchand-Brustel Y, Tanti JF: Positive and negative regulation of insulin signaling through IRS-1 phosphorylation. Biochimie 2005, 87(1):99-109.

285. Hao Y, Wang C, Cao B, Hirsch BM, Song J, Markowitz SD, Ewing RM, Sedwick D, Liu L, Zheng W et al: Gain of interaction with IRS1 by p110alpha-helical domain mutants is crucial for their oncogenic functions. Cancer Cell 2013, 23(5):583-593.

286. Schiff R, Massarweh SA, Shou J, Bharwani L, Mohsin SK, Osborne CK: Cross-talk between estrogen receptor and growth factor pathways as a molecular target for overcoming endocrine resistance. Clinical cancer research : an official journal of the American Association for Cancer Research 2004, 10 (1 Pt 2):331S-336S.

287. Bekri S, Adelaide J, Merscher S, Grosgeorge J, Caroli-Bosc F, Perucca-Lostanlen D, Kelley PM, Pebusque MJ, Theillet C, Birnbaum D et al: Detailed map of a region commonly amplified at 11q13-->q14 in human breast carcinoma. Cytogenetics and cell genetics 1997, 79(1-2):125-131.

288. Hui R, Campbell DH, Lee CS, McCaul K, Horsfall DJ, Musgrove EA, Daly RJ, Seshadri R, Sutherland RL: EMS1 amplification can occur independently of CCND1 or INT-2 
amplification at 11q13 and may identify different phenotypes in primary breast cancer. Oncogene 1997, 15(13):1617-1623.

289. Rodriguez C, Hughes-Davies L, Valles H, Orsetti B, Cuny M, Ursule L, Kouzarides T, Theillet C: Amplification of the BRCA2 pathway gene EMSY in sporadic breast cancer is related to negative outcome. Clinical cancer research : an official journal of the American Association for Cancer Research 2004, 10(17):5785-5791.

290. Kwek SS, Roy R, Zhou H, Climent J, Martinez-Climent JA, Fridlyand J, Albertson DG: Coamplified genes at 8p12 and 11q13 in breast tumors cooperate with two major pathways in oncogenesis. Oncogene 2009, 28(17):1892-1903.

291. Fu M, Wang C, Li Z, Sakamaki T, Pestell RG: Minireview: Cyclin D1: normal and abnormal functions. Endocrinology 2004, 145(12):5439-5447.

292. Doisneau-Sixou SF, Sergio CM, Carroll JS, Hui R, Musgrove EA, Sutherland RL: Estrogen and antiestrogen regulation of cell cycle progression in breast cancer cells. Endocrinerelated cancer 2003, 10(2):179-186.

293. Turner N, Pearson A, Sharpe R, Lambros M, Geyer F, Lopez-Garcia MA, Natrajan R, Marchio C, Iorns E, Mackay A et al: FGFR1 amplification drives endocrine therapy resistance and is a therapeutic target in breast cancer. Cancer Res 2010, 70(5):2085-2094.

294. Benavente S, Verges R, Hermosilla E, Fumanal V, Casanova N, Garcia A, Ramon YCS, Giralt J: Overexpression of phosphorylated 4E-BP1 predicts for tumor recurrence and reduced survival in cervical carcinoma treated with postoperative radiotherapy. International journal of radiation oncology, biology, physics 2009, 75(5):1316-1322.

295. O'Reilly KE, Warycha M, Davies MA, Rodrik V, Zhou XK, Yee H, Polsky D, Pavlick AC, Rosen N, Bhardwaj $\mathrm{N}$ et al: Phosphorylated 4E-BP1 is associated with poor survival in melanoma. Clinical cancer research : an official journal of the American Association for Cancer Research 2009, 15(8):2872-2878.

296. Michalopoulos I, Pavlopoulos GA, Malatras A, Karelas A, Kostadima MA, Schneider R, Kossida S: Human gene correlation analysis (HGCA): a tool for the identification of transcriptionally co-expressed genes. BMC research notes 2012, 5:265.

297. Reynier F, Petit F, Paye M, Turrel-Davin F, Imbert PE, Hot A, Mougin B, Miossec P: Importance of correlation between gene expression levels: application to the type I interferon signature in rheumatoid arthritis. PLoS One 2011, 6(10):e24828.

298. Bosco EE, Knudsen ES: RB in breast cancer: at the crossroads of tumorigenesis and treatment. Cell cycle 2007, 6(6):667-671.

299. Biswas AK, Johnson DG: Transcriptional and nontranscriptional functions of E2F1 in response to DNA damage. Cancer Res 2012, 72(1):13-17.

300. Chen HZ, Tsai SY, Leone G: Emerging roles of E2Fs in cancer: an exit from cell cycle control. Nat Rev Cancer 2009, 9(11):785-797.

301. Engelmann D, Putzer BM: The dark side of E2F1: in transit beyond apoptosis. Cancer Res 2012, 72(3):571-575.

302. Vuaroqueaux V, Urban P, Labuhn M, Delorenzi M, Wirapati P, Benz CC, Flury R, Dieterich $\mathrm{H}$, Spyratos F, Eppenberger U et al: Low E2F1 transcript levels are a strong determinant of favorable breast cancer outcome. Breast Cancer Res 2007, 9(3):R33.

303. Real S, Meo-Evoli N, Espada L, Tauler A: E2F1 regulates cellular growth by mTORC1 signaling. PLoS One 2011, 6(1):e16163.

304. Pollak M: The insulin and insulin-like growth factor receptor family in neoplasia: an update. Nat Rev Cancer 2012, 12(3):159-169.

305. Deblois G, Giguere V: Oestrogen-related receptors in breast cancer: control of cellular metabolism and beyond. Nat Rev Cancer 2013, 13(1):27-36.

306. Ochnik AM, Yee D: Estrogen-related receptor alpha: an orphan finds a family. Breast Cancer Res 2012, 14(3):309.

307. Vogel C, Marcotte EM: Insights into the regulation of protein abundance from proteomic and transcriptomic analyses. Nature reviews Genetics 2012, 13(4):227-232.

308. Meric-Bernstam F, Chen H, Akcakanat A, Do KA, Lluch A, Hennessy BT, Hortobagyi GN, Mills GB, Gonzalez-Angulo AM: Aberrations in translational regulation are associated 
with poor prognosis in hormone receptor-positive breast cancer. Breast Cancer Res 2012, 14(5):R138.

309. Wood CE, Register TC, Cline JM: Transcriptional profiles of progestogen effects in the postmenopausal breast. Breast Cancer Res Treat 2009, 114(2):233-242.

310. Beelen $\mathrm{K}$, Zwart W, Linn SC: Can predictive biomarkers in breast cancer guide adjuvant endocrine therapy? Nature reviews Clinical oncology 2012, 9(9):529-541.

311. Droog M, Beelen K, Linn S, Zwart W: Tamoxifen resistance: From bench to bedside. European journal of pharmacology 2013, 717(1-3):47-57.

312. Harrison C: Kinase inhibitors: Analysing kinase inhibitor selectivity. Nature reviews Drug discovery 2012, 11(12). 


\section{Original Publications}

The articles associated with this thesis have been removed for copyright reasons. For more details about these see:

http://urn.kb.se/resolve?urn=urn:nbn:se:liu:diva-104180 